\title{
Numerical modelling of microscopic lubricant flow in sheet metal forming. Application to plane strip drawing
}

Carretta, Y.; Boman, R.; Bech, Jakob Ilsted; Legrand, N.; Laugier, M.; Ponthot, J.-P.

Published in:

International Journal for Numerical Methods in Engineering

Link to article, DOI:

$10.1002 / \mathrm{nme} .5509$

Publication date:

2017

Document Version

Peer reviewed version

Link back to DTU Orbit

Citation (APA):

Carretta, Y., Boman, R., Bech, J. I., Legrand, N., Laugier, M., \& Ponthot, J-P. (2017). Numerical modelling of microscopic lubricant flow in sheet metal forming. Application to plane strip drawing. International Journal for Numerical Methods in Engineering, 112(3), 203-237. https://doi.org/10.1002/nme.5509

\section{General rights}

Copyright and moral rights for the publications made accessible in the public portal are retained by the authors and/or other copyright owners and it is a condition of accessing publications that users recognise and abide by the legal requirements associated with these rights.

- Users may download and print one copy of any publication from the public portal for the purpose of private study or research.

- You may not further distribute the material or use it for any profit-making activity or commercial gain

- You may freely distribute the URL identifying the publication in the public portal 


\title{
Numerical modelling of microscopic lubricant flow in sheet metal forming. Application to plane strip drawing.
}

\author{
Y Carretta ${ }^{1}$, R Boman ${ }^{1}$, J Bech ${ }^{2}$, N Legrand ${ }^{3}$, M Laugier ${ }^{3}$, J-P Ponthot ${ }^{1}$ \\ ${ }^{1}$ Department of Aerospace and Mechanical Engineering, University of Liège, Belgium \\ y.carretta@ulg.ac.be \\ r.boman@ulg.ac.be \\ jp.ponthot@ulg.ac.be \\ ${ }^{2}$ Department of Wind Energy, Technical University of Denmark, 2800 Lyngby, Denmark \\ jakb@dtu.dk
}

${ }^{3}$ ArcelorMittal Global R\&D, Maizières-les-Metz, France nicolas.legrand@arcelormittal.com

maxime.laugier@arcelormittal.com

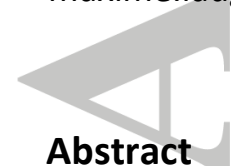

This paper presents a numerical investigation of microscopic lubricant flows from the cavities to the plateaus of the surface roughness of metal sheets during forming processes. This phenomenon, called micro-plasto-hydrodynamic (MPH) lubrication, was observed experimentally in various situations such as compression sliding tests, strip drawing and cold rolling. It leads to local friction drop and wear reduction. It is therefore critical to achieve a good understanding of this phenomenon.

To move towards that goal, a multiscale fluid-structure interaction (FSI) model is developed to model lubricant flows at the microscopic scale. These simulations are made possible through the use of the Arbitrary Lagrangian Eulerian (ALE) formalism.

In this paper, this methodology is used to study plane strip drawing. The numerical model is able to predict the onset of lubricant escape and the amount of lubricant flowing on the plateaus. Numerical results exhibit good agreement with experimental measurements.

\section{Keywords.}

Metal forming; micro-plasto-hydrodynamic (MPH) lubrication; Finite element method; Liquid lubrication mechanisms; ALE formulation

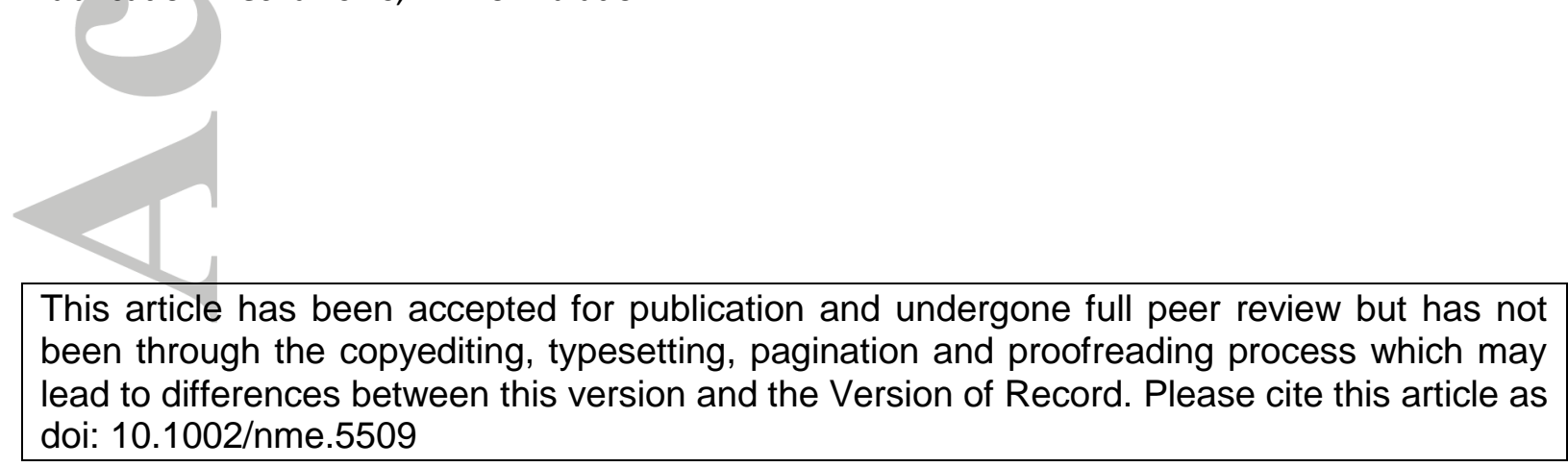

This article is protected by copyright. All rights reserved. 


\section{Introduction}

This paper presents a new numerical model capable of modelling lubricant escapes from microscopic cavities in plane strip drawing. Such lubricant flows were observed experimentally in different processes such as compression friction tests [1] plane strip drawing $[2,3,4,5,6]$ and cold rolling [7, 8]. Despite these evidences and their effect on local friction conditions, the phenomenon has not been addressed successfully in any numerical simulations.

Currently, for most metal forming simulations, the tool and material surfaces are geometrically modelled as smooth macroscopic surfaces and the friction forces are modelled through rather simplistic friction laws such as Coulomb's or Tresca's. This is not enough to predict accurately the forces or the contact pressure profiles when the operating parameters of the process are changed. Indeed, the friction coefficient value has to be adjusted as a function of local (microscopic) conditions to match experimental measurements. This is not surprising since the phenomena taking place at the microscopic level are too complex to be represented by a single friction coefficient value.

At the microscopic level, the surface of the tool and the deformable part exhibits asperities and valleys. When metal parts are in contact with the forming tools, contact occurs at asperity peaks. Therefore, the real contact area is lower than the apparent (nominal) contact area, the pressure at asperity tops is large and local plastic deformations known as asperity flattening take place. When a relative sliding motion between the material and the tool happens, ploughing [9] comes into play: asperities encounter each other which contributes to friction forces.

When very clean metal surfaces come into contact, adhesion - due to interatomic forces - happens. Adhesion is avoided when a contaminant such as an oxide layer covers the asperities. Metal or polymer films as well as lubricants with oiliness and extreme pressure additives can also be used to protect the contacting surfaces in order to reduce adhesion.

The effect of the three main sources of friction mentioned above - asperity flattening, ploughing and adhesion - can be reduced through the use of lubricants : adhesion is prevented through chemical additives (such as ester in cold rolling) whereas asperity flattening and ploughing are brought down when the contacting surfaces are separated by a lubricant layer. Different lubricant regimes take place depending on the operating conditions and the piezo viscous behaviour of the lubricant itself.

When the two surfaces are fully separated by the lubricant film and thick-film lubrication occurs. When the lubricant film thickness decreases to a value of the same order of magnitude as the surfaces roughness, thin-film regime occurs and asperities have an effect on the lubricant flow. Once the film thickness decreases even further, mixed lubrication regime takes place. There, both solid-tosolid and fluid-to-solid contact conditions happen and friction is the mixture of these two components. Finally, when the contact load is only supported by asperities of the contacting surfaces, the boundary lubrication regime is reached.

Except in the boundary regime, friction conditions depend on a fluid component. In thick-film lubrication conditions, the fluid pressure can be computed using Reynolds' equation [10]. Patir and Cheng [11] added flow factors to that equation to account for surface roughness in the thin-film regime. This modified equation has been used to model sheet metal forming processes accounting for the effect of asperity interactions. Hu and Liu [12] were the first to implement it into a finite 
element (FE) code to model strip rolling in the thin-film regime. Then, Boman and Ponthot [13] extended Hu and Liu's formulation to make it independent from the process parameters. Yang and Lo [14] also used Patir and Cheng's equation to deal with stretch forming processes.

To carry out numerical simulations in the mixed lubrication regime, Wilson and Marsault [15] extended Patir and Cheng's work to deal with large relative contact areas. In that case, the solid contribution to friction has to be taken into account through an asperity-crushing model. Wilson and Sheu [16] and Sutcliffe [17] provided semi-empirical relationships linking the relative contact area to the hardness of the material and plastic deformation of the bulk.

Sheu and Wilson [18], Marsault [19], Qiu et al. [20] and then Stephany [21 - 22] coupled Wilson and Sheu's [16] semi-empirical law for asperity crushing to Wilson and Marsault [15] equation to compute shear stress distribution in cold-rolling. Also for the mixed lubrication regime, Hol et al. [23] developed a multi-scale approach to determine local friction coefficient distribution in strip drawing based on Westeneng's [24] asperity crushing model.

Even though the aforementioned models are becoming more and more realistic, none of them deals properly with configurations where the lubricant is completely trapped in isolated surface pockets. Experimental observation suggests that oil trapped in these cavities can behave as a source of lubricant to reduce friction. Mizuno and Okamoto [1] observed this phenomenon in compression friction tests. Strip surface analysis performed after the experiments showed discrete oil pits at low speed and small viscosity. At higher sliding speed and viscosity, these pits decreased in size meaning that the lubricant flew out of the cavities. Their experimental results also showed an increase of the mean shear stress with the product of the sliding velocity and the viscosity of the oil. This means that some hydrodynamic phenomenon took place at the interface. They termed this phenomenon "micro-plasto-hydrodynamic" (MPH) lubrication.

Azushima et al. [Azushima1990], Bech et al. [4], Sørensen et al. [5], and Shimizu et al. [6] used a test rig to study these lubricant flows in strip drawing. Through a tool made of glass they observed the behaviour of macroscopic pyramidal indentations filled with lubricant. While the strip was pulled through the die, they witnessed lubricant being squeezed out from the oil pockets at the front and/or at the rear of the pyramidal cavities depending on operational parameters. They showed lubricant escapes were influenced by various process parameters such as strip thickness reduction, drawing speed, lubricant viscosity, die angle, friction conditions along the lower die, etc.

Azushima [3] used the same experimental device to achieve direct observation at the asperity level through a microscope. He observed microroughening of the plateaus caused by lubricant drawn out of the pits. Ahmed and Sutcliffe [7] conducted strip drawing and rolling experiments highlighting the occurrence of MPH lubrication in these two processes at the microscopic scale. Aslo, Laugier et al. [8] computed equivalent friction coefficient values based on process experimental measurements (rolling load and forward slip) for different strip thickness reduction levels. They observed a decrease of these friction coefficient values with the strip thickness reduction suggesting the occurrence of $\mathrm{MPH}$ lubrication.

There exist only a few models dealing with MPH. Lo and Wilson [25] developed equations to model lubricant outflows at the rear of lubricant cavities. Even though this model represents some features of MPH lubrication, it suffers from several limitations (rigid-plastic material, constant slope of the 
cavity edge during the deformation, etc.). These equations were then extended by Sutcliffe et al. [26] to investigate evolution of micro-pits during strip rolling and drawing due to MPH lubrication.

Shimizu et al. [27] used a rigid-viscoplastic finite element formulation to model plane strip drawing experiments. A two-step procedure was developed. In the first step, velocities of the nodes on the edges of the macroscopic lubricant cavity were recorded assuming the material of the strip and the cavity were identical. Then, during the second step, these boundary conditions were applied on the edges of a cavity filled with lubricant. Therefore, fluid and solid parts were not accounted for at the same time during the simulation.

Dubois et al. [28 - 29] used a fluid-solid coupling approach implemented in a finite element simulation code to model lubricant exchanges between consecutive pockets in plane strip drawing. The model limitation is the direct connexion between two consecutive cavities from the beginning of the simulations. This does not allow them to determine the onset of the MPH flows. Moreover, the thickness of the link is not a result of the simulation but it has to be defined a priori and cannot evolve during the simulation.

This paper presents a fluid-structure interaction (FSI) model capable of predicting the onset of MPH lubrication and the amount of lubricant escape. This method was developed in Metafor [30], an inhouse FE code including large strains, and then used to reproduce experiments conducted by Bech et al. [4] in plane strip drawing.

Since Metafor was initially developed to perform metal forming simulations, the formalism used in the program is therefore suitable for solids. Results discussed in section 2 show that low Reynolds flow simulations with viscous fluid can be carried out with this solid code. The velocity field and the pressure field predictions are respectively validated thanks to the lid-driven cavity, a well-known test case in fluid mechanics, and the plane slide pad bearing test. These fluid simulations were made possible through the use of the Arbitrary Lagrangian Eulerian (ALE) formalism which allows us to uncouple the motion of the mesh and the material. The main features of the ALE method are also described in section 2 .

A FSI benchmark is also discussed in section 1. This test case consists of a flexible beam located ahead of a converging channel. The displacement of the beam as well as the pressure in the fluid are in good agreement with two numerical results from the literature [31-32] and therefore show the ability of the FE code to deal with FSI simulations.

The multi-scale MPH lubrication model is described in section 3. This original model is able to predict lubricant escapes of the pocket backward and forward and shows good agreement with experimental observations carried out by Bech et al. 


\section{Fluid flow simulations using a solid mechanics code}

\subsection{Introduction}

As mentioned in the introduction, the FE software Metafor used in this study was originally developed to model the behaviour of solids. It is employed in the present work to carry out fluidstructure interaction simulations where the fluid is assumed to be Newtonian.

Stresses in the fluid are computed with the Norton-Hoff material law in the Lagrangian formalism which is mostly used to deal with solid materials. In this formalism, the material sticks to the mesh on the contrary to the Eulerian Formalism where the mesh is fixed and the material flows through the mesh. The Eulerian formalism is mainly utilized in fluid flow simulations. The drawback of such a formulation is that it is not easy to account for moving boundaries as it is the case in fluid-structure interactions simulations. To do so, the Arbitrary Lagrangian Eulerian (ALE) formalism, which uncouples the motion of the mesh and the material, is employed. Practically speaking, the fluid is able to cross the mesh boundaries during the simulation. Therefore, fluid flows can be computed with limited mesh distortion which would be detrimental to the convergence and the quality of the results.

The following paragraphs discuss the kinematics used for large deformation continuum. Then the integration of the Norton-Hoff material law involving these kinematics is explained. Information regarding the ALE procedure are then provided.

\subsubsection{Overview of the kinematics for large deformation continuum}

The kinematical description discussed below [33] is obtained by considering two configurations of a body: the reference configuration and the current configuration. In the reference configuration, the position of a given particle, at a given time $t_{0}$, is given by its position vector $\mathbf{X}$ whereas, in the current configuration, at time $t$, the position of the same particle is given by $\mathbf{x}$. The relation between these two vectors is given by

$$
x=x(X, t)
$$

The link between these two configurations is the deformation gradient, given by the second-rank two-point tensor $\boldsymbol{F}$

$$
\boldsymbol{F}=\frac{\partial \boldsymbol{x}}{\partial \boldsymbol{X}} \text { with } J=\operatorname{det} \boldsymbol{F}>0
$$

The deformation gradient can be uniquely decomposed by the polar decomposition as

$$
\begin{gathered}
F=R U \\
\text { where } R^{T} R=I \text { and } U=U^{T}
\end{gathered}
$$


The velocity of the reference point $\mathrm{X}$ in the current configuration is given by

$$
v=\dot{x}=\frac{\partial x(X, t)}{\partial t}
$$

The corresponding spatial gradient of velocity is given by

$$
L=\frac{\partial v}{\partial x}=\dot{F} F^{-1}
$$

which, in turns, can be decomposed into a symmetric and antisymmetric part

$$
L=D+W
$$

where $\mathbf{D}$ and $\mathbf{W}$ are respectively the rate of deformation and the spin tensor

$$
\begin{aligned}
& D=\frac{1}{2}\left(L+L^{T}\right) \\
& W=\frac{1}{2}\left(L-L^{T}\right)
\end{aligned}
$$

\subsubsection{Norton-Hoff material law}

As previously said, the stress tensor in the fluid is obtained by assuming a Norton-Hoff material model. In the present case, the stress tensor is split into its deviatoric part and the hydrostatic part.

The following equation determines the cauchy stress deviator $s_{i j}$

$$
s_{i j}=2 \eta D_{i j}\left(\sqrt{3} \sqrt{\frac{2}{3} D_{l k} D_{l k}}\right)^{m-1}=2 \eta D_{i j}(\sqrt{3} \dot{\bar{\varepsilon}})^{m-1}
$$

as a function of

- $\eta$ the dynamic viscosity,

- $D_{i j}$ the strain rate tensor,

- $m$ the strain rate sensitivity coefficient,

- $\dot{\bar{\varepsilon}}$ the equivalent strain rate with $\dot{\bar{\varepsilon}}=\sqrt{\frac{3}{2} D_{l k} D_{l k}}$.

By choosing $m=1$, we obtain the equation of a Newtonian fluid:

$$
s_{i j}=2 \eta D_{i j}
$$

All these terms can be in the current configuration. Thus, no integration is required to determine the current components of the tensor $\mathbf{s}$.

This article is protected by copyright. All rights reserved. 
To compute the complete stress tensor, pressure terms are added to the deviatoric tensor. The pressure variation between the reference and the current configuration is obtained by integrating the following equation

where $\mathrm{p}$ is the pressure and $\mathrm{K}$ is the bulk modulus

$$
\dot{p}=K \operatorname{tr}(\boldsymbol{D})
$$

By integrating that equation from the beginning to the end of the time step and assuming a constant bulk modulus, we obtain, see e.g. [33]:

$$
\Delta p=K \int_{t 0}^{t 1} \operatorname{tr}(\boldsymbol{D}) d t=K \operatorname{tr}\left(E^{N}\right)
$$

where $E^{N}$ is the increment of the natural or logarithmic strain tensor over the time step.

Thus, the pressure is computed incrementally at each time step with the following equation

$$
p_{1}=p_{0}+K \operatorname{tr}\left(E^{N}\right)
$$

where $p_{0}$ and $p_{1}$ are the pressure in the elements respectively at the beginning and the end of the time step.

The mesh used over the 2D fluid domain is made of Q1P0 hybrid finite elements: four Gauss points are used to integrate the deviatoric stress deviator while the pressure is assumed constant all over the element and is computed at the element centre to prevent locking. These elements are also named SRI for Selective Reduced Integration.

Knowing the evolution of the positions of the nodes, it is possible to integrate the material laws used in the model in order to determine the stresses over the elements. Nodal forces are then computed and the force equilibrium is assessed. Nodal positions are then updated for the whole domain by a Newton-Raphson procedure. This process goes on until the force equilibrium is satisfied.

\subsubsection{Arbitrary Lagrangian Eulerian formalism}

As previously said, the fluid simulations are made possible thanks to the use of the Arbitrary Lagrangian-Eulerian (ALE). The present formulation [34-35] uses an operator splitting to integrate the equations in time and each time step is divided into two stages. The first one is Lagrangian and the material sticks to the mesh as in a classical Lagrangian simulation. The second stage is made of a node relocation followed by a convective Eulerian phase, transferring the unknowns stored at the nodes (velocities) and at the Gauss points (stress components) from the old mesh to the new one.

The convection method used here, called Godunov-type update technique, is first-order accurate and is based on a finite volume method [35].

The time integration is achieved using a Chung-Hulbert implicit dynamic scheme [36] where the parameters $\alpha_{M}, \alpha_{F}, \beta_{0}, \gamma_{0}$ are respectively set to $-0.97,0.01,0.25$ and 0.5 . 


\subsection{Fluid simulation validity}

The equations presented in the section 2.1.1 general equations from continuum mechanics and apply to both fluids and solids. The constitutive model presented in section 2.1.2 is a Norton-Hoff model (from solid mechanics) that is quite similar to a non-Newtonian model from fluid mechanics as far as deviatoric stresses are concerned.

The pressure model in equation 14.1, can simply be interpreted in the sense that the pressure is directly proportional to the logarithm of the current volume (at the end of the time step, $V$ ) divided by the reference volume (at the beginning of the time step $V_{0}$ ):

$$
\operatorname{tr}\left(E^{N}\right)=\ln \frac{V}{V_{0}}=\ln J=\ln (\operatorname{det} F)
$$

As the bulk modulus $K$, the constant of proportionality is generally quite a large number, this is a standard formulation in solid mechanics to represent a slightly compressible medium.

However the formalism in which these equations are presented here was originally developed for solids. Therefore, the present approach might not be suitable to cover all types of fluid mechanics applications: for example, studying the air flow around an aircraft wing would not be very efficient with our approach. Nonetheless, according to Zienkiewicz [45], low speed viscous flows can be solved with the solid formalism. In that sense, our approach relates to compressible Stokes flow models where advective inertial forces are small compared to viscous forces.

To ensure Zienkiewicz's statement applied in our case, several fluid flow simulations have been conducted to assess the validity of the results computed with the present approach. The following paragraphs present two of these tests. The first one is the lid-driven cavity, a well-known test case in fluid mechanics (see [37 - 38 - 39 - 40 - 41] among others). It shows the validity of the velocity field computation. The second one deals with a lubricant flow in a converging gap [42, 43]. It shows the validity of the pressure field computed in the fluid domain.

For both tests, the lubricant exhibits a Newtonian behavior with a constant dynamic viscosity $\eta=0.3$ Pa.s and a constant bulk modulus K $=1700 \mathrm{MPa}$ which are typical values for oil as presented later in this contribution.

Both meshes are Eulerian which means that the nodes are moved back to their initial position at each time step.

\subsubsection{Lid driven cavity}

The geometry and the mesh of the square cavity are shown in Figure 1 and Figure 2. A constant horizontal velocity $v_{0}=1 \mathrm{~mm} / \mathrm{s}$ is applied on the upper edge generating a vortex in the fluid. A noslip condition is applied on the other edges. This simulation is conducted both with Metafor (ALE formulation) and with OpenFOAM (finite volume code - Eulerian formulation) [44], an open source CFD software package. The icoFoam solver from OpenFOAM is used. It is suitable for laminar, isothermal and incompressible flow. 
A comparison of the steady state velocity field computed with the two numerical tools is made in Figure 3 - $a$ and $b$. The results are very similar and the streamlines (in white) have the same shape.

To avoid numerical instabilities in the FE simulation (see Figure $3-\mathrm{c}$ ), the adhesion condition is relaxed on the two vertical edges near the two upper corners (see Figure $3-b$ where four upper nodes on each edge are free to move vertically i.e. "free slip state"). This is a drawback of the present approach: fluid simulations conducted with the solid formalism are more sensitive to boundary conditions. However, the effect this modification has on the solution is limited to the neighbourhood of the two corners.

The horizontal velocity profile on the vertical line going through the centre of the geometry is represented in Figure 4. Metafor results are compared to those obtained with OpenFOAM and to a reference solution proposed by Zienkiewicz [45]. These three curves match perfectly. The same conclusion is drawn from the vertical velocity profile extracted on the horizontal line in the middle of the fluid domain (see Figure 5).

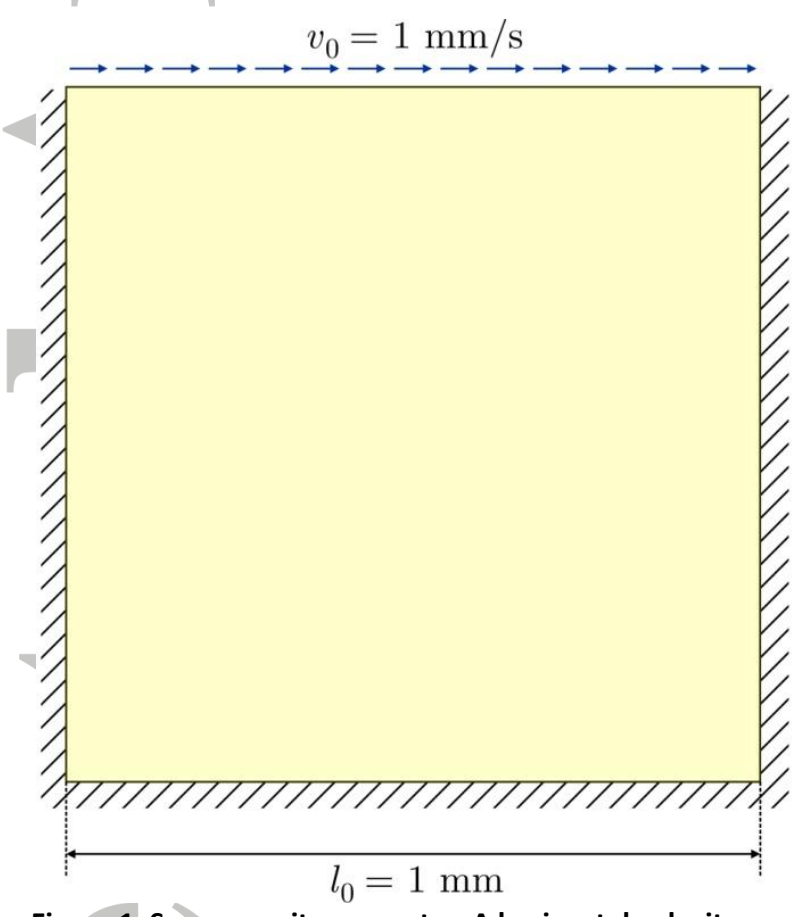

Figure 1. Square cavity geometry. A horizontal velocity $v_{0}=1 \mathrm{~mm} / \mathrm{s}$ is applied on the upper edge of the geometry.

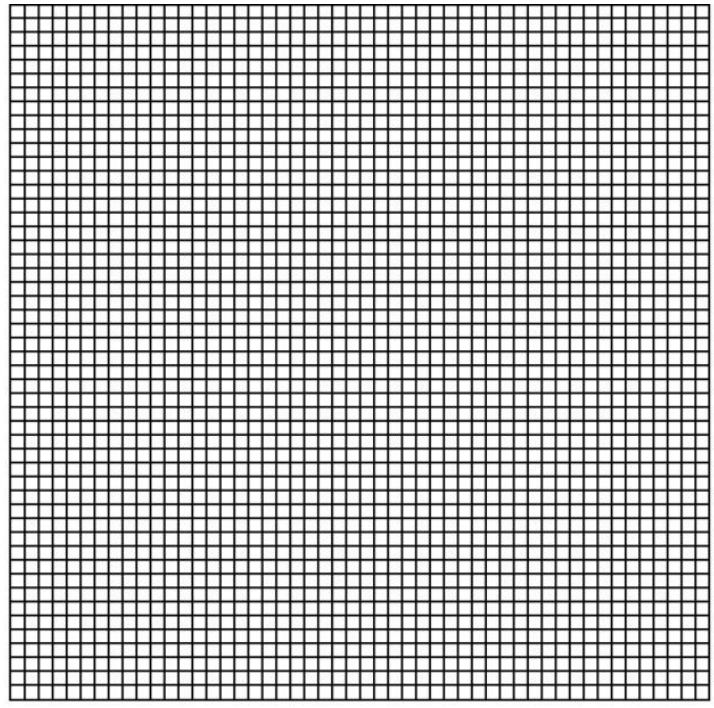

Figure 2. Mesh used in the simulation. It is made of $\mathbf{2 5 0 0}$ square elements. 
a) OpenFOAM

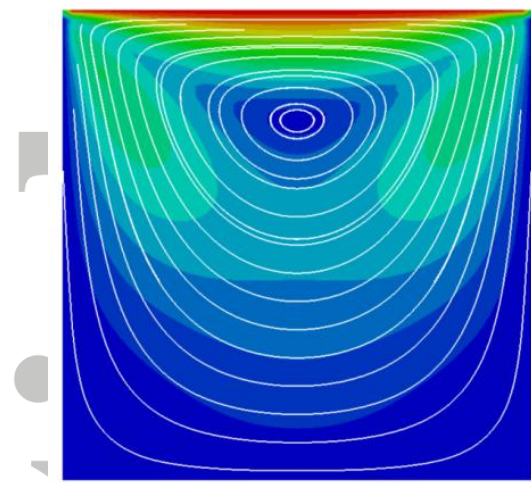

0 b) Metafor with specific BC

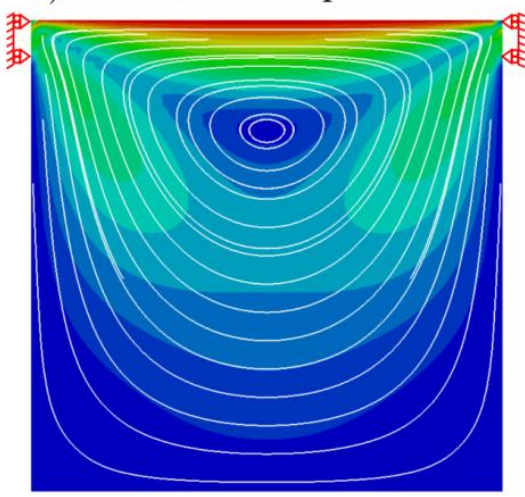

c) Metafor without specific BC

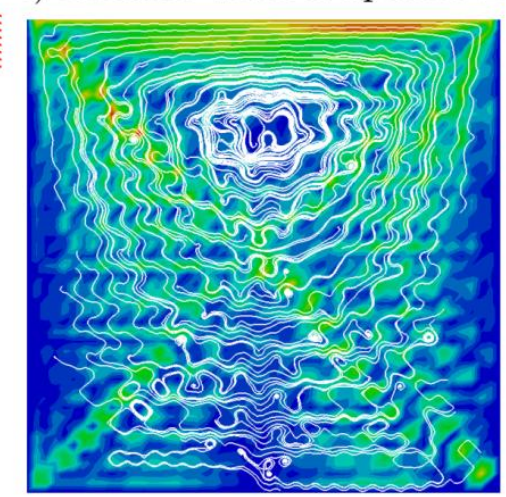

Velocity magnitude $[\mathrm{mm} / \mathrm{s}]$
0.25
0.5
0.75

1

Figure 3. a) velocity field and streamlines computed with OpenFOAM. b) numerical results obtained with the FE software Metafor when adding rolling support conditions (equivalent to free slip conditions) near the two upper corner of the cavity. c) Numerical instabilities occurring in Metafor when rolling support conditions are not used (no-slip conditions are applied all along the two edges).

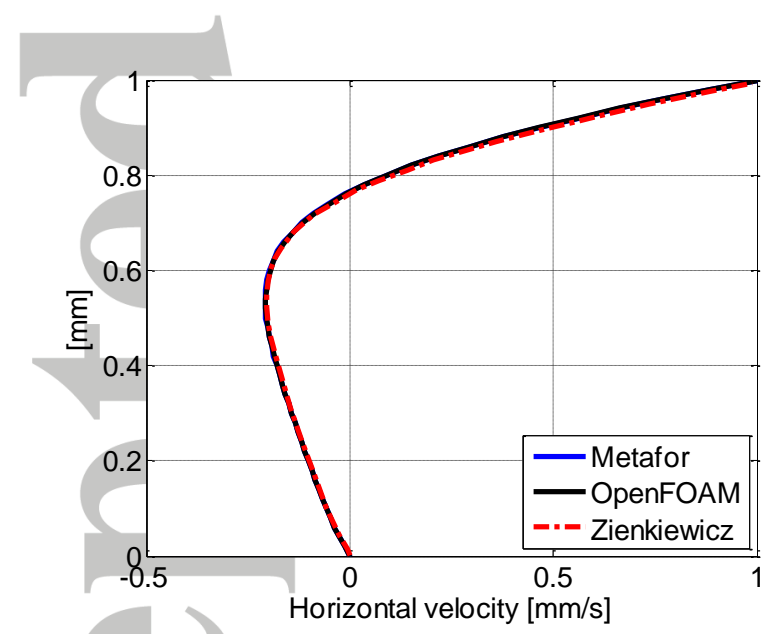

Figure 4. Horizontal velocity profile on the vertical line in the middle of the geometry.

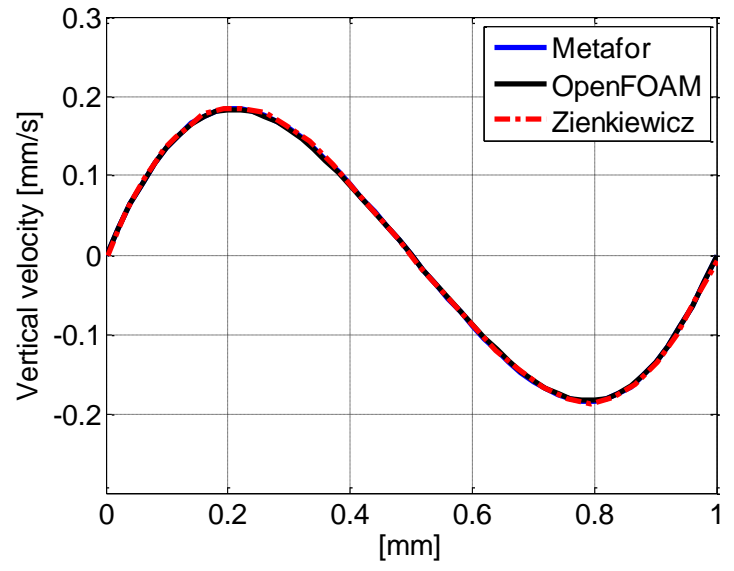

Figure 5. Vertical velocity profile on the horizontal line in the middle of the geometry. 


\subsubsection{Plane slide pad bearing}

The second application is a well-known configuration in tribology: the plane slide pad bearing. It is made of two non-parallel edges: the bottom one has a horizontal motion at a speed $v_{0}(10 \mathrm{~m} / \mathrm{s}$ to the right in the present case) while the top edge is fixed (see Figure 6). Fluid flows in through the left side which has a thickness $h_{1}$ and leaves the domain through the right edge $h_{2}$.
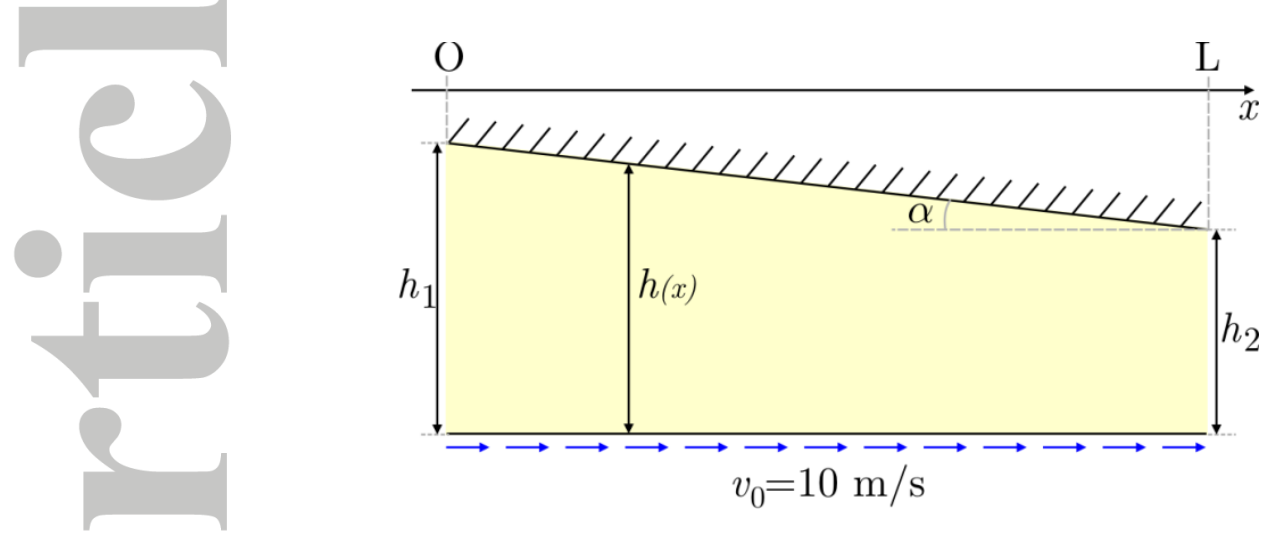

Figure 6. Geometry and boundary conditions of the plane slide pad bearing test: $h_{2}=0.05 \mathrm{~mm}$ and $O B=10 \mathrm{~mm}$.

The motion of the fluid in the converging gap induces an hydrodynamic pressure field. The dimensionless pressure profile along OL obtained by integrating the Reynolds equation is given by eq. 15. $[42,43]$

$$
\bar{p}(x)=\frac{6 h_{2}^{2}}{L \tan (\alpha)}\left[\frac{1}{h(x)}-\frac{1}{h^{2}(x)} \frac{h_{1} h_{2}}{h_{1}+h_{2}}-\frac{1}{h_{1}+h_{2}}\right]
$$

Integrating the pressure profile and assuming an out-of-plane width $t$, gives the dimensionless vertical load $\bar{W}$ generated by the system (see eq. 16).

$$
\bar{W}=\frac{6 L t}{(a-1)^{2}}\left(\ln a-2 \frac{a-1}{a+1}\right)
$$

where $a=h_{1} / h_{2}$

The non-dimensional pressure field computed over $\mathrm{OL}$ is compared to the corresponding analytical solution for three values of $a$ in Figure 7. Even though the shape of the curve is much influenced by $a$, the numerical results match perfectly the theoretical solution.

The non-dimensional load computed for different values of $a$ is compared to the analytical solution in Figure 8. The results obtained with the FE model follow the analytical curve. 


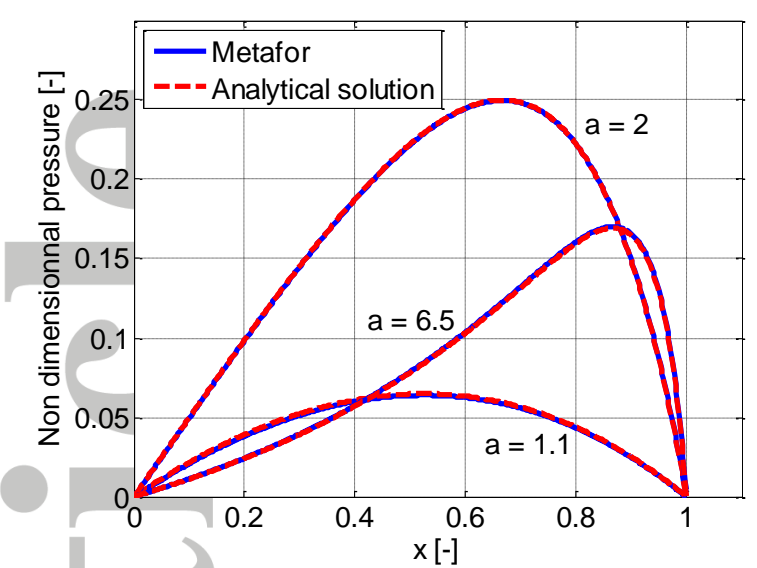

Figure 7. Non-dimensional pressure profile over $\mathrm{OL}$ for three values of $a$ : comparison of the FE results to the analytical solution.

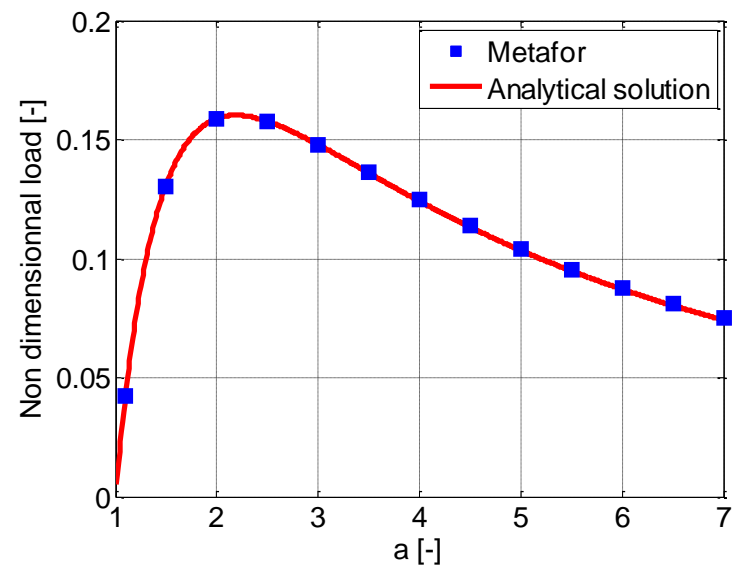

Figure 8. Non-dimensional load as a function of $a$ : comparison of the FE results to the analytical solution.

\subsection{Fluid-structure interaction simulation}

The simulations discussed above show the validity of the present approach when dealing with a Newtonian fluid flow. However, the modelling of MPH lubrication in plane strip drawing requires to model interactions between fluid and solid. Thus, a test case modelling the fluid flow around a flexible beam [31] is conducted to ensure the feasibility of such type of simulation in the FE code Metafor.

\subsubsection{Geometry and boundary conditions}

The problem consists of a fluid flow in a converging channel with a flexible solid beam just before the contraction (see Figure 9). The beam is made of a linear elastic material which has a Young's modulus of $E_{\mathrm{S}}=2.3 \mathrm{MPa}$ and a Poisson's ratio $v_{\mathrm{S}}=0.45$. Large displacement and rotations are taken into account using an hypoelastic formulation involving a Jaumann derivative of the stress tensor. The fluid is a silicon oil. It has a Newtonian behaviour with a constant viscosity $\eta_{\mathrm{F}}=0.145 \mathrm{~Pa}$.s, a density $\rho_{\mathrm{F}}=956 \mathrm{~kg} / \mathrm{m}^{3}$ and a bulk modulus $K_{\mathrm{F}}=1700 \mathrm{MPa}$.

The imposed fluid velocity profile at the entry of the domain has a parabolic shape. The maximal velocity varies according to the following law:

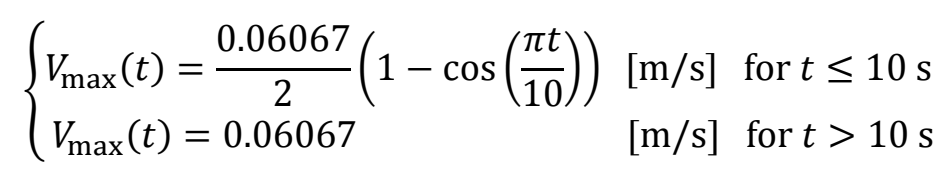

Boundary conditions are depicted in Figure 9. No-slip boundary conditions are considered between the fluid and the beam as well as on the lower edge of the channel while symmetry conditions are applied on the upper edge of the fluid domain.

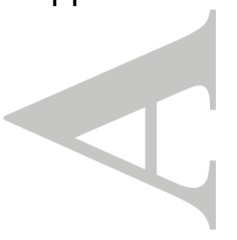




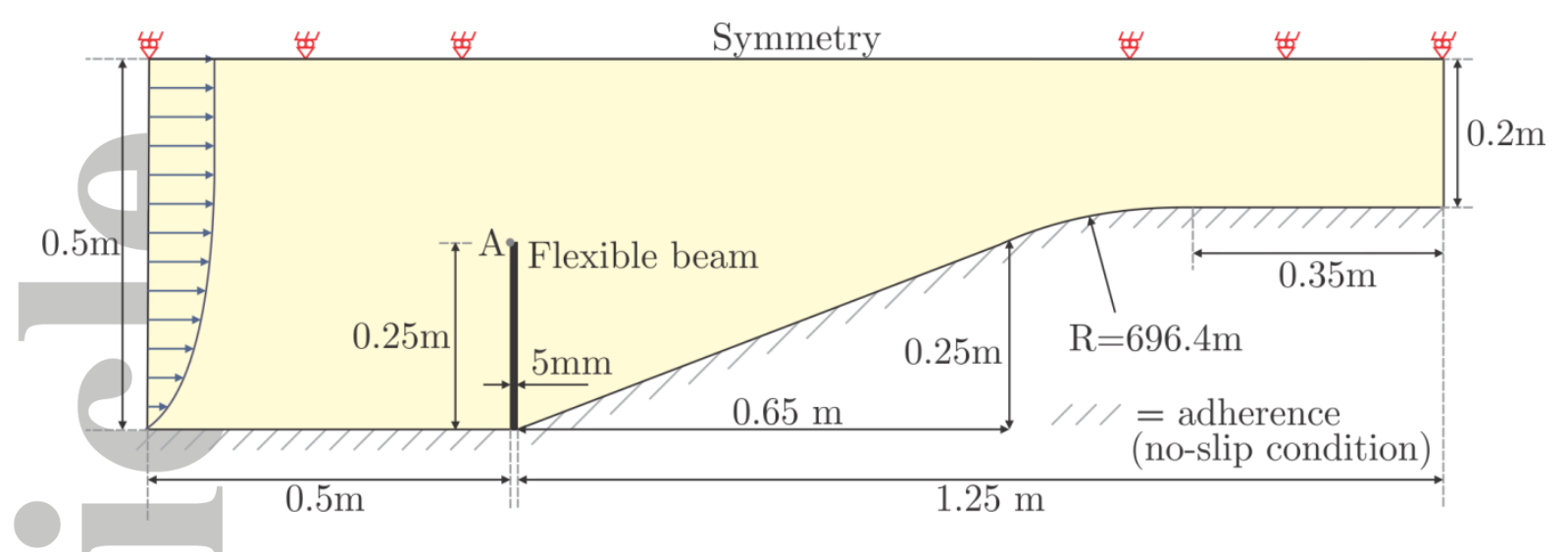

Figure 9. Geometry and boundary conditions used in the FSI test: a flexible beam made of rubber located in a converging channel interacts with a silicon oil flowing from the left to the right.

\subsubsection{Element type and mesh management in ALE}

In this test, the fluid flow induces a bending of the beam which in turns generates a recirculation zone downstream the beam.

A monolithic approach is used in the present study. This means that the fluid flow and the solid deformations are solved simultaneously. Therefore their mutual influence is taken into account at each time step of the simulation.

The elements used for the fluid part are Q1P0 hybrid finite elements while the flexible beam is meshed with $3 \times 30$ EAS elements (Enhanced Assumed Strain - Glaser and Armero [46], Simo et al. [47] - Bui et al. [48] - Adam and Ponthot [49] for the implementation of this element type in Metafor). These elements allow computing precisely the deformation due to bending with a small number of elements through the thickness.

To manage the large deflection of the beam and to speed up the remeshing stage in the ALE procedure, a transfinite mesh is used for the whole domain (see Figure 10). To achieve this, the geometry of the fluid part is divided into 7 sub-domains numbered from I to VII in Figure 11.

The $\mathrm{V}$ and $\mathrm{VI}$ areas are purely Eulerian. This implies that, at the end of each time step, the nodes are moved back to their initial position. On the left vertical edge, node $\mathrm{N}_{\mathrm{A}}$ follows the vertical movement of the upper left corner of the beam while nodes $N_{B}$ and $N_{C}$, located on the symetry axis, follow the horizontal movement of the beam corners. Lines $I_{1}, I_{2}, I_{3}$ and $I_{4}$ are then remeshed by preserving the initial curvilinear abscissa of the nodes. The transfinite mesher is then used to generate the mesh of the subdomains I, II, III, IV and VII. Thanks to this methodology, the elements of the mesh preserve a regular shape throughout the simulation, even when the beam deflection is large. 


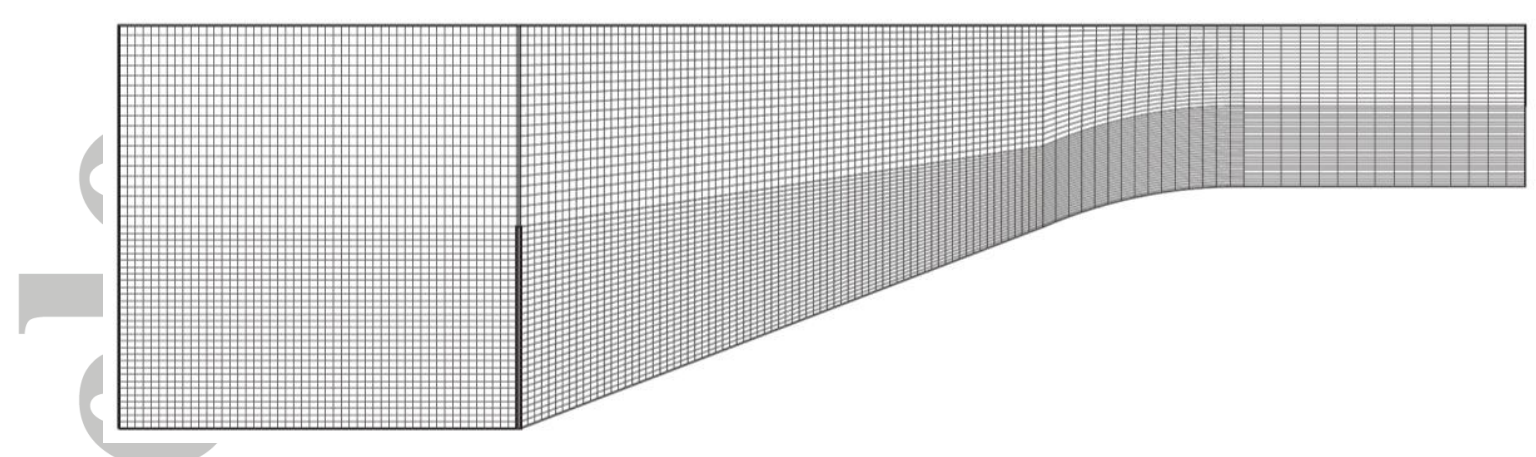

Figure 10. Transfinite mesh made of 7900 quadrangular elements. The flexible beam is discretised with $3 \times 30$ elements.

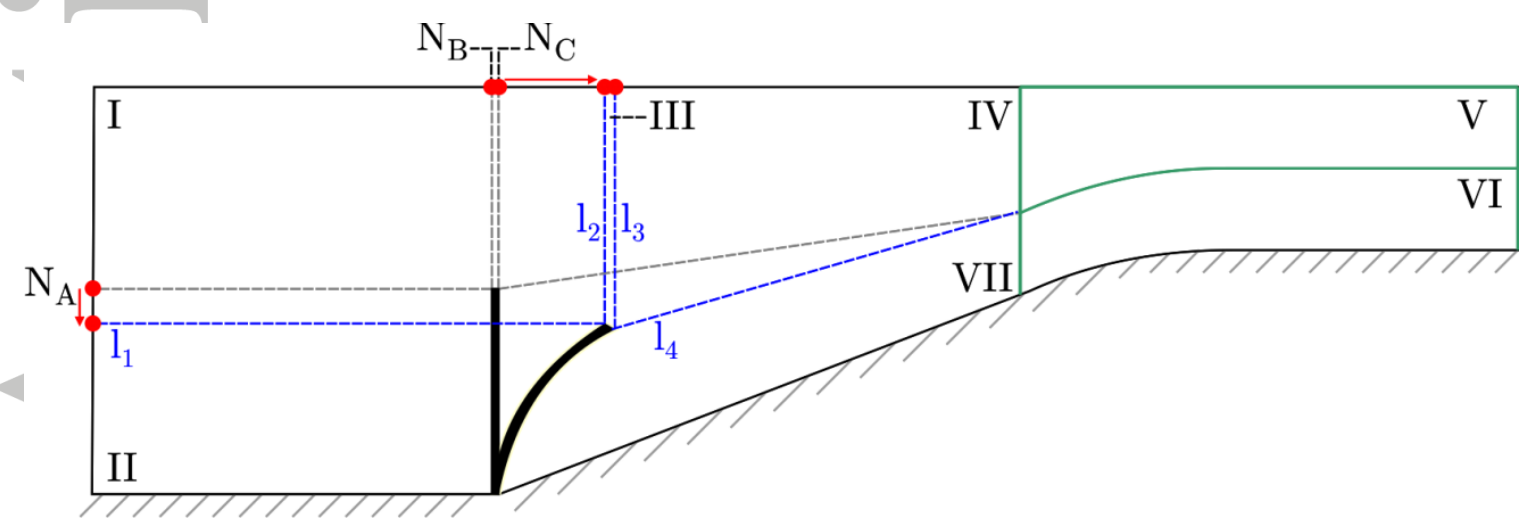

Figure 11. Division of the area covered by the fluid into 7 sub-domains each of them being remeshed with a transfinite mesher in order to preserve regular shape for all the elements throughout the simulation.

\subsubsection{Comparison with a reference solution}

A different methodology from the monolithic approach would be to use a partitioned (staggered) scheme where fluid and solid equations are solved separately in two different pieces of code. This kind of technique includes two groups. First, the fully explicit partitioned coupling schemes where fluid and solid problems are solved successively with simple exchange of boundary conditions. This approach offers a lot of flexibility regarding the choice of solvers but it suffers from poor convergence properties [50]. The second group is made of implicit partitioned schemes which are more robust since they imply several exchanges between fluid and solid codes for each time step of the simulations. That fluid-structure interaction loop is repeated until a convergence criterion, usually defined by a change in displacement, is reached [50].

An implicit partitioned method has been used by Neumann [31] and Degroote [32] to solve the converging channel problem. They recorded the displacement and pressure at point A (see Figure 9) during the simulations as well as the streamlines in the fluid.

The horizontal displacements of point A computed with the present FE approach is identical to the reference solution of Degroote until $t=7.5 \mathrm{sec}$ (see figure Figure 11). Then it underestimates slightly the displacements to match the final displacement predicted by Degroote $(t=25 \mathrm{sec}$ ).

In terms of pressure at point $A$, the 3 curves have the same shape and the present approach gives a result between Neumann and Degroote's solution. In this case, the FE curves is closer to Neumann's solution. Streamlines corresponding to three successive moments of the simulation are represented in Figure 14. The present FE results have the same shape as those from the numerical solution of 
Neumann. For $\mathrm{t}=10 \mathrm{sec}$, a recirculation zone appears after the flexible beam. At $t=25 \mathrm{sec}$, a double recirculation zone exists which is consistent with Neumann's results.

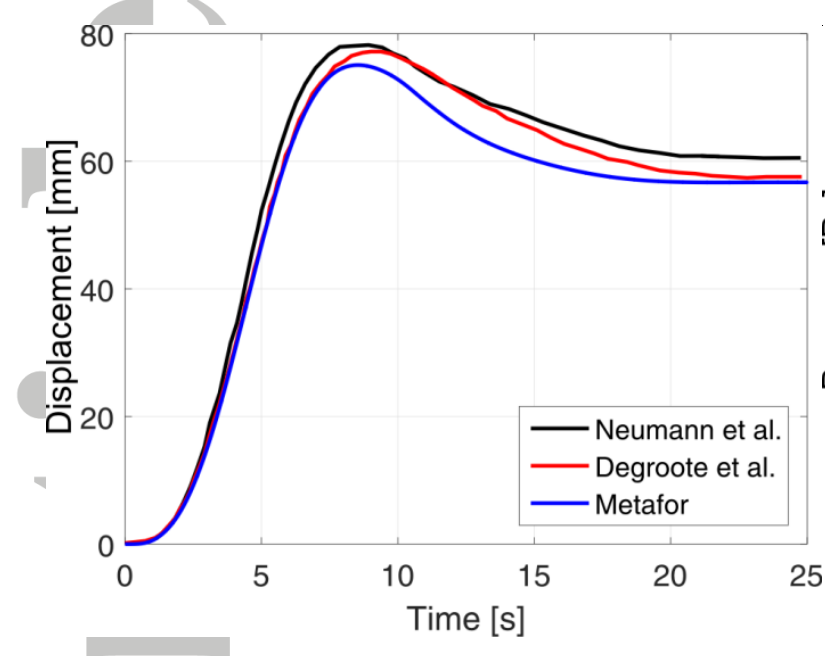

Figure 12. Horizontal displacement of point A (see Figure 9). Metafor results are close to two reference solutions from the literature (Neumann et al. [31] and Degroote et al. [32]).

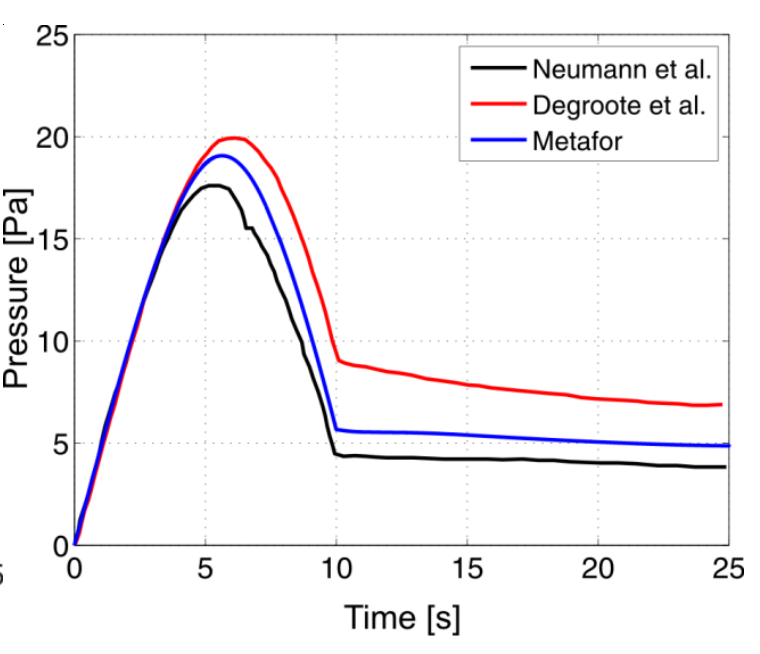

Figure 13. Pressure in the fluid at point A (see Figure 9). Metafor results are close to two reference solutions from the literature (Neumann et al. [31] and Degroote et al. [32]).

\subsection{Partial conclusion}

The goal of this section was to demonstrate the capability of the FE code Metafor to compute fluid flows. A Norton-Hoff material is used to model the behaviour of a Newtonian fluid. The velocity fields and pressure fields have been validated on several configurations by making comparison with reference solutions. For all the purely fluid cases investigated in the present study, when there is no degree of freedom associated to a solid part, a very good agreement has been reached.

The last application discussed in this section deals with a FSI simulation where a flexible beam interacts with the surrounding fluid flow. The results are consistent with reference solutions from the literature in terms of streamlines over the fluid domain.

Yet, there are some differences regarding the pressure and displacement evolutions: the trends and the order of magnitudes are good but the computed values are not identical including among the reference solutions. These differences might be explained by several factors. First of all, the approach used here is monolithic and exact continuity (to the discretization error) between fluid and solid interface is guaranteed since they have both unique nodal positions) whereas Neuman and Degroote employ an iterative staggered scheme. In the latter case, the continuity between fluid and solid domains is approximated by a given tolerance. This might induce some slight errors during the simulation.

Second, the mesh resolution used is also important: the mesh employed with Metafor is much finer than the one employed by Degroote (Neuman does not present his mesh). Also, slight geometry changes might also affect the final solution. Indeed, Degroot does not use a filet between the inclined edge and the horizontal edge close to the channel exit whereas Neumann does not mention the length of the horizontal lower edge close to the exit. 
Regarding these uncertainties, the present validation is only qualitative: the order of magnitude and the observed trends are in agreement with Neumann's and Degroote's solutions. However, this test case is still valuable since it demonstrates the fact that the present approach is capable of handling a FSI problem including large solid displacements in a robust way.

The experience obtained from the setting up of the cases presented in this section has been used to develop the numerical model of Bech's experiments described in the following section. 

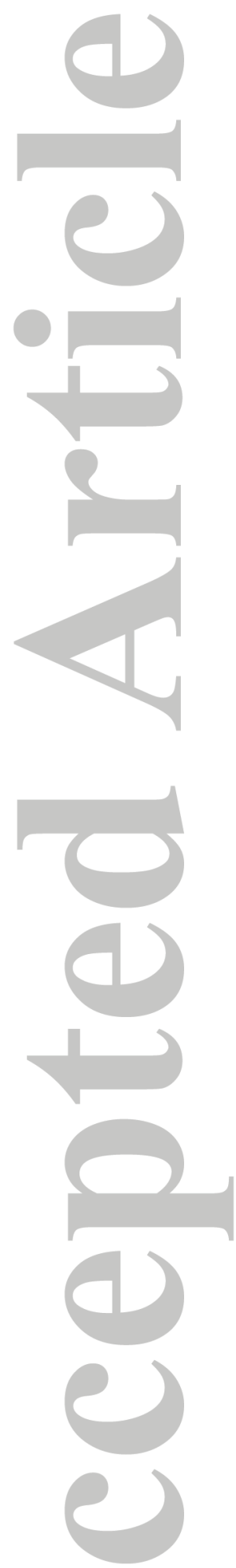
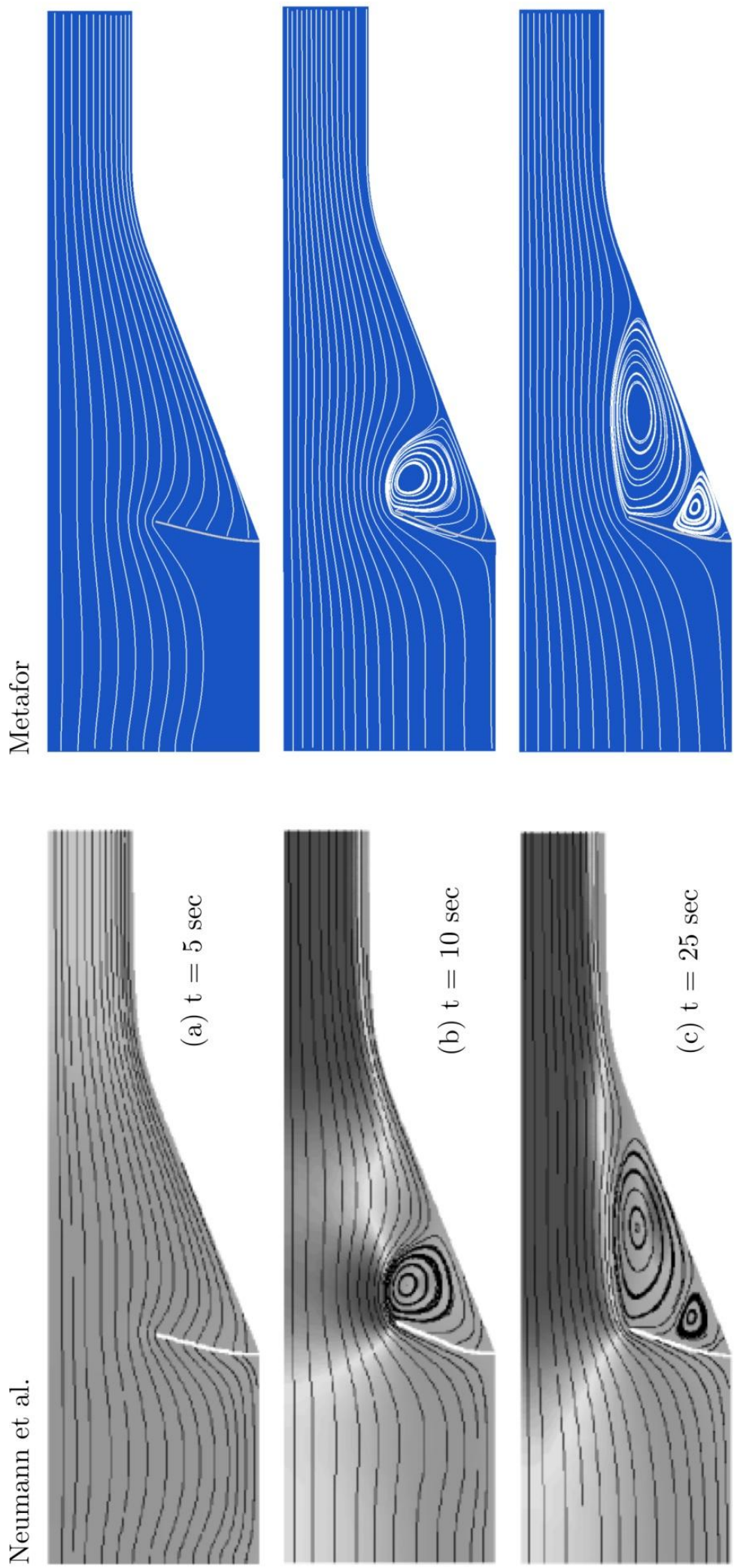

Figure 14. Streamline for different moments of the flow: comparison of Metafor results to the numerical solution from Neumann et al. [31] $]^{1}$. Results computed with the present approach are very similar to the reference solution.

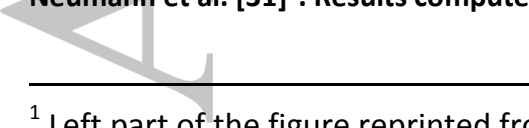

\footnotetext{
${ }^{1}$ Left part of the figure reprinted from Notes on Numerical Fluid Mechanics and Multidisciplinary Design, Vol 91(2), Neumann M., Tiyyagura S.R., Wall W.A., Ramm E., Robustness and efficiency aspects for computational fluid structure interaction, Page 99-114, Copyright (2006), with permission from Springer.
} 


\section{Numerical modelling of micro plastohydrodynamic lubrication in plane strip drawing}

\subsection{Introduction}

This chapter presents a FE model developed to numerically reproduce the experiments conducted by Bech. As mentioned in the introduction, Bech [4] pointed out MPH lubrication flows using plane strip drawing. The experimental setup he used is represented in Figure 15. He performed pyramidal indentations on a $1.95 \mathrm{~mm}$ thick aluminium strip and filled them with lubricant before drawing the strip through the die at a given speed $V_{x}$. Thanks to a tool made of glass Bech observed the behaviour of the lubricant cavities while pulling the strip through the tool.

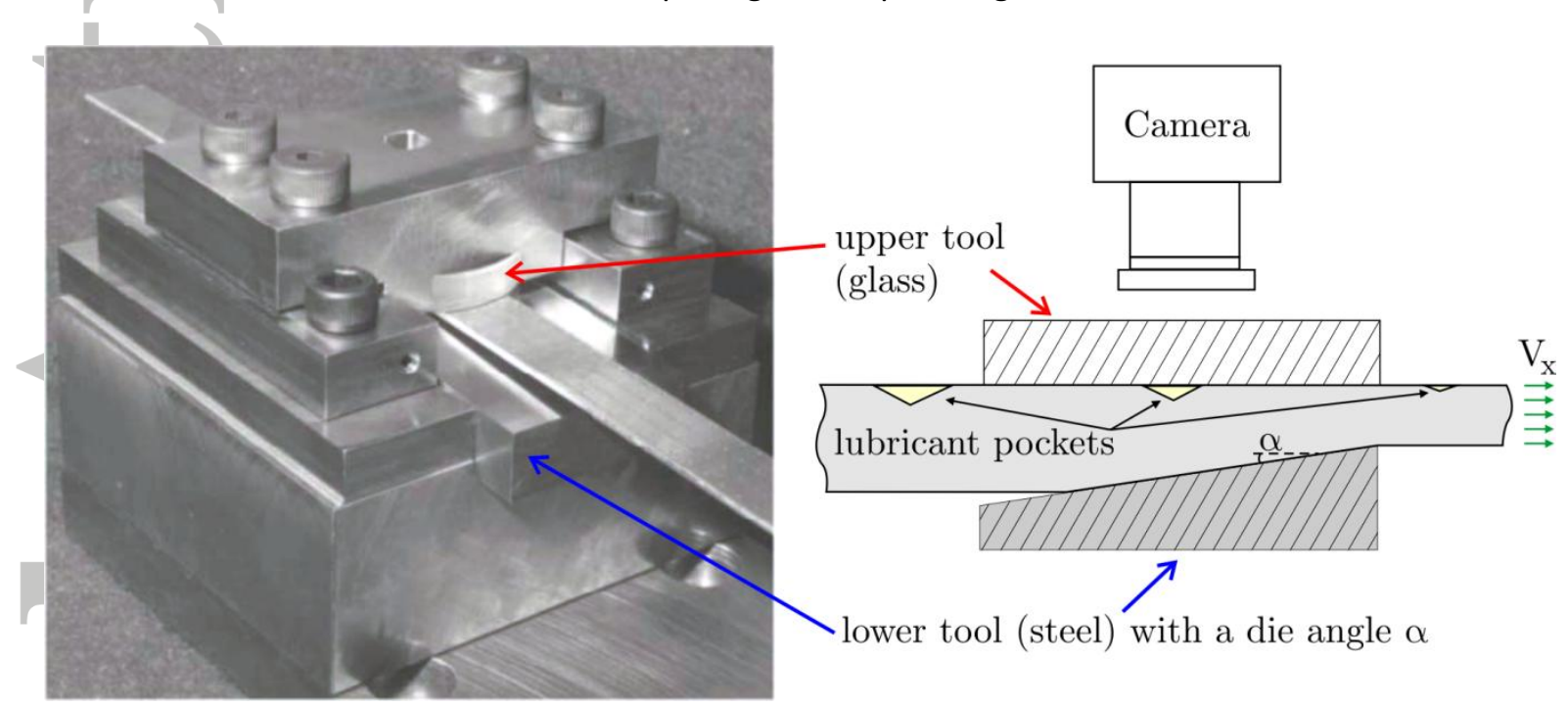

Figure 15. Left: Experimental setup used by Bech to observe MPH lubrication. Right: schematic view of the setup.

Bech investigated the effect of the die angle, the back tension, the strip material behaviour and the friction between the lower surface of the strip and the tool. Figure 16 shows the marks left by the lubricant around the pocket after the drawing process. These marks are essentially oriented in the drawing direction but in some cases transverse flows occur (see for example tests labelled A1 - B5 B7 - C5 - C7 in Figure 16). The phenomenon leading to lubricant escapes at the front is called MicroPlasto-HydroStatic Lubrication (MPHSL) while the term Micro-Plasto-HydroDynamic Lubrication (MPHDL) is used to point out lubricant escapes at the rear of pockets. This terminology will be justified later.

This article is protected by copyright. All rights reserved. 


\begin{tabular}{|c|c|c|c|c|c|c|}
\hline $\begin{array}{l}\text { lubricant } \\
\text { viscosity }\end{array}$ & $\begin{array}{l}\text { drawing } \\
\text { speed }\end{array}$ & reduction & die angle & \begin{tabular}{|l|} 
back \\
tension
\end{tabular} & $\begin{array}{l}\text { strain hard. } \\
\text { exponent }\end{array}$ & \begin{tabular}{|l} 
coefficient \\
of friction
\end{tabular} \\
\hline $\begin{array}{l}\eta=5 \mathrm{cSt} \\
\mathrm{A} 1\end{array}$ & $\begin{array}{l}\text { a } \\
0.2 \mathrm{~mm} / \mathrm{sec} \\
\mathrm{A} 2\end{array}$ & $\begin{array}{l}\text { A } \\
10 \% \\
\text { A3 }\end{array}$ & $\begin{array}{ll} & \\
2^{\circ} \\
\text { A4 }\end{array}$ & $\begin{array}{ll} & \\
0 & \\
\text { A5, REF }\end{array}$ & $\begin{array}{l}n=0.08 \\
\text { A6, REF }\end{array}$ & $\begin{array}{l}\mu \\
\mu=0.025 \\
\text { A7, REF }\end{array}$ \\
\hline $\begin{array}{l}\eta=126 \mathrm{cSt} \\
\mathrm{B} 1, \mathbf{R E F}\end{array}$ & $\begin{array}{l}\text { D mm/sec } \\
\text { B2, REF }\end{array}$ & $\begin{array}{l}\text { B } \\
15 \% \\
\end{array}$ & $\begin{array}{l}3^{\circ} \\
\text { B4, REF }\end{array}$ & $\begin{array}{l}62 \mathrm{~N} / \mathrm{mm}^{2} \\
\mathrm{~B} 5\end{array}$ & $\begin{array}{l}\mathrm{n}=0.16 \\
\mathrm{~B} 6\end{array}$ & $\begin{array}{l}\mu=0.05 \\
\text { B7 }\end{array}$ \\
\hline $\begin{array}{l}\eta=700 \mathrm{cSt} \\
\mathrm{C} 1\end{array}$ & $\begin{array}{l}5 \mathrm{~mm} / \mathrm{sec} \\
\mathrm{C} 2\end{array}$ & \begin{tabular}{|l}
$\mid$ \\
$20 \%$ \\
C3, REF \\
\end{tabular} & $\begin{array}{l}5^{\circ} \\
\mathrm{C} 4 \\
\end{array}$ & $\begin{array}{l}105 \mathrm{~N} / \mathrm{mm}^{2} \\
\text { C5 }\end{array}$ & $\begin{array}{l}\mathrm{n}=0.21 \\
\mathrm{C} 6\end{array}$ & $\begin{array}{l}\mu=0.28 \\
\mathrm{C} 7\end{array}$ \\
\hline
\end{tabular}

Figure 16. Imprints left by the lubricant on the strip after it escapes from the pyramidal cavities during plane strip drawing in Bech's experiments [4] (drawing direction: from left to right). The effect of each parameter is studied one by one and the results are compared to a unique reference test denoted REF $^{2}$.

Bech also measured roughness increase where MPH lubrication occurred. The order of magnitude of the peak-to-valley distance in these measurements is 2-5 $\mu \mathrm{m}$. According to Bech, this gives an estimate of the film thickness on the plateaus when MPH lubrication takes place.

Such a large difference between the inlet strip thickness $(1.95 \mathrm{~mm})$ and the lubricant film thickness on the plateaus is one of the biggest challenges to tackle. To do so, a multiscale approach is developed. It consists in running successively two numerical models.

The first one, named "full model" deals with a whole strip pulled through rigid-tools. A lubricant cavity, present at the strip surface, interacts with the elastoplastic deformation of the strip. During this simulation, the displacements of a rectangular area around the cavity are recorded. They are then used as boundary conditions in a second model named "reduced model". This model focuses on a smaller region around the cavity and is capable of predicting lubricant escapes with a film thickness lower than a micrometre.

These two models are developed with Metafor, an in-house nonlinear FE code [30], under 2D plane strain hypothesis. The models are discussed in the two sections below: the geometry, boundary conditions and the mesh are presented as well as the numerical results.

\subsection{Full model - macroscopic simulation}

\subsubsection{Geometry}

The geometry of the full model is represented in Figure 17. The aluminium strip is pulled between rigid tools at a constant speed $V_{x}$. The upper tool is horizontal while the lower one has a slope $\alpha$. A single lubricant pocket is located at the strip surface ahead of the reduction zone. In this way, the

\footnotetext{
${ }^{2}$ Figure reprinted from Wear, Vol 232(2), Bech J, Bay N, Eriksen M., Entrapment and escape of liquid lubricant in metal forming, Page 134-139, Copyright (1999), with permission from Elsevier.
}

This article is protected by copyright. All rights reserved. 
cavity reaches the reduction zone once a steady state solid-to-solid contact pressure profile is achieved.

The pyramidal cavity of Bech's experiments is modelled as a triangle surmounted by a thin rectangle. The rectangle height is chosen as 1 percent of the triangle depth to minimise the impact of this geometry modification. The reason for this is to achieve a regular mesh - made of quadrangular elements - with a simple transfinite mesher all along the simulation. Otherwise, the mesh would contain at least two triangular or degenerated quadrangular elements at both ends of the cavity which would cause numerical problems in the simulation.

As in Bech's experiments, the slope of the cavity is $10^{\circ}$ and its width is $1 \mathrm{~mm}$ (respectively Өand $I_{\mathrm{c}}$ in Figure $17-b$ ). The geometrical dimensions used when modelling the $\mathrm{C} 7$ test conditions are listed in Tableau 1.

a) Overview of the geometry

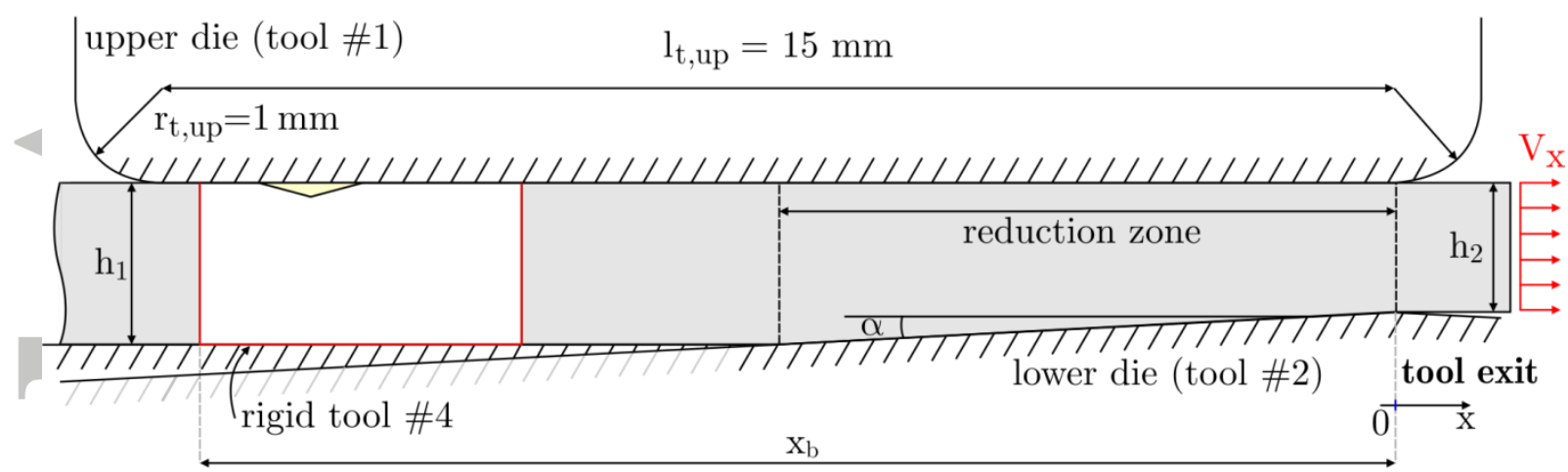

b) Zoom on the rectangular area surrounding the lubricant cavity

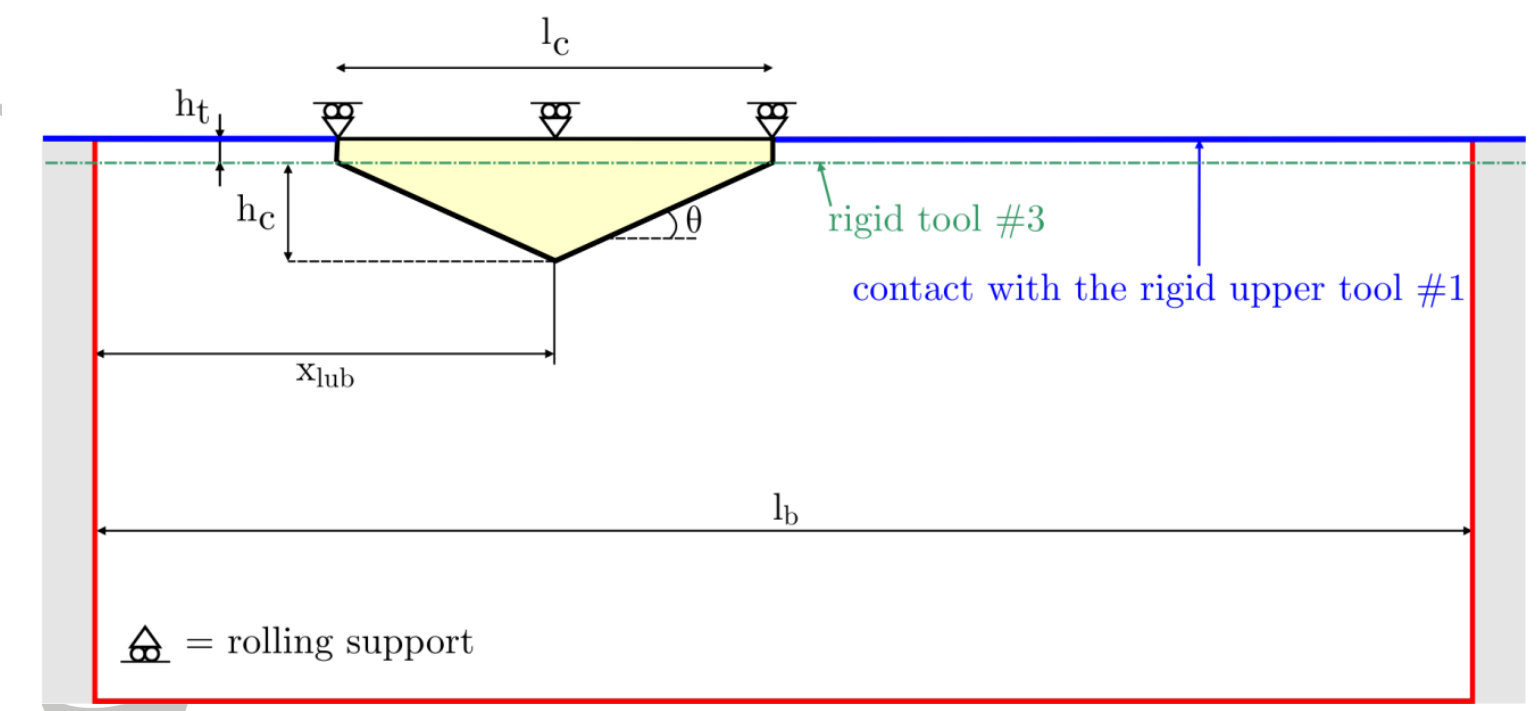

Figure 17. Geometry of the plane strip drawing model (full model). Rigid tools \#3 and \#4 are numerical artefacts which prevents respectively fluid element distortions and the strip bending due to the tool asymmetry. These artefacts help to manage the complex interactions between the fluid cavity and the elastoplastic deformation of the strip as well as the conditions applied on the fluid upper edge. (nb: a fillet having a curvature radius of $1 \mathrm{~mm}$ is used to ensure the normal continuity on the lower tool at the apex of the tool exit.) 


\begin{tabular}{|c|c|c|c|c|c|c|c|c|c|c|}
\hline \multirow{2}{*}{$Z$} & $\begin{array}{c}\mathrm{h}_{1} \\
{[\mathrm{~mm}]}\end{array}$ & $\begin{array}{c}\text { Red } \\
{[\%]}\end{array}$ & $\begin{array}{c}\alpha \\
{\left[{ }^{\circ}\right]}\end{array}$ & $\begin{array}{c}\mathrm{V}_{\mathrm{x}} \\
{[\mathrm{mm} / \mathrm{s}]}\end{array}$ & $\begin{array}{c}\mathrm{I}_{\mathrm{b}} \\
{[\mathrm{mm}]}\end{array}$ & $\begin{array}{c}\mathrm{x}_{\mathrm{lub}} \\
{[\mathrm{mm}]}\end{array}$ & $\begin{array}{c}\mathrm{h}_{\mathrm{c}} \\
{[\mathrm{mm}]}\end{array}$ & $\begin{array}{c}\mathrm{h}_{\mathrm{t}} \\
{[\mathrm{mm}]}\end{array}$ & $\begin{array}{c}\mathrm{I}_{\mathrm{c}} \\
{[\mathrm{mm}]}\end{array}$ & $\begin{array}{c}\Theta \\
{\left[{ }^{\circ}\right]}\end{array}$ \\
\cline { 2 - 9 } & 1.95 & 20 & 3 & 0.5 & 5 & 1.5 & 0.088 & $0.01 \times \mathrm{h}_{\mathrm{c}}$ & 1 & 10 \\
\hline
\end{tabular}

Tableau 1. Parameters corresponding to the $\mathrm{C7}$ test condition investigated by Bech [4] (see also Figure 16). In this case, an escape of lubricant at the front of the pocket was experimentally observed (MPHSL) and is thus expected in the numerical model

\subsubsection{Material behaviour}

The aluminium strip elastoplastic behaviour is modelled with the following hardening law:

$$
\sigma_{Y}=\left(1875.8+21.98 \bar{\varepsilon}^{p l}\right)\left(1-0.042 \exp \left(-13.28 \bar{\varepsilon}^{p l}\right)\right)-1584.2 \mathrm{MPa}
$$

Numerical parameters are identified on experimental data obtained by Bech with plane strain compression tests.

The fluid is assumed to be Newtonian with a constant viscosity $\eta=35.06$ Pa.s which is based on Bech's estimation [4]. A constant bulk modulus $\mathrm{K}=1700 \mathrm{MPa}$ is used to compute the pressure increase in the lubricant due to the thickness reduction of the strip.

Accounting for such different materials behaviour (aluminium and oil) in the same model is another challenge. Indeed, if we compare an estimation of the shear stress in the cavity $\left(\tau_{F}=\eta V_{x} /\right.$ $h_{c} \sim 200 \mathrm{~Pa}$ ) to the shear bulk modulus of aluminum ( $\mathrm{G}=26 \mathrm{GPa}$ ) : there is a difference of 8 orders of magnitude between these two values. Extra care must be taken when defining the boundary conditions applied to the model. These are discussed in the next paragraphs.

\subsubsection{Boundary conditions}

The strip motion is induced by prescribed horizontal displacements at a constant speed $V_{x}$ applied on the right edge of the strip (see Figure 17).

Four rigid contact tools are used in the simulations. The upper one (tool \#1 in Figure 17) has a horizontal length of $15 \mathrm{~mm}$ and two $1 \mathrm{~mm}$-radius fillets located at both ends.

The lower tool (\#2) is made of two straight lines having an angle $\alpha$ compared to the horizontal. These lines are linked by a $1 \mathrm{~mm}$-radius fillet located at the tool exit. These two contact tools are representative of the experimental device.

For the purpose of the simulations, two contacts tools were added: one at the bottom of the strip (tool \#4) and another one close to the top of the strip (tool \#3).

Experimental observations show that the strip thickness reduction induces a shrinkage of the cavity width. This means that the bottom edges of the cavity are moving up and eventually could come into contact with the upper tool. If this happens in the numerical model, this means that, due to the penalty algorithm used to enforce contact conditions that allows - small - interpenetrations, the thickness of the fluid film will become negative! In order to avoid such a physical nonsense, a rigid tool (tool \#3) has been added at a distance $h_{t}$ from the upper tool (tool \#1) ) which enforces a minimal fluid thickness of $h_{t}$. This is of course a numerical artefact, but $h_{t}$ is always kept very small with respect to the cavity depth and, as will be shown later, the practical choice of $h_{t}$ does not influence much the obtained results. 
The horizontal tool at the bottom (\#4) is also an artefact which, this time, prevents the bending of the strip at the entry due to the asymmetry of the tool. By doing that, the complex management of the fluid free surface and its contact with the upper tool is avoided.

The contact algorithm is the penalty method. The normal contact force $\left(F_{\mathrm{N}}\right)$ computed with this method, on each slave node, is proportional to the normal penetration (referred to as the normal gap $G_{N}$ in the following paragraphs) between the roll and strip surfaces, the normal penalty $\left(C_{N}\right)$ and the area associated with each slave node $A_{\text {slave }}[51,52]$.

$$
\frac{F_{\mathrm{N}}}{A_{\text {slave }}}=C_{\mathrm{N}} G_{\mathrm{N}}
$$

The normal penalty values used (see Tableau 2) are a compromise between the computational time (the larger the penalty, the larger the computational time) and the normal gap values (the larger the penalty, the smaller the gap).

\begin{tabular}{|c|l|r|c|}
\hline Contact tool & Entity in contact with the tool & $\begin{array}{c}\mathrm{C}_{\mathrm{N}} \\
{[\mathrm{MPa} / \mathrm{mm}]}\end{array}$ & $\begin{array}{c}\text { Coulomb's friction coefficient } \\
\mu_{\mathrm{C}}\end{array}$ \\
\hline$\# 1$ & top of the strip & $510^{7}$ & 0.05 \\
\hline$\# 2$ & bottom of the strip & $10^{6}$ & 0.25 \\
\hline$\# 3$ & lower part of the cavity & $10^{5}$ & 0.05 \\
\hline$\# 4$ & bottom of the strip & $10^{6}$ & 0 \\
\hline
\end{tabular}

Tableau 2. Normal penalty and friction coefficient values used for the different contact interaction.

Friction conditions are modelled with Coulomb's friction law. During his experiments, Bech used different types of lubricant on the bottom and the top of the strip in order to change the shape of the pressure profile and therefore favouring either lubricant escape at the front or at the rear of the lubricant cavity.

In the simulations, friction coefficient values identified by Bech during his test are used. For the C7 test condition studied in this section, $\mu_{1}=0.05$ and $\mu_{2}=0.25$ are employed for the upper surface and the lower surface of the strip respectively.

It is interesting to notice that, when the cavity shrinks, the lower part of the cavity comes into contact with the horizontal tool \#3 and contact is managed as if there was solid-to-solid contact. Therefore, the friction coefficient used there is the same as the one between the upper surface of the strip and the upper tool.

The only part where frictionless contact conditions are applied is the lower horizontal tool \#4: it reduces its influence on the process. Indeed, the tool \#4 has an effect on the contact pressure profile between the upper tool and the strip: since the contact length increases. However, these perturbations are confined to the contact entry region and do not affect the area where MPH lubrication occurs.

The interaction of the upper edge of the fluid and the upper tool are not managed by a contact method but rolling support conditions are applied on this edge. Indeed, due to the low viscosity and low compressibility of the fluid material, small gap variations induce instabilities during Newton Raphson iterations at each time step of the simulations. These perturbations are avoided when the vertical displacement is prescribed. There is no adherence conditions applied to the fluid since the 
flow within the cavity is not our main concern at this stage. The flow in the cavity will be dealt with more precision in the second stage of the 2-scale approach by the "reduced model" described in the next section.

\subsubsection{Mesh}

The mesh is represented in Figure 18. Most of the strip mesh is made of quadrilateral elements having a typical size of $0.05 \mathrm{~mm}$. The goal of such a small size is to reduce the variation in the pulling load, contact pressure profile, etc. due to the nodes entering and leaving the contact zones and to achieve a good discretisation of the contact pressure profile all along the simulation.

The mesh of the fluid domain is generated with a transfinite mesher. The horizontal mesh size follows a geometrical progression in order to preserve a good aspect ratio in the whole fluid domain (see Figure 18-c and d).

The use of a transfinite mesher makes things easier for the remeshing part of the ALE stage. Indeed, as soon as the nodes on the upper edge of the fluid domain are repositioned by a procedure which preserves the initial curvilinear abscissa, the inner nodes position is obtained by activating the mesher again.

From our numerical tests it results that only two elements are needed through the fluid depth. Using more elements leads to a larger computational time and does not provide more accurate results in terms of hydrostatic fluid pressure and strip deformation. Indeed, the goal of this model is not to represent precisely the lubricant flow within the cavity but to compute displacements to be used as boundary conditions in another model.

The transition zone between the regular mesh of the strip ( $80 \%$ of the strip height) and the cavity is obtained with the implementation [30] of the unstructured quadrilateral mesh generation technique presented in [53].

The total number of elements in the mesh represented in Figure 18 is 20085,320 are used for the cavity.

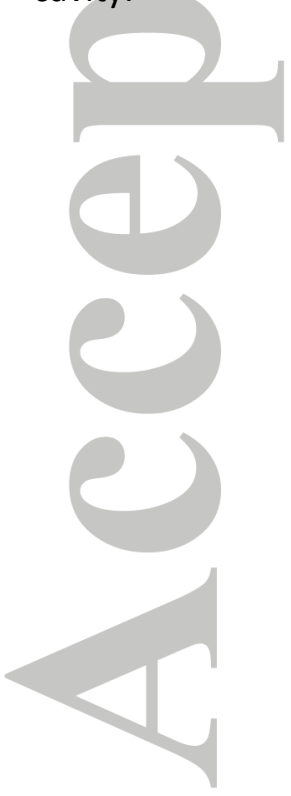

This article is protected by copyright. All rights reserved. 
a) Undeformed mesh : position of the rectangular zone surrounding the lubricant cavity (in red) ahead of the contact zone

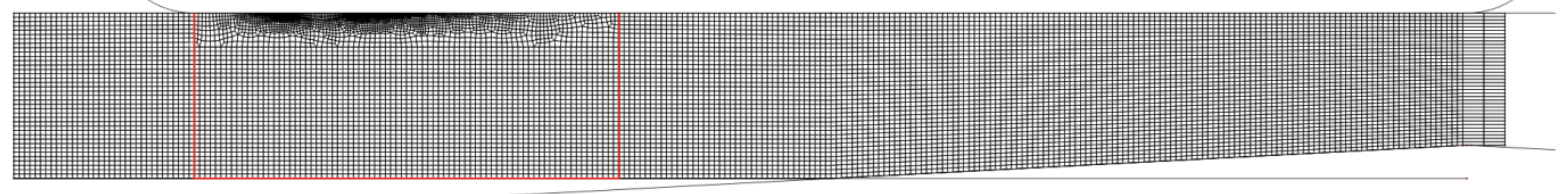

b) Zoom on the rectangular zone surrounding the lubricant cavity

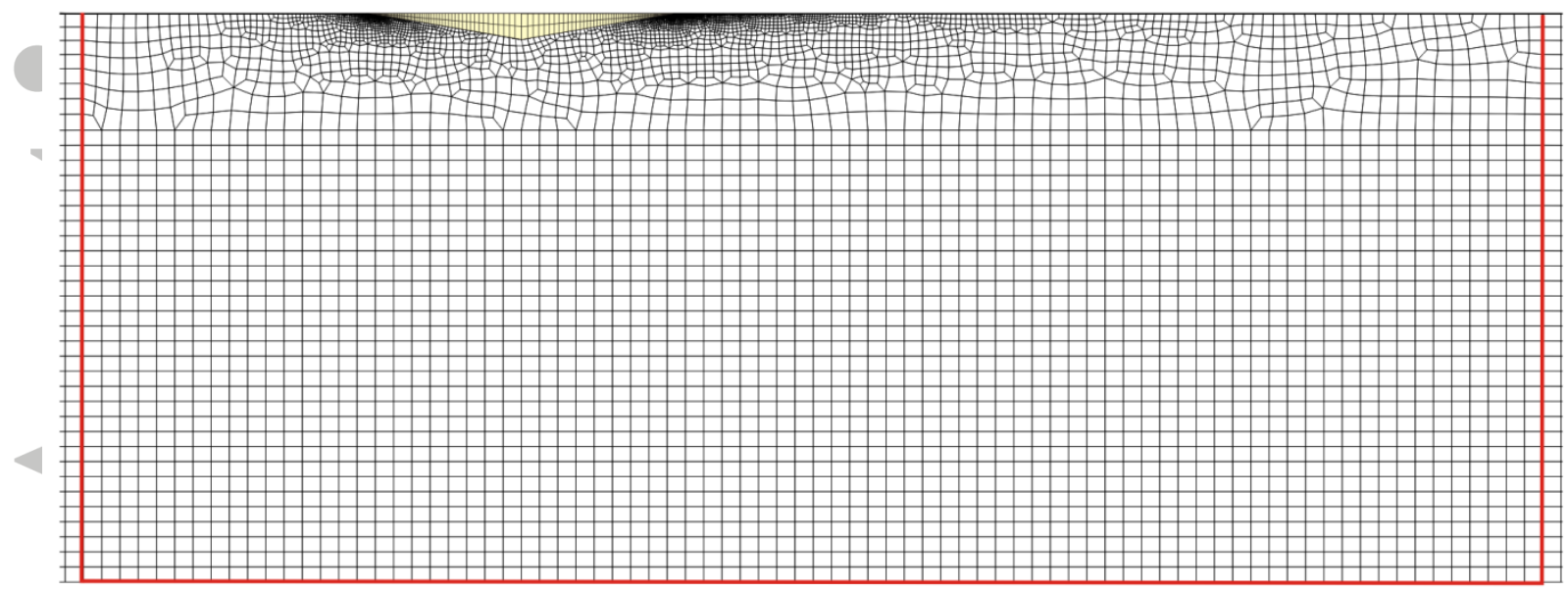

c) Zoom on the lubricant cavity

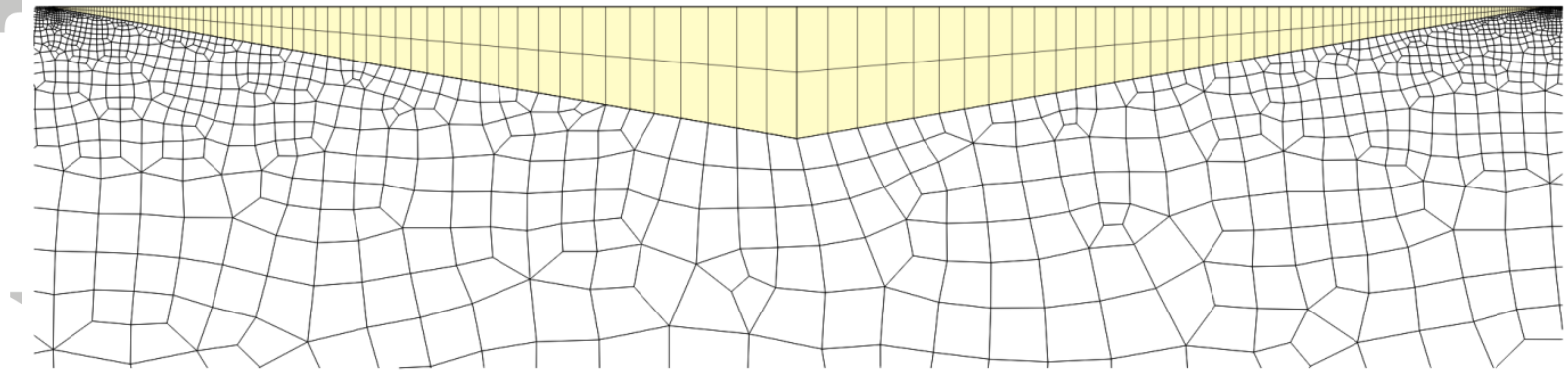

d) Zoom on the right part of the lubricant cavity

rigid tool \#3

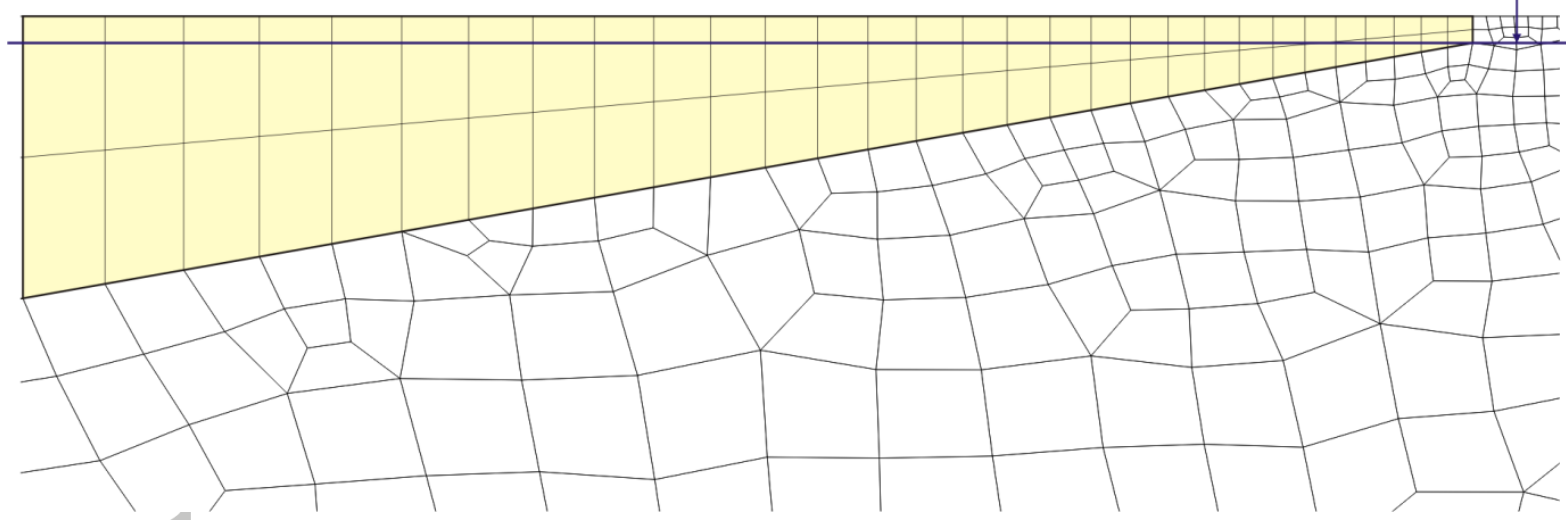

Figure 18. Mesh used when modelling $\mathrm{C7}$ test condition from Bech's experiments with the full model (the lubricant is represented in yellow). 


\subsubsection{Spatial and temporal integration}

The time integration is achieved using a Chung-Hulbert implicit dynamic scheme [36] where the parameters $\alpha_{M}, \alpha_{F}, \beta_{0}, \gamma_{0}$ are respectively set to $-0.97,0.01,0.25$ and 0.5 . For the full model a typical time step is 0.005 and the computational interval is $22.5 \mathrm{sec}$. On the other hand for the reduced model discussed in the next section, a typical time step is $5 \mathrm{E}-5 \mathrm{sec}$. That difference is due to the smaller size of the fluid elements used in the reduced model.

In these simulations, four-node elements are used. A selective reduced integration (SRI) method is performed on these elements. The deviatoric part of the stress increment is computed at four Gauss points. The pressure is assumed constant all over the element: it is computed and stored at a single Gauss point at the element centre. This allows us to avoid both volumetric locking as well as hourglass modes [54].

During the rezoning part of the ALE procedure, the hydrostatic pressure field is transferred from the old mesh to the new mesh using a method called Godunov-type update technique based on a finite volume method [13].

The choices listed above also prevail for the reduced model discussed in the next section.

\subsection{6. $\mathrm{C} 7$ test condition: results analysis}

This model allows us to compare the hydrostatic pressure in the lubricant to the contact pressure profile between the upper die and the solid strip material. During the first part of the simulation, the hydrostatic pressure is lower than the solid-to-solid contact at both ends of the cavity pressure explaining that the cavity remains sealed (see Figure 19-a) and thus there is no fluid flow out of the cavity. Once the front of the pocket is located at $5.2 \mathrm{~mm}$ from the tool exit, the lubricant pressure exceeds the solid-to-solid pressure (see Figure 19-b). The condition allowing a lubricant flow on the plateau at the front of the pocket is thus fulfilled.

This article is protected by copyright. All rights reserved. 
a) Position \#1: the lubricant is trapped in the cavity

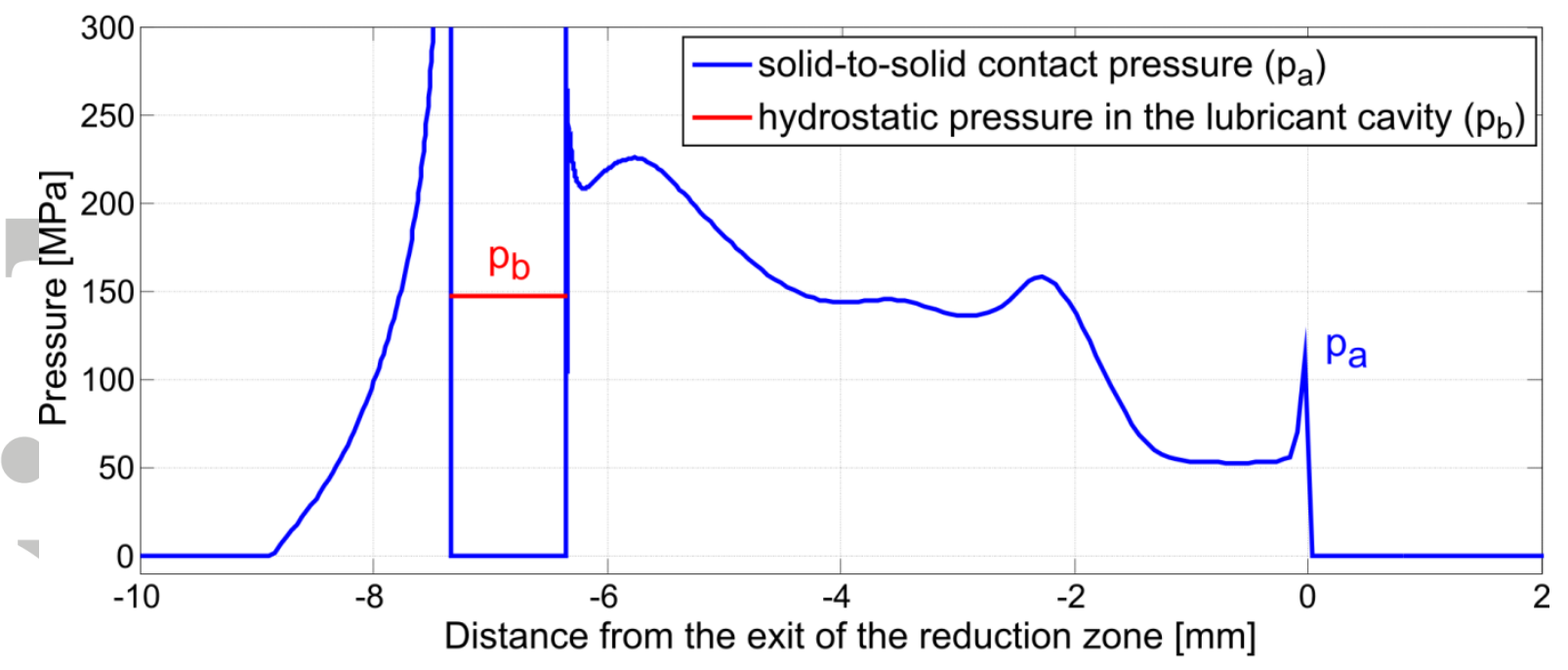

b) Position \#2: the condition allowing lubricant escape at the front of the cavity is met

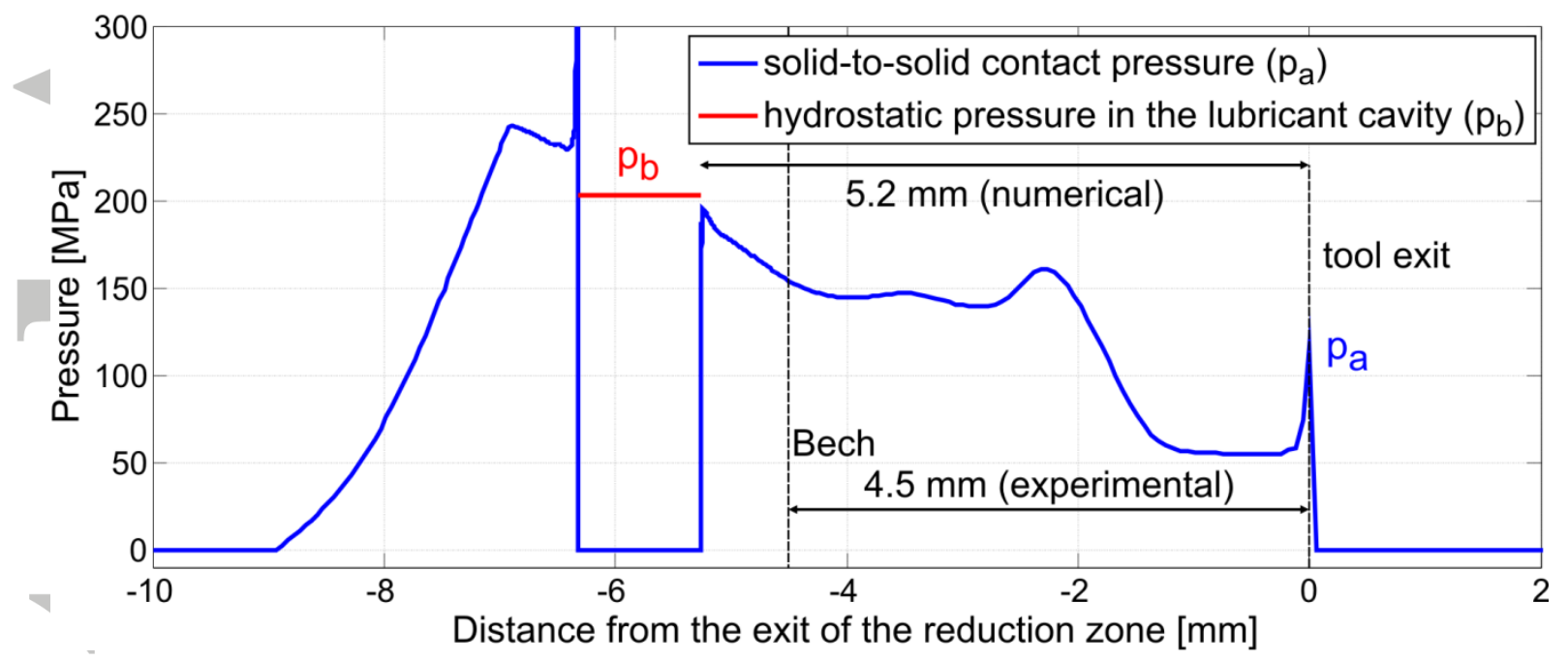

Figure 19. Comparison of the lubricant hydrostatic pressure $p_{\mathrm{b}}$ to the solid-to-solid contact pressure $p_{\mathrm{a}}$ computed for $\mathrm{C7}$ test condition. A) Position 1: the lubricant is trapped in the cavity $\left(p_{b}<p_{a}\right)$. B) Position 2: the condition allowing lubricant escapes $\left(p_{b}>p_{a}\right)$ is achieved at the front of the cavity $5.2 \mathrm{~mm}$ away from the tool exit. This is close to the measurement of Bech [4] $4.5 \mathrm{~mm}$.

In Figure 20, a zoom on the mesh ahead of the cavity when this condition is met exhibits a gap between the mesh of the strip and the tool \#1. This gap is generated by the lubricant pressure and the strip deformation around the lubricant pocket which widens the right edges of the cavity. The strip deformations are themselves affected by the contact conditions, the strip thickness reduction, the pulling speed, etc.

It is clear that there is no pressure transmitted to the upper tool on the edge of the cavity in Figure 20 but the length of this zone is so small that it is negligible with respect to the scale of Figure 19-b, which explains why it is not perceptible in this Figure.

Also, in the configuration represented in Figure 20, tool \#3 has no effect whereas it is required for the configuration presented in Figure 19-a when the solid-to-solid contact pressure is larger than the fluid pressure. 
External load (rolling support)

External load

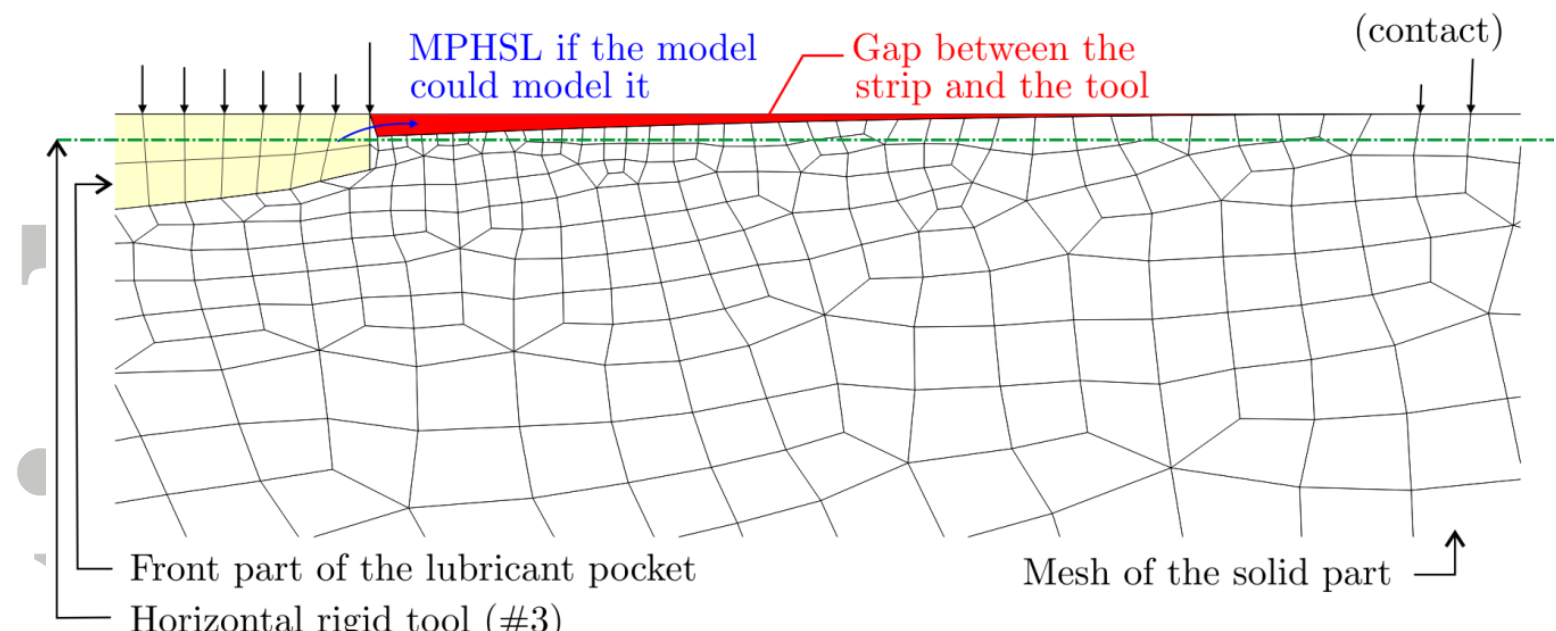

Horizontal rigid tool $(\# 3)$

Figure 20. Zoom on the mesh located on the front part of the lubricant pocket when the condition $p_{\mathrm{b}}>p_{\mathrm{a}}$ is fulfilled. A gap between the strip and the upper tool can be observed (area filled in red). This gap is generated by the lubricant pressure and the elastoplastic deformation of the strip around the cavity which widen the front of the lubricant pocket.

To illustrate that behaviour, Figure 21 compares the initial configuration and final shape of the cavity after the cavity has reached the reduction zone. We can clearly see in that figure that, after deformation, the rear part is closed while the left and right edges are pushed to the right therefore inducing a lubricant cavity escape at the front.

This is due to the large difference in the frictional behaviour at the top and bottom of the strip. In the present case, friction is larger at the bottom. Sliding is thus more constrained, generating a strain gradient through the thickness which induces the pocket shape presented in the Figure 21 favouring lubricant escape at the front.

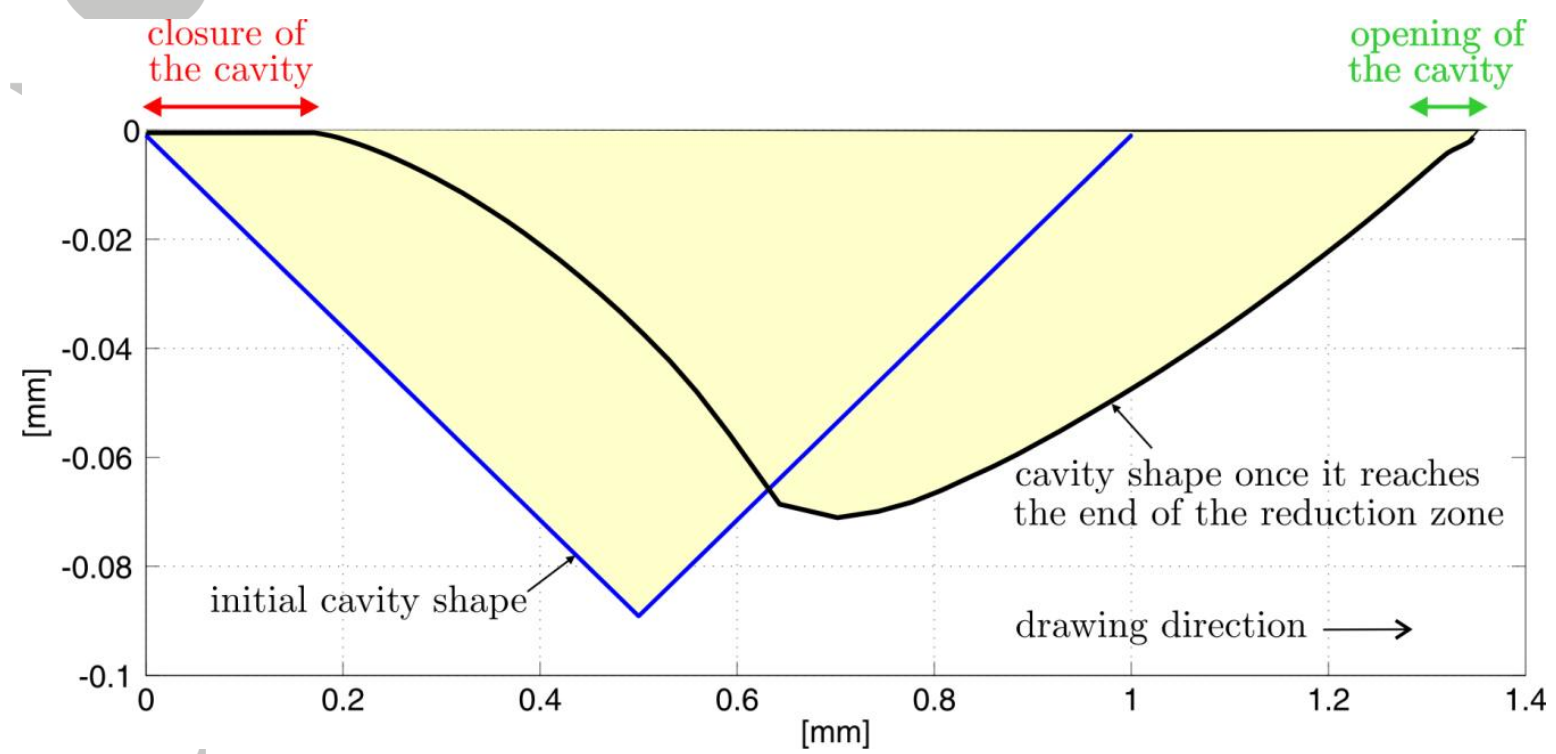

Figure 21. Comparison of the final shape of the cavity once it is drawn through the reduction zone and the initial triangular shape for $\mathrm{C7}$ tests condition. The final cavity shape is such that the lubricant flow occurs at the front while the rear of the cavity is closed. 
The model described above is not realistic beyond the moment when the condition $p_{\mathrm{b}}>p_{\mathrm{a}}$ is met because it does not allow the fluid to escape from the pocket and fill the gap. To take this phenomenon into account a finer model must be used. It is described in the following section.

\subsection{Reduced model}

The aim of the microscopic model is to account for lubricant escapes from the main cavity. To do so, two thin meshed pipes with an initial thickness close to zero are added at both ends of the cavity (as illustrated in Figure 22). Thanks to the use of the Arbitrary Lagrangian Eulerian (ALE) formalism, the lubricant may escape from the triangular cavity to fill the pipes and to lubricate the plateaus.

The model is used to reproduce REF test condition from Bech's experiments (see Figure 16). The test parameters are the same as in the $\mathrm{C} 7$ test condition studied above. The only differences concern the strip thickness reduction which is $21.5 \%$ in the present case and the friction level on top and bottom of the strip which are respectively $\mu_{1}=0.05$ and $\mu_{2}=0.025$. The REF test condition is chosen here since it experimentally highlights both MPHDL and MPHSL lubrication flows.

Thus, another simulation has been conducted with the full model discussed in previous section. Then, these results have been applied as boundary conditions in the reduced model as explained in the following paragraphs.

\subsubsection{Geometry}

The geometry is represented in Figure 22. It consists of a rectangular strip at the top of which is located the fluid domain. This domain is made of a rectangular cavity linked to two very thin rectangular areas (called pipes). The transition between these pipes and the cavity is achieved by two fillets having a radius $r$.

\begin{tabular}{|c|c|c|c|c|c|c|c|c|c|c|}
\hline \multirow{2}{*}{2} & $\begin{array}{c}\mathrm{h}_{\mathrm{b}} \\
{[\mathrm{mm}]}\end{array}$ & $\begin{array}{c}\text { Red. } \\
{[\%]}\end{array}$ & $\begin{array}{c}\alpha \\
{\left[{ }^{\circ}\right]}\end{array}$ & $\begin{array}{c}\mathrm{V}_{\mathrm{x}} \\
{[\mathrm{mm} / \mathrm{s}]}\end{array}$ & $\begin{array}{c}\Theta \\
{\left[{ }^{\circ}\right]}\end{array}$ & $\begin{array}{c}\mathrm{I}_{\mathrm{p}, \mathrm{l}} \\
{[\mathrm{mm}]}\end{array}$ & $\begin{array}{c}\mathrm{I}_{\mathrm{c}} \\
{[\mathrm{mm}]}\end{array}$ & $\begin{array}{c}\mathrm{I}_{\mathrm{p}, \mathrm{r}} \\
{[\mathrm{mm}]}\end{array}$ & $\begin{array}{c}\mathrm{r} \\
{[\mu \mathrm{m}]}\end{array}$ & $\begin{array}{c}\mathrm{h}_{\mathrm{t}} \\
{[\mu \mathrm{m}]}\end{array}$ \\
\cline { 2 - 11 } & 1.95 & 21.5 & 3 & 0.62 & 10 & 2.5 & 1 & 0.5 & 80 & 0.3 \\
\hline
\end{tabular}

Tableau 3. Geometrical parameters used to model the REF test condition investigated by Bech [4]. In this case, the lubricant escapes both backward (MPHDL) and forward (MPHSL)

The geometrical dimensions used to model the REF test conditions are listed in Tableau 3. The initial length of both pipes is determined from Bech's measurements of the lubricant escapes at the front and the rear of the cavity. The pipe thickness is chosen as $0.3 \mu \mathrm{m}$ : an order of magnitude smaller than the peak-to-valley measurement on the plateaus where relubrication occurs.

Due to the very small thickness used for the pipes, the resulting mesh for the whole model is really too big to solve the problem on a personal computer. Therefore we only consider a small rectangular region of the strip around the pocket. The strip thickness is $1.95 \mathrm{~mm}$ as in Bech's experiments. Its length is $1 \mathrm{~mm}$ larger than the fluid domain: $0.5 \mathrm{~mm}$ in excess on each side.

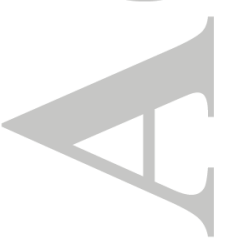

This article is protected by copyright. All rights reserved. 


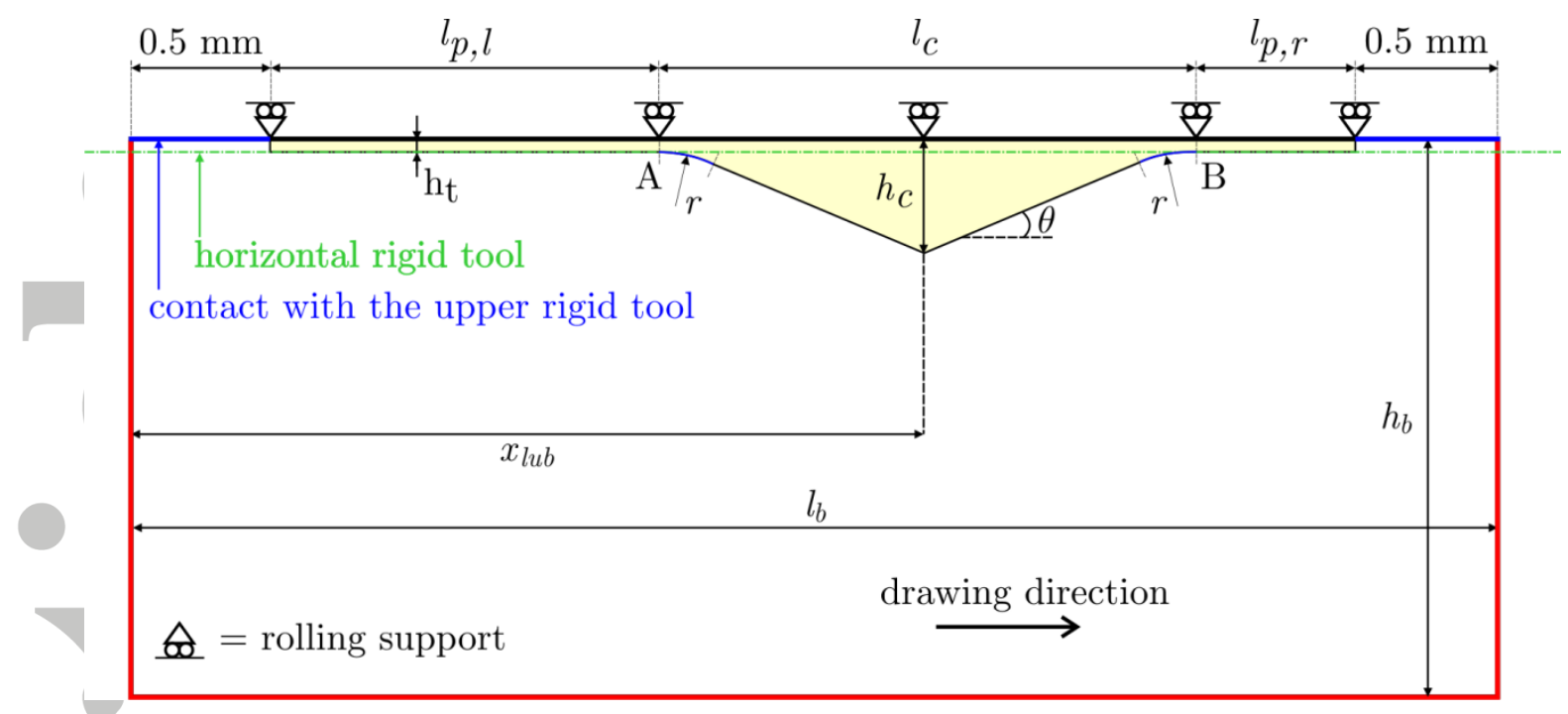

Figure 22. Initial geometry of the reduced model used to observe lubricant escape at the rear or at the front of the pocket in the REF test condition from Bech's experiments.

\subsubsection{Material}

The strip elastoplastic material as well as the viscous fluid behavior are modelled with the material laws as in the full model discussed above.

\subsubsection{Boundary conditions}

\section{Prescribed displacements}

The length of the mesh $\left(I_{b}=5 \mathrm{~mm}\right)$ is smaller than the contact zone $(9.5 \mathrm{~mm}$ in the REF test case see Figure 19). To be representative of the real process, displacements are prescribed on the lower edge and the vertical edges of the mesh. These displacements are approximated from a prior simulation using the first full-scale model (without ALE pipes) described in the previous section. In this way, the effects computed by the microscopic model are representative of the strip drawing process even though the whole strip is not considered.

Practically speaking when analysing the full (macroscopic) model results, two configurations noted 1 and 2 are selected when the lubricant cavity is at the beginning of the contact zone and close to the exit (see Figure 23). They correspond to the initial and final configuration considered in the microscopic simulation.

In the reduced model, to go from the initial geometry to configuration 1, prescribed displacements linearly interpolating these two stages are applied between $t_{0}$ and $t_{1}$ (see Figure 23-b - Phase 1). Then prescribed displacements interpolating the positions recorded in the macroscopic model at each time step are used (see Figure 23-b Phase 2). 
The lubricant escape on the plateaus computed by the reduced model might have an effect at the scale of the full model which would impair the reliability of the present approach. However, we think that effect is negligible. Indeed, the boundary conditions applied on the edges of the reduced model are far from the main cavity and therefore, their effect is therefore limited: the displacements applied on the bottom strip cannot be further away from the cavity since the whole strip thickness is accounted for in the model. The displacements applied on the vertical edges are $1 \mathrm{~mm}$ away from the cavity right edge ( $\mathrm{nb}$ : this is the worst case since the left edge is $3 \mathrm{~mm}$ away from the left part of the cavity) which is large regarding the scale at which the fluid flow occurs. Also, the full model accounts for contact loss between the tool and the strip due to, on the one hand, lubricant pressure in the cavity compared to the solid-to-solid pressure and, on the other hand, the deformation of the cavity (see Figure 20 for instance). This contact loss is somewhat similar to the lubricant escaping from the main cavity. Therefore, we think that the boundary conditions applied to the reduced model would not be much affected by the lubricant escapes.

The computational interval is $12.2 \mathrm{sec}$ which is smaller than in the full scale model since we focus on the time interval where the main cavity is in the contact zone.

\section{Contact management}

The upper edge of the strip is in contact with an additional rigid tool identical to the one used in the macroscopic model. As in the macroscopic model, there is a rigid horizontal tool, at a distance $h_{t}$ from the upper tool which enforces a minimal thickness of the pipe preventing mesh distortions.

Contact conditions are still managed by the penalty method. The same penalty value as in the macroscopic model is used for the upper surface of the strip. A value ten times larger is chosen for the contact with the lower part of the pipes. By doing so, the size of the normal gap on the lower edge of the pipes is reduced preventing any mesh distortions.

\begin{tabular}{|c|l|r|r|}
\hline Contact tool & Entity in contact with the tool & $\begin{array}{c}\mathrm{C}_{\mathrm{N}} \\
{[\mathrm{MPa} / \mathrm{mm}]}\end{array}$ & $\begin{array}{c}\text { Coulomb's friction coefficient } \\
\mu_{\mathrm{C}}\end{array}$ \\
\hline$\# 1$ & top of the strip & $510^{7}$ & 0.05 \\
\hline$\# 3$ & lower part of the cavity & $510^{8}$ & 0.05 \\
\hline
\end{tabular}

Tableau 4. Normal penalty and friction coefficient values used for the different contact interaction. 
a) Stage 1 : full model (without pipes)

- Configuration 1 chosen in the full model

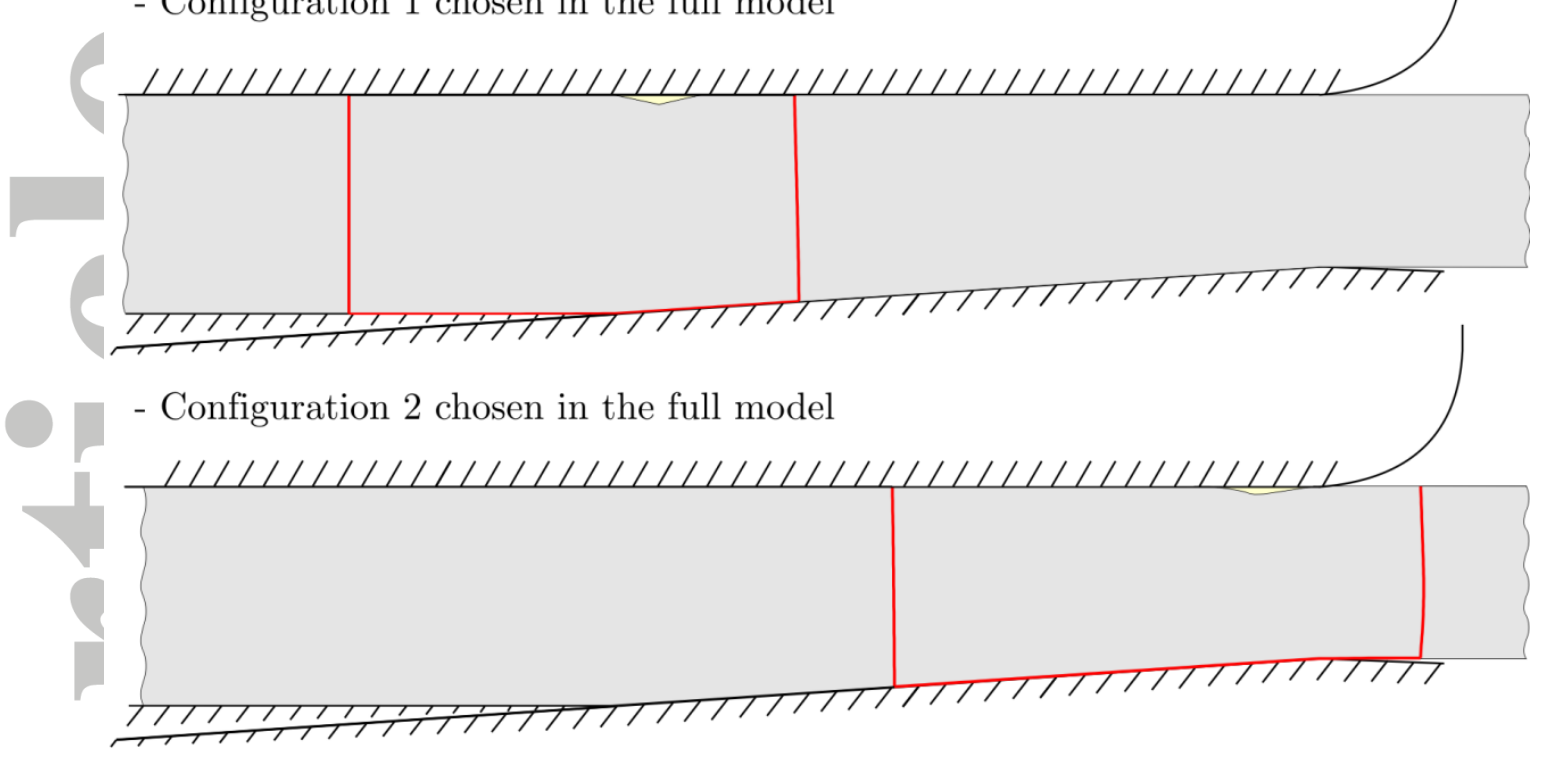

b) Stage 2 : reduced model (with pipes)

- Phase 1 : prescribed displacements applied on the initial geometry to obtain the shape of configuration 1 chosen in the macroscopic model

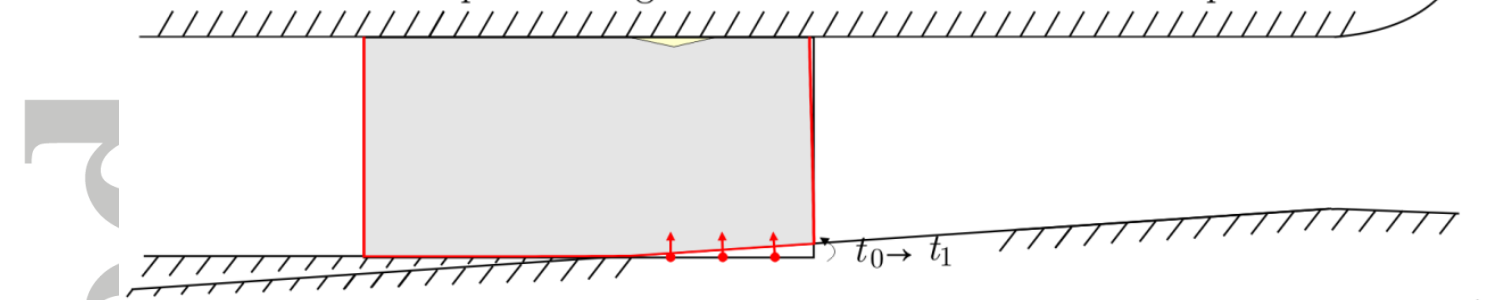

- Phase 2 : prescribed displacements applied on the edges of the microscopic model (except from the upper edge)

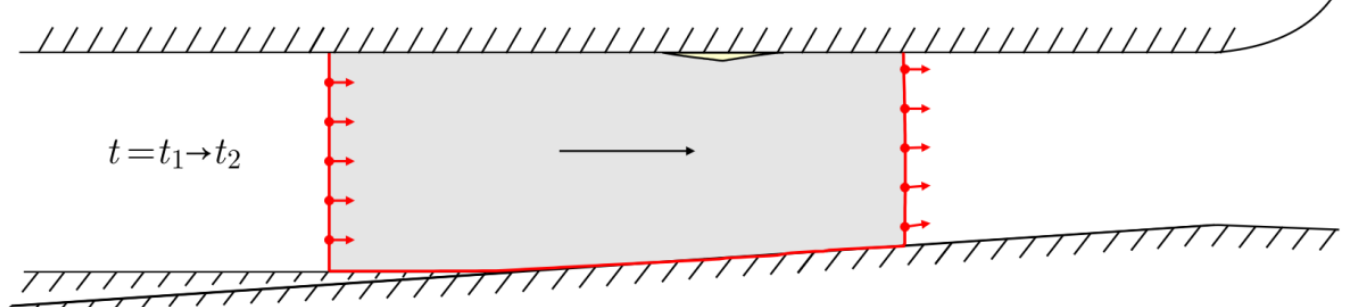

- End of the simulation $t=t_{2}$

11111111111111111111111111111111111111111111

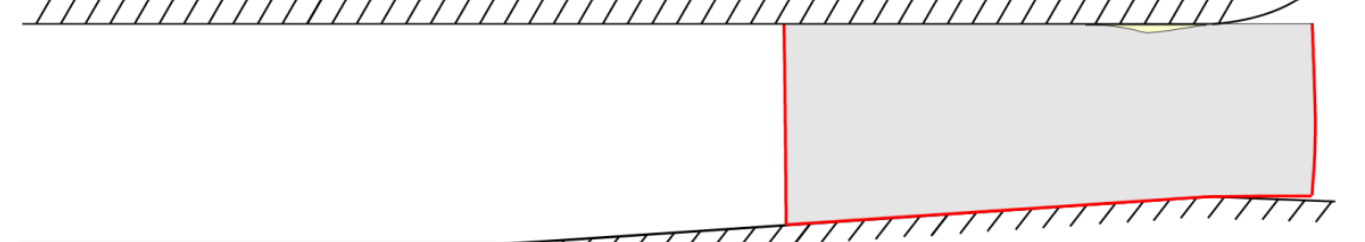

Figure 23. Schematic view of the methodology used to apply prescribed displacements on the edges of the reduced model. A) Selection, from the full-model results, of the initial and final configuration to be used in the reduced model. B) Application of the prescribed displacements in two phases. Phase1: displacement applied to match the shape of configuration 1 chosen in the full model. Phase 2: prescribed displacements linearly interpolating the successive positions of the edges computed in the full model. 


\section{Boundary conditions applied on the upper part of the fluid}

For the same reasons as in the full model, the vertical motion of the fluid free surface is prevented by rolling support conditions. However, in this case, the fluid adhesion to the tool must be taken into account since lubricant escapes at the rear are affected by the hydrodynamic effect in the converging gap between the tool and the cavity.

Initially, adhesion conditions ( $V_{x, f}=0$ - the upper tool is fixed) are applied on the nodes at the top of the triangular cavity while the nodes in the pipes are free to move horizontally (see Figure 24-a. Indeed, the lubricant initially located in the pipes is a numerical trick which allows fluid motion initiation on the plateaus.

Adhesion conditions are automatically updated at given times during the simulation depending on the local lubricant film thickness in the pipes. Once the local thickness is larger than twice $h_{t}$, adhesion is enforced. However, when the local thickness falls below this threshold this condition is deactivated and the nodes are again free to move horizontally. In this way the amount of oil drawn on the plateaus once the pocket is closed is reduced.

In the simulation presented here, the automatic update occurs each time the strip moves $0.25 \mathrm{~mm}$ in the contact zone.

a) Initial configuration :

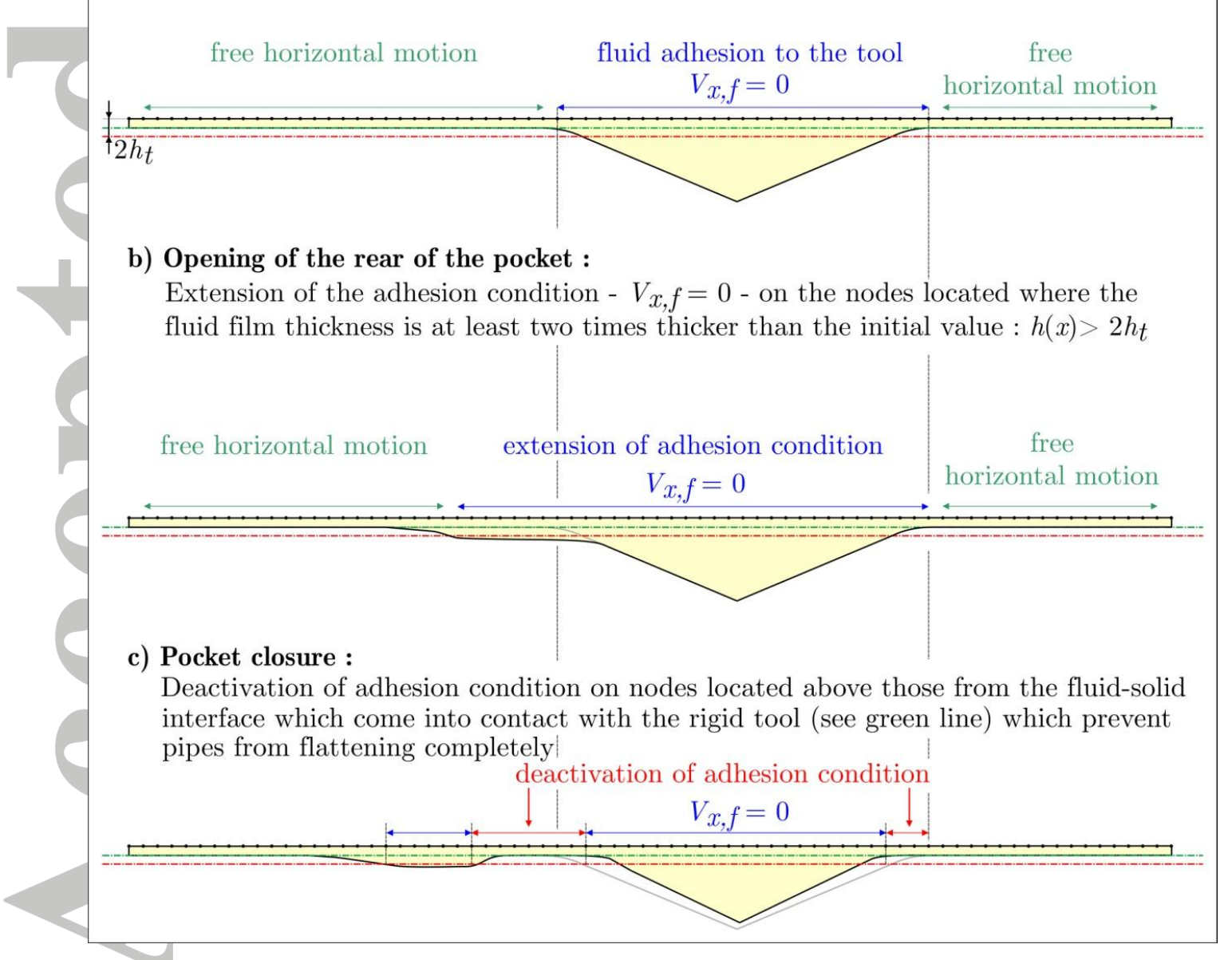

Figure 24. Schematic representation of the evolution of boundary conditions applied on the upper part of the fluid domain in the reduced model.

This article is protected by copyright. All rights reserved. 


\subsubsection{Mesh}

The mesh used to model the REF test condition is shown in Figure 25-a.

Four elements are used through the cavity thickness. This is the minimal number of elements to achieve a sufficient precision in the hydrodynamic pressure computation in the converging gap, at the rear of the pocket. Thus, 280 elements are generated on the cavity width. Their size follows a geometrical distribution to keep an aspect ratio close to 1 on the whole domain (see Figure 25-c and d). To do so, the ratio between the largest and smallest element is 115 .

To reduce the number of degrees of freedom, only two elements are applied on the pipe thickness. Since these elements are only intended to receive and transport the lubricant that escapes from the cavity, a finer mesh is unnecessary. The element length varies linearly over $2 \%$ of the length of the pipe and then remains constant (see Figure 25-e). The maximum length of these elements is 20 times the initial thickness $h_{t}$.

The transition from the mesh of the pocket and the one of the pipes is managed with an unstructured mesh generator. The transition zone at the rear of the cavity is shown in Figure 25-f. The total number of fluid elements is 2430 .

To minimise the distortion of the fluid elements, the ALE formalism is used. At the end of each time step, the nodes located on the upper horizontal edge of the fluid mesh are repositioned opposite to the nodes of the fluid-solid interface. The nodes located at the centre of the transition zone (see Figure 24-f blue frame) are repositioned with the Giuliani's smoothing method [55]. The rest of the inner fluid nodes are obtained by a simple interpolation using the transfinite mesher.

The solid part is discretised with 10583 elements. The lower part of the sheet is meshed with $0.05 \times$ $0.05 \mathrm{~mm}$ quadrangles, as in the full model, on $80 \%$ of the total height. On the remaining $20 \%$, the sheet metal elements are generated using an unstructured mesh generator to manage easily the transition between the fluid domain and the regular mesh of the sheet. Contrarily to the fluid part, the mesh of the solid portion is purely Lagrangian.

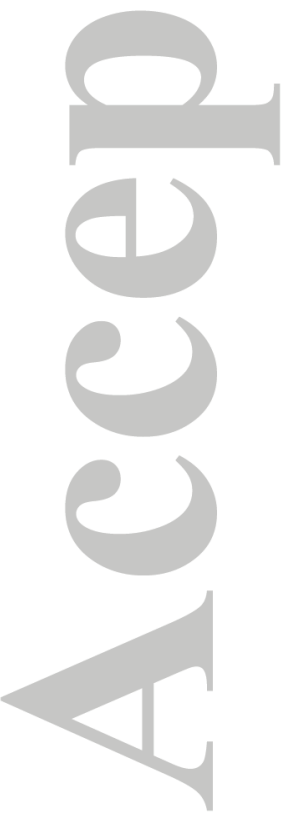

This article is protected by copyright. All rights reserved. 
a) Overview of the mesh (the fluid part is depicted in yellow)

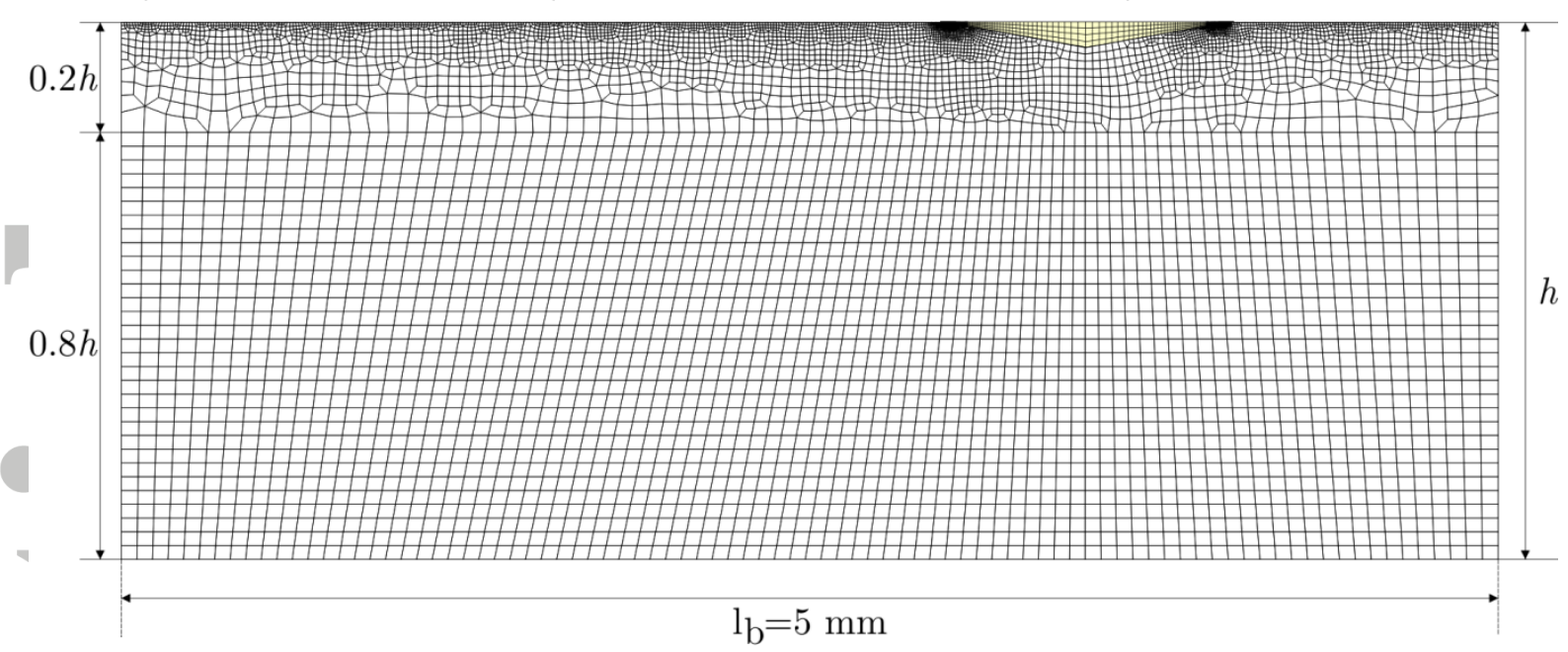

b) Mesh of the lubricant cavity and the pipes

$$
\mathrm{l}_{\mathrm{t}, \mathrm{l}}=2.5 \mathrm{~mm} \quad \sim \mathrm{l}_{\mathrm{p}}=1 \mathrm{~mm} \quad \mathrm{u}^{\mathrm{l}_{\mathrm{t}, \mathrm{r}}=0.5 \mathrm{~mm}}
$$

c) Mesh of the lubricant pocket

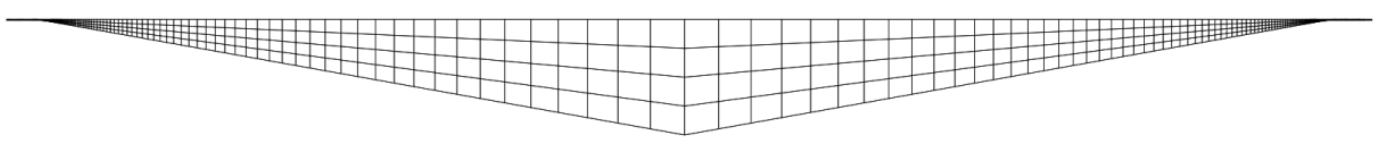

d) Zoom on the rear part of the pocket

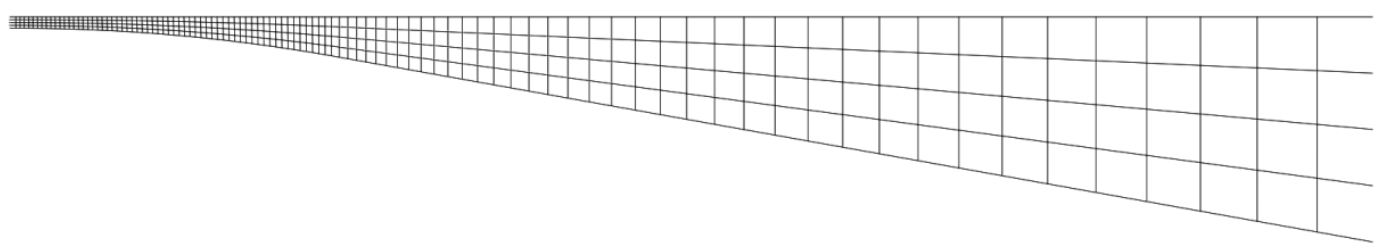

e) Transition between the cavity and the pipe $(4 \rightarrow 2$ elements $)$

f) Zoom on the mesh transition : $4 \rightarrow 2$ elements on the thickness of the pipe

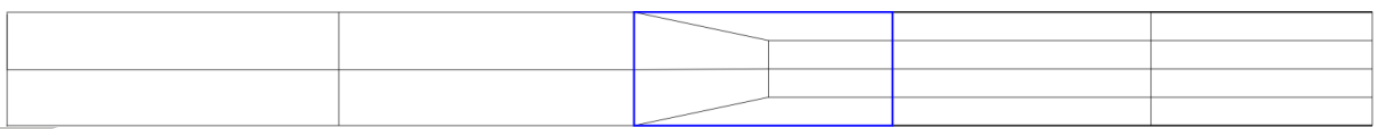

Figure 25. a) Mesh used in the reduced model. It is made of 10583 solid elements and 2430 fluid elements. b) Mesh of the fluid part c) - d) -e) -f) Successive zoom on the fluid elements located at the rear of the cavity.

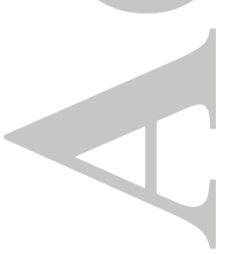




\subsubsection{REF test condition: results analysis}

In the REF test condition, Bech [4] observed lubricant escape at the rear and at the front of the main lubricant cavity. For this case, as we shall see, the numerical model also predicts two openings of the cavity at the rear and at the front, therefore generating micro-cavities on the plateaus.

The evolution of the shape of the upper part of the lubricant cavity and the lower part of the pipes during strip drawing is depicted in Figure 26, Figure 27 and Figure 28. Initially, the two pipes are in contact with the horizontal tool enforcing a minimal film thickness $h_{t}$ (see Figure $26-a$ ).

In Figure 26-b the rear part of the cavity opens allowing some lubricant to flow on the plateaus at the rear. Then, the cavity closes generating a micro-cavity on the plateaus. This cavity remains stationary due to adhesion condition (see Figure 26-c, $d$ and e) until it reaches the end of the pipe (see Figure 26-f) which is a limitation of the present model.

In the meantime, the triangular cavity opens at the front (see Figure 27-d) generating a micro-cavity (see Figure 27-e). This micro-cavity then resorbs due to the pressure induced by the strip deformation in this area and the permanent link with the main cavity. This micro-cavity then completely disappears: the lubricant which escaped at the front of the cavity is sent back to the main cavity as the bottom of the micro-cavity is moving up due to the strip deformation (see Figure 27-f).

Towards the end of the process, both the rear part and the front part of the main cavity opens (see Figure 27-g and $\mathrm{h}$ respectively). This induces a second micro-cavity on the plateaus at the rear while the front part of the main cavity remains wide open until the end of the contact zone is reached. At the end of the simulation, the opening at the front $(6 \mu \mathrm{m})$ is much larger than the depth of the micro-cavities at the rear $(3 \mu \mathrm{m})$.

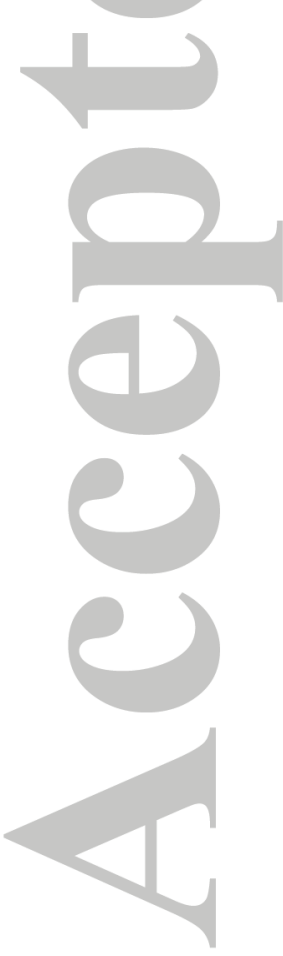

This article is protected by copyright. All rights reserved. 
a) Initial configuration

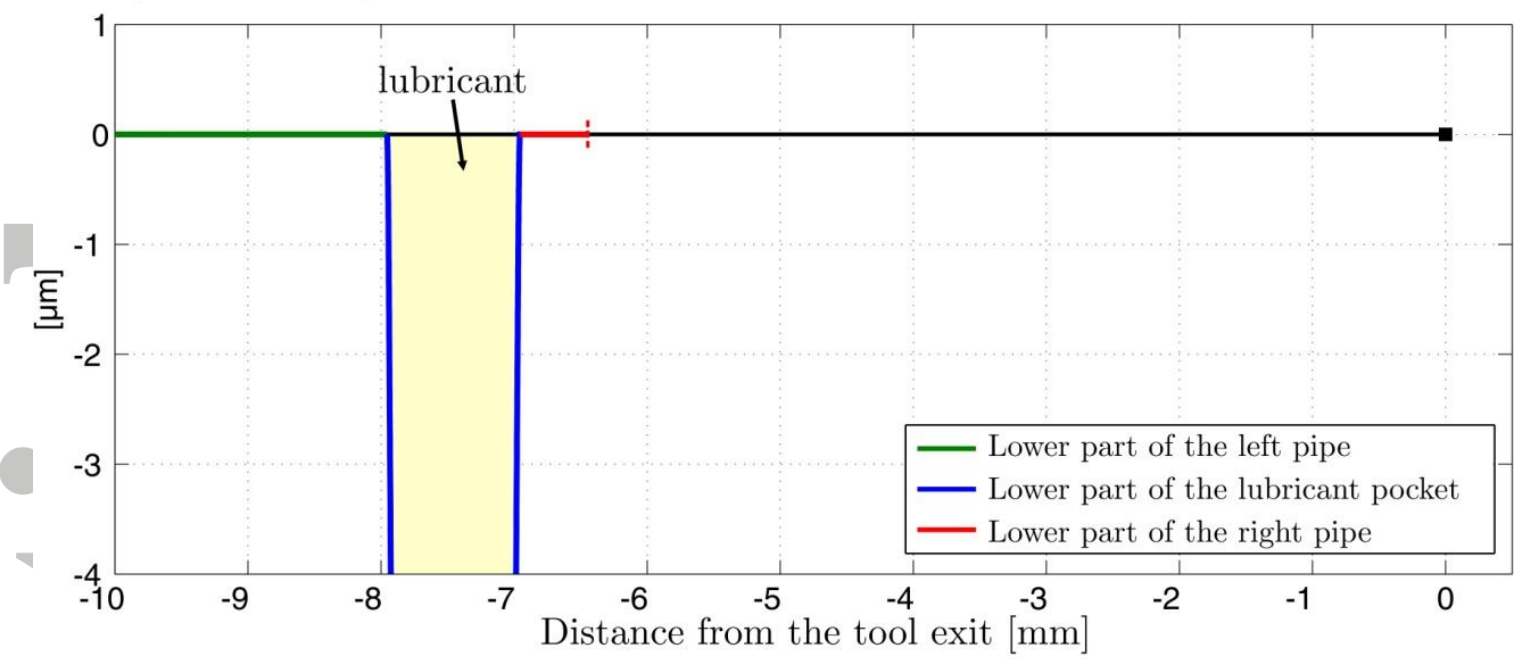

b) Opening of the rear of the pocket (MPHDL)

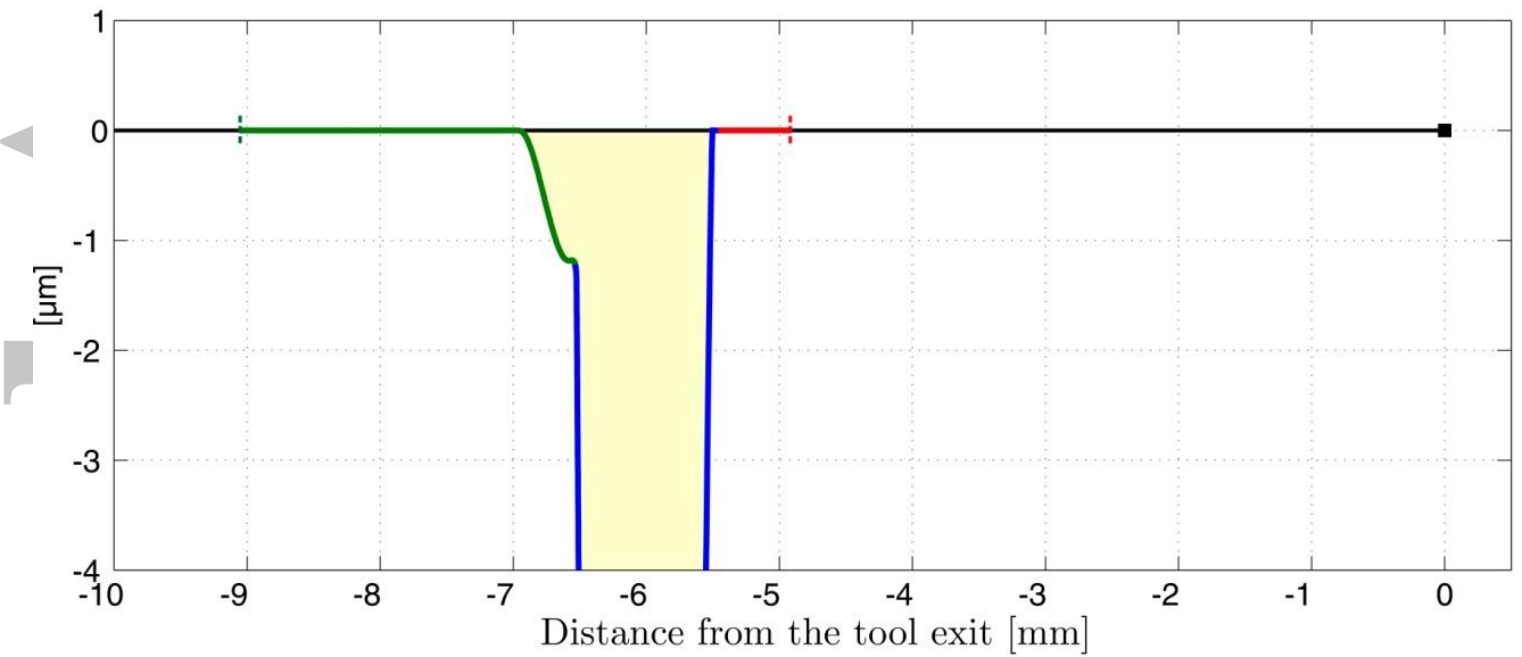

c) Closing of the rear of the pocket

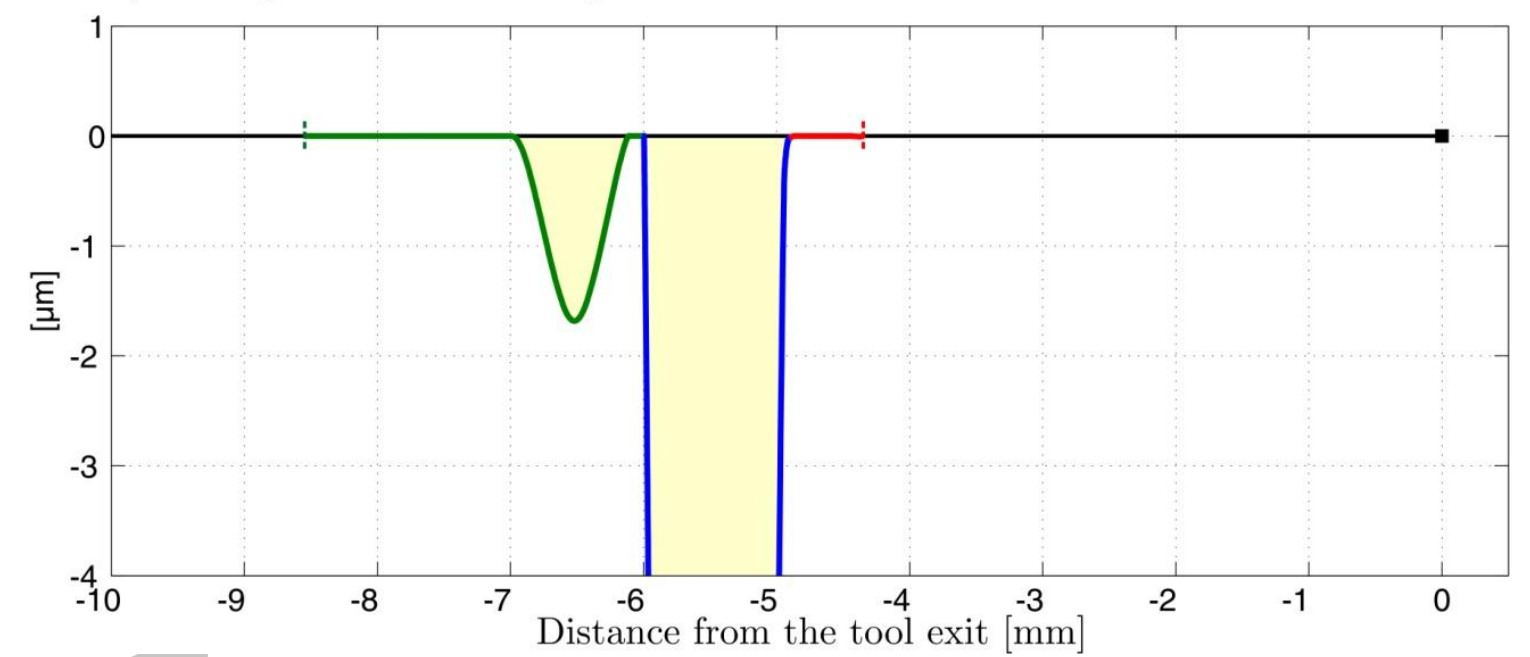

Figure 26. Evolution of the shape of the lower part of the lubricant pocket and the pipes for successive positions in the contact zone: REF test condition (part 1/3). The vertical scale has been magnified by a factor of 1000 . 
d) Opening of the pocket front (MPHSL)

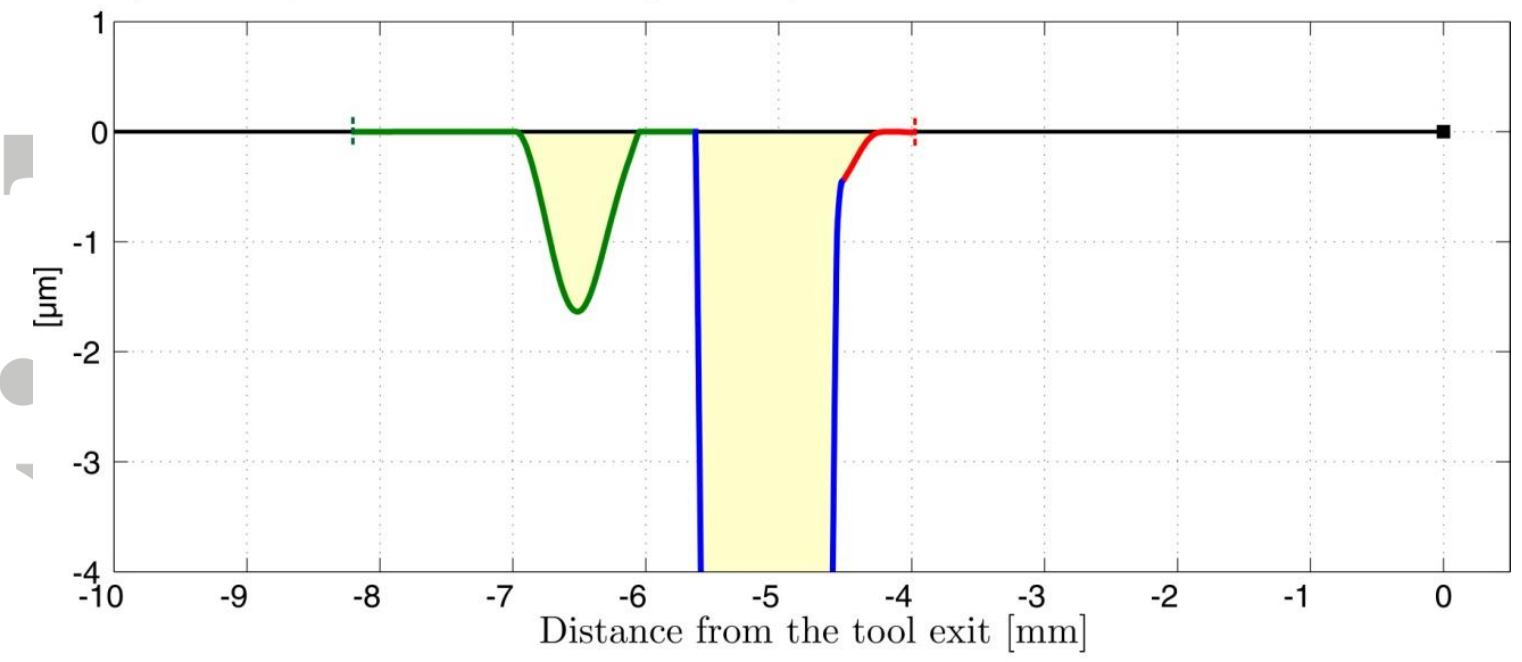

e) Closing of the pocket front

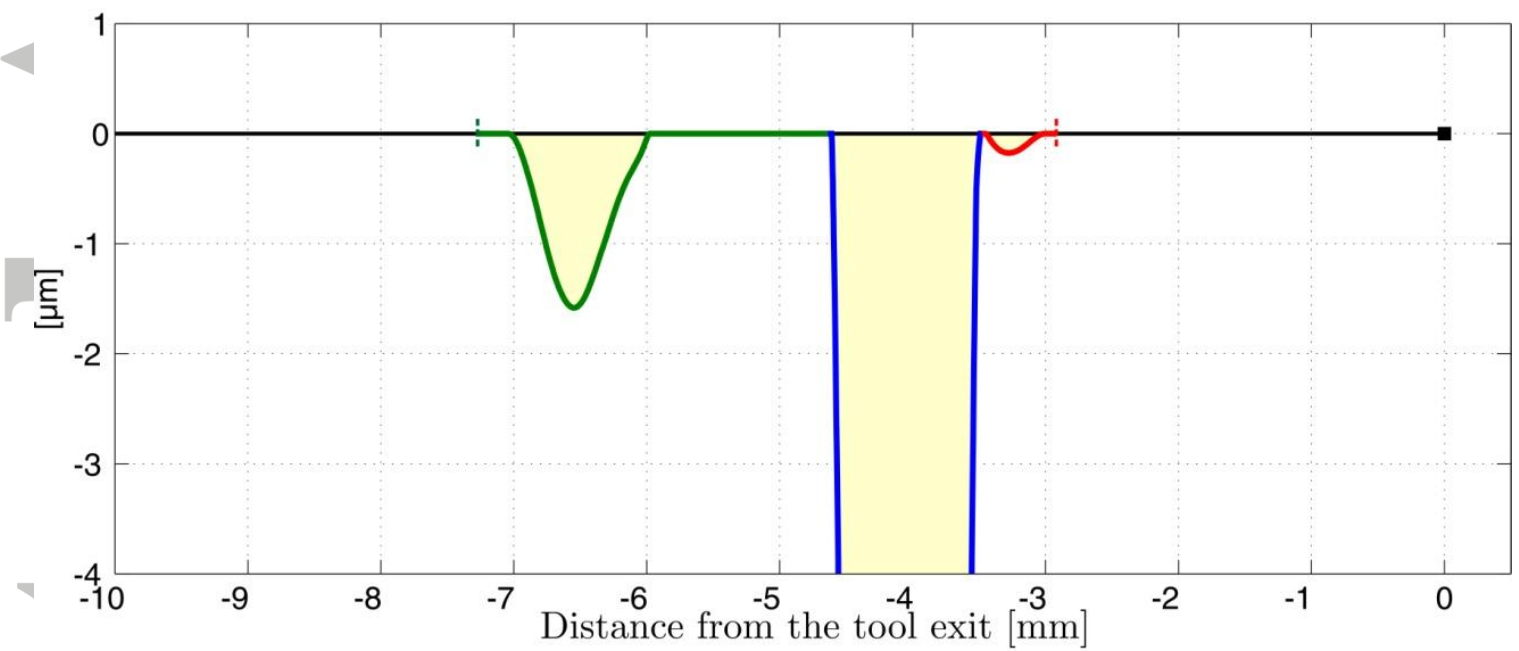

f) The micro-cavity generated at the rear reaches the end of the left pipe resorption de la micro-cavity located at the front

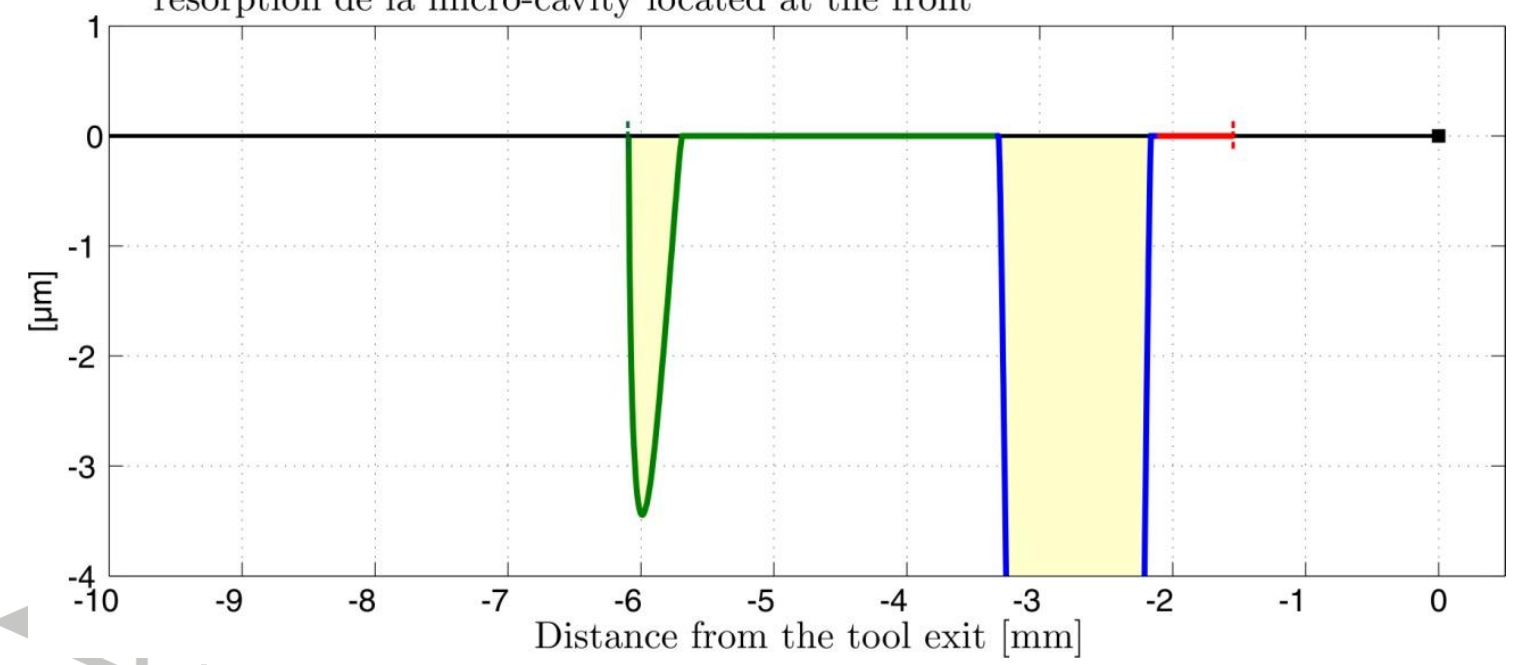

Figure 27. Evolution of the shape of the lower part of the lubricant pocket and the pipes for successive positions in the contact zone : REF test condition (part 2/3). The vertical scale has been magnified by a factor of 1000 . 
g) Compression of the fluid located at the frond of pocket

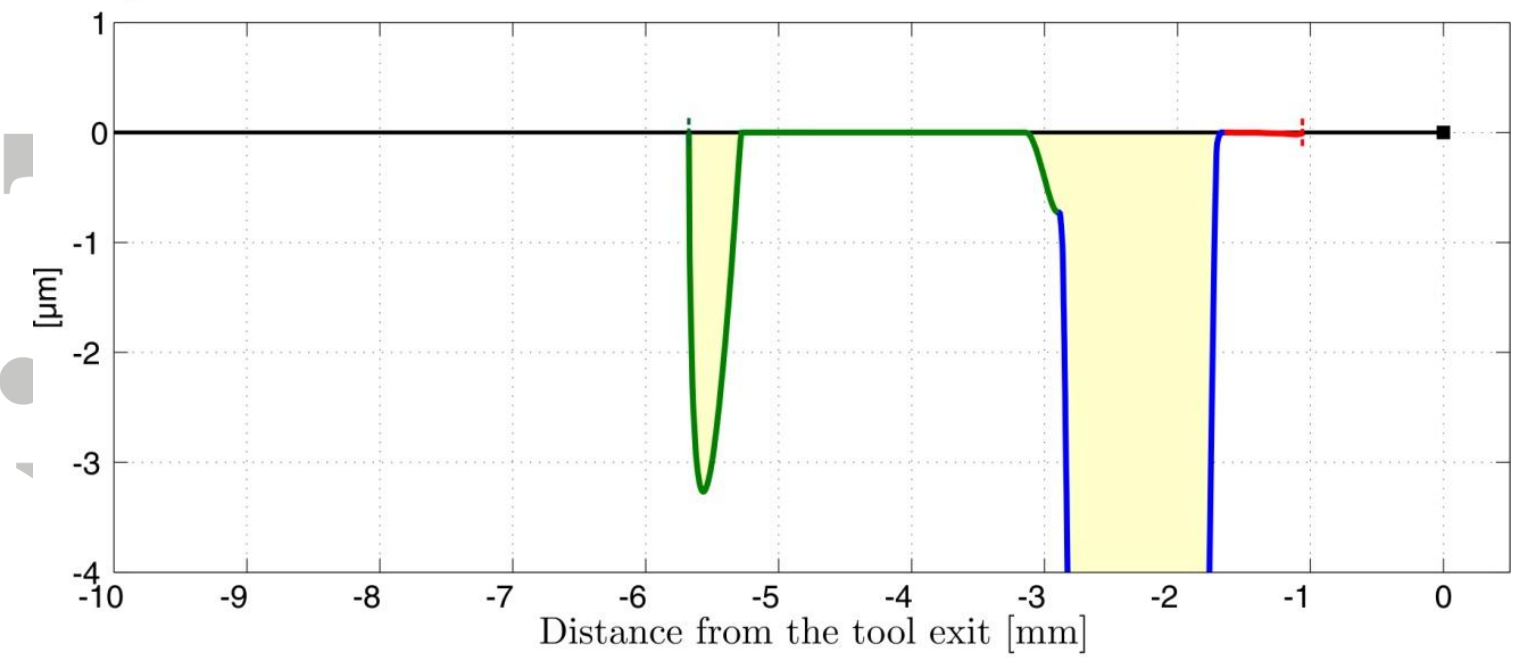

h) 2nd opening of the rear (MPHDL) and the front (MPHSL) of the pocket

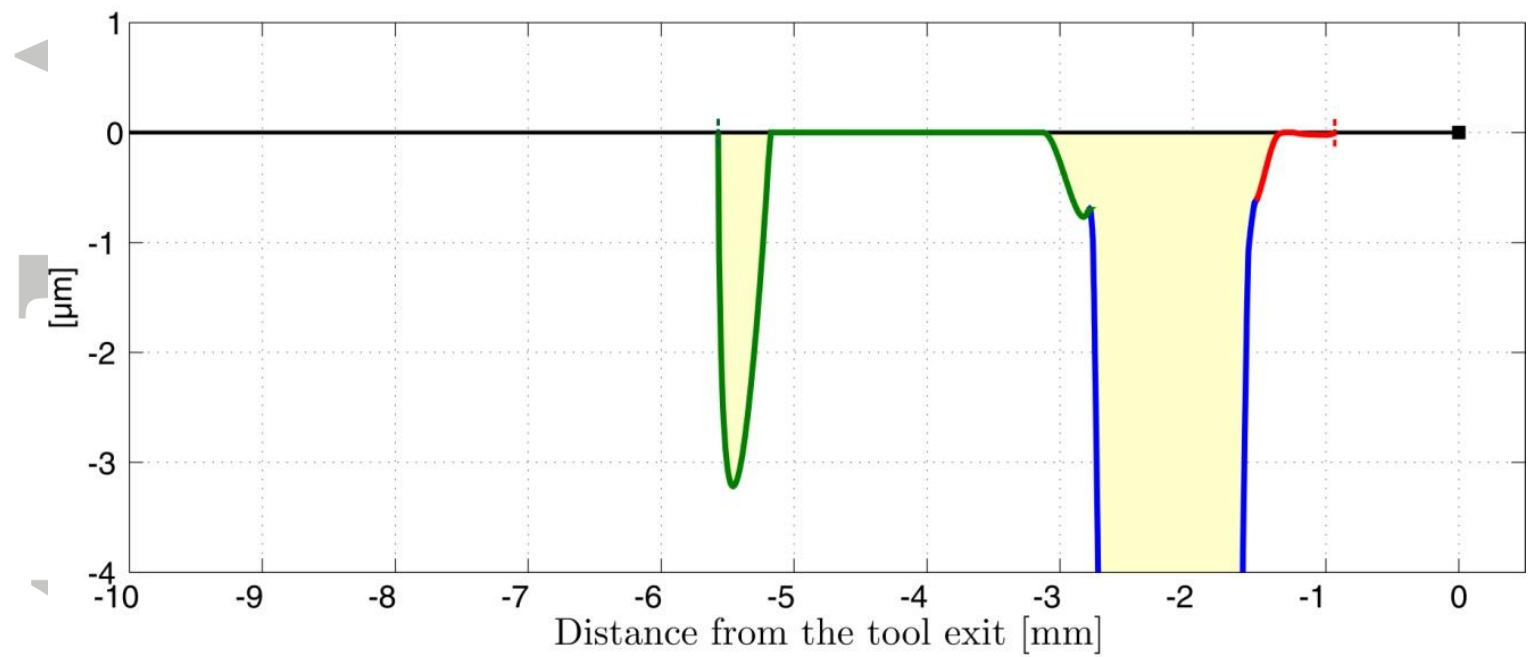

i) Closing of the rear of the pocket - opening of the pocket front (MPHSL)

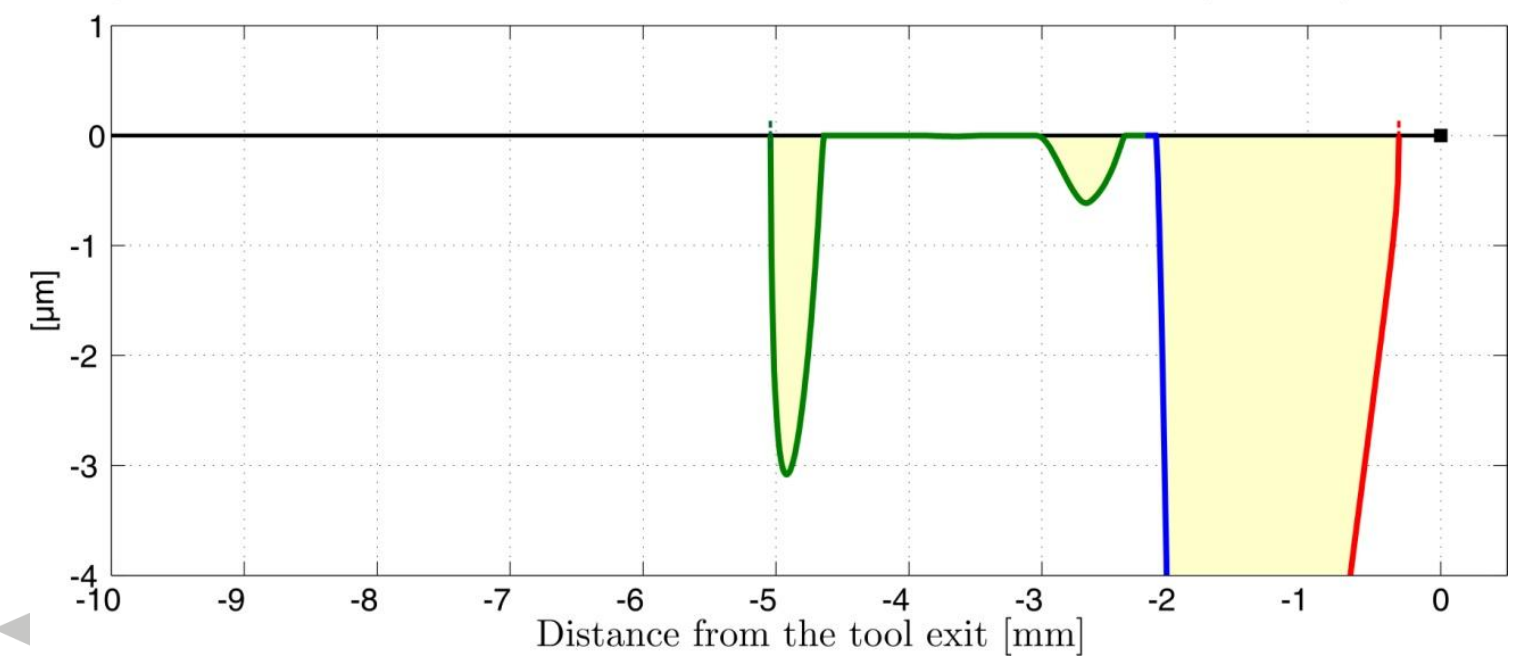

Figure 28. Evolution of the shape of the lower part of the lubricant pocket and the pipes for successive positions in the contact zone: REF test condition (part 3/3). The vertical scale has been magnified by a factor of 1000 . 


\section{Cavity closure}

The evolution of the shape of the lubricant pocket and the pipe discussed above and depicted in Figure 26, Figure 27 and Figure 28 shows that the rear and front parts of the lubricant pocket close once some lubricant has escaped (see for instance Figure 26-c, Figure 27-e and Figure 28-i). This observation seems to be consistent with experiments.

Indeed, two consecutive snapshots of a video recorded by Bech [4] during one of his tests, represented in Figure 29, show that the rear of the pocket closes once lubricant has escaped backward and a solid-to-solid contact zone between the tool and the strip appears. The fluid is still present on the plateaus, but the adhesion of the fluid to the upper tool tends to pull the fluid away from the main cavity. According to these experimental observations, the pocket closure predicted by the numerical model seems to have a physical meaning.
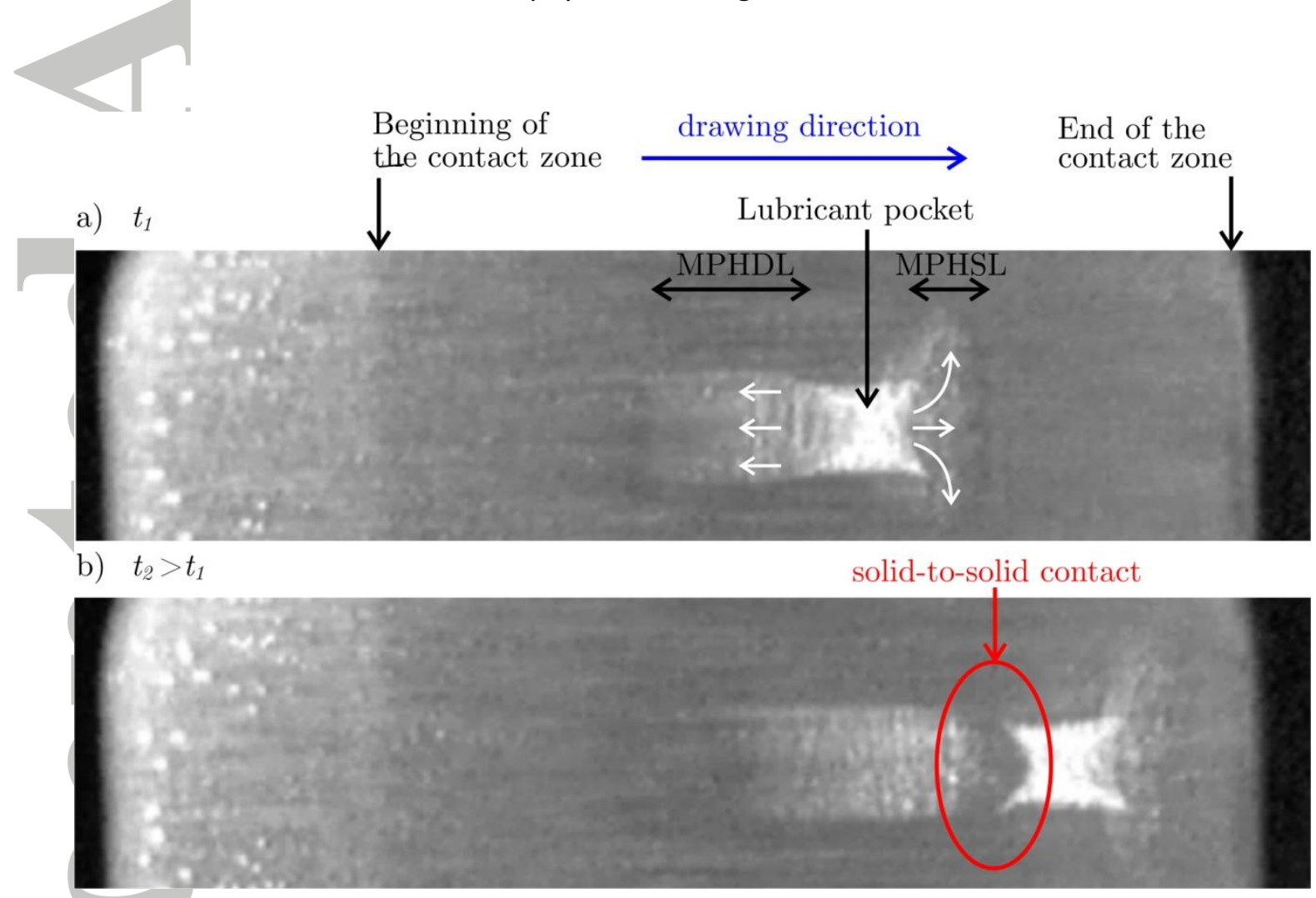

Figure 29. Snapshots taken from a video recorded by Bech during one of his experiments. These pictures show the rear of the pocket closes once MPHDL has occurred.

This article is protected by copyright. All rights reserved. 


\section{Influence of the initial lubricant film thickness on the plateaus}

Since it can be considered as a pure artifact, the effect of the initial pipe thickness $\left(h_{t}\right)$ is investigated. Simulations are conducted with $h_{t}=0.3-0.4$ and $0.5 \mu \mathrm{m}$. This section shows that the value of $h_{t}$ has minimal influence on the simulation results proving the legitimacy of the current approach.

Figure 30 represents the vertical displacement of the geometrical point situated at the rear of the cavity (see point a, at the transition between the fillet and the left pipe, in Figure 22) for the three values of $h_{t}$ mentioned above. The three resulting curves overlap nearly perfectly. In all cases, there are two openings of the back of the pocket in accordance with the analysis carried out previously. The first pocket opening starts $7.1 \mathrm{~mm}$ ahead of the tool exit while the second one begins at $3.2 \mathrm{~mm}$ from the exit. The maximum displacement is more important for the first opening $(1.32 \mu \mathrm{m})$ than for the second one $(0.81 \mu \mathrm{m})$.

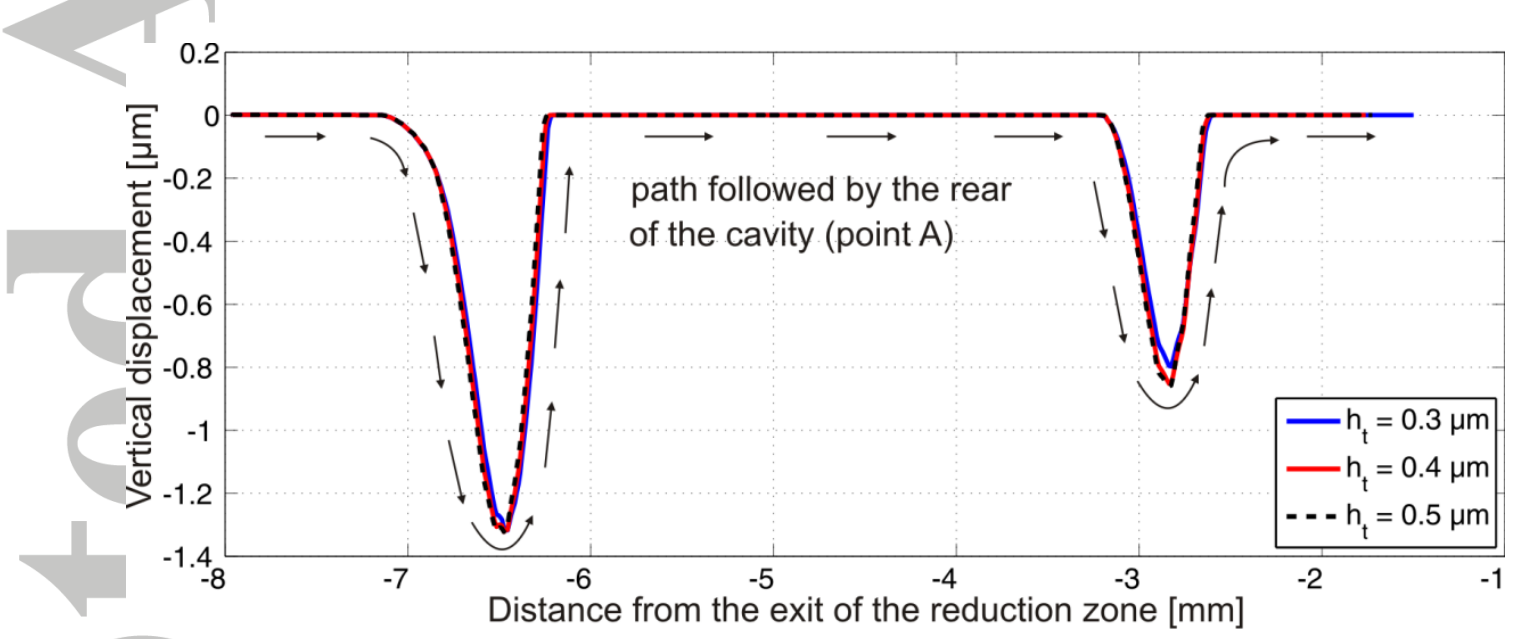

Figure 30. Vertical displacement of the rear part of the cavity (see point A in Figure 22), while the strip is pulled through the reduction zone, for three initial pipes thickness $\left(h_{t}\right)$ values. The arrows refer to direction of the motion.

The vertical displacement of the front of the pocket is shown in Figure 31. Again, the three curves obtained with different values of $h_{t}$ used perfectly overlap. Two cavity openings take place. They respectively start at 4.9 and $1.6 \mathrm{~mm}$ of the exit of the contact area. This time, the maximum displacement is larger for the second opening $(6.6 \mu \mathrm{m})$ compared to the first one $(0.9 \mu \mathrm{m})$.

This article is protected by copyright. All rights reserved. 


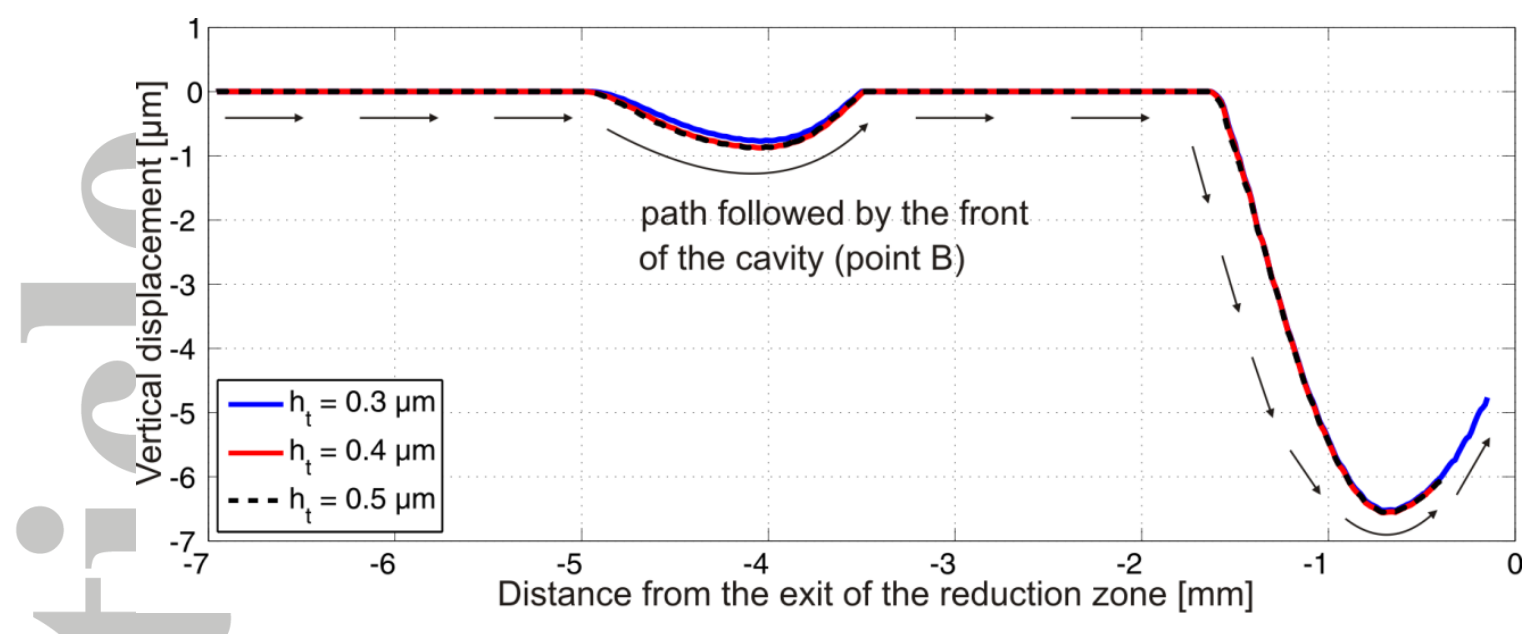

Figure 31. Vertical displacement of the front part of the cavity (see point B in Figure 22), while the strip is pulled through the reduction zone, for three initial pipes thickness $\left(h_{t}\right)$ values. The arrows refer to direction of the motion.

The value of $h_{t}$ does not influence the cavity openings but it has an influence on the amount of lubricant dragged on the plateaus: the larger the initial film thickness, the larger the pipe volume variation.

The evolution of the volume brought to the left pipe as the strip is drawn through the tool is represented in Figure 32. The trends are similar for the three values of $h_{t}$ investigated here. First, there is a significant volume change when the rear of the pocket opens. Then, between 4 and 7.5 seconds, while the rear of the cavity is closed, the volume increases linearly: the larger $h_{t}$, the larger the slope. This is due to the permanent link between the micro-cavity generated on the plateaus and the main cavity. Indeed, the adhesion condition applied on the fluid in the micro-cavity brings more lubricant from the main cavity into the micro-cavity as the strip is pulled through the tool.

When $t=8$ seconds, a reduction in volume is observed. This is due to the second opening of the pocket which draws some fluid out of the pipe. At $t=9$ seconds, there is a large volume variation due to the closure of the rear of the pocket which again brings some lubricant on the plateaus. Finally, the volume increases linearly for the same reasons as explained above.

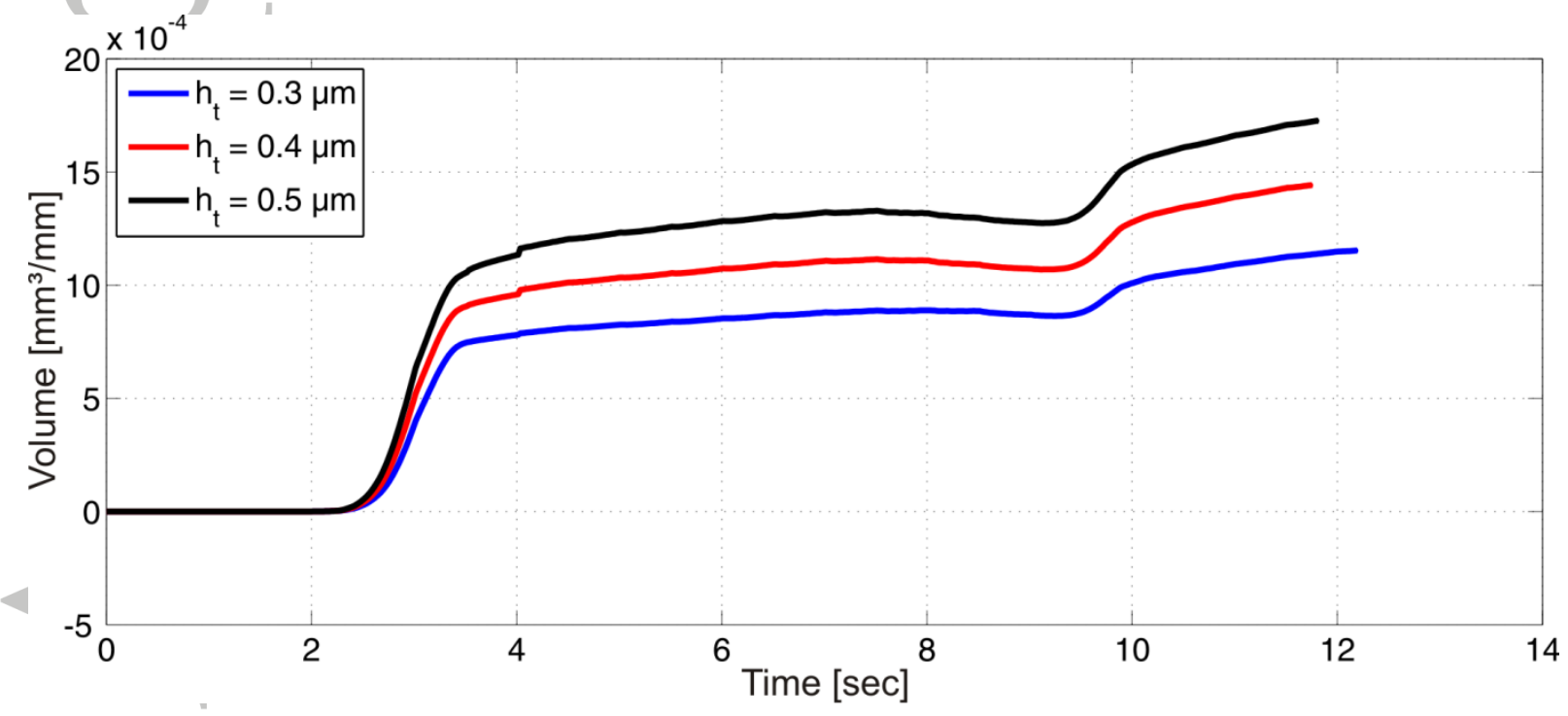


Figure 32. Volume brought to the left pipe when modelling REF test condition from Bech's experiments for three initial pipe thickness values. The larger $h_{t}$, the larger the volume.

The results discussed above show the model is able to reproduce plane strip drawing experiments highlighting microscopic lubricant flows. The obtained results demonstrate the model is able to predict the Micro-Plasto-HydroDynamic lubrication at the rear of the pocket as well as the MicroPlasto-Hydrostatic flow at the front of the cavity.

Although this model is much more representative than those described in the literature, it still presents, like any model, different limitations:

- Numerical simulations described here are conducted in 2D assuming plane strain conditions. Therefore, the cavity has a constant triangular section along the width of the strip whereas the actual shape is pyramidal. The present model therefore neglects 3D effects such as flows in the transverse direction (see A1. - B5 - C5 - C6 - B7 - C7 in Figure 16). However, the extent of the lubricant flow in the transverse direction is generally much smaller than the one in the drawing direction.

- The piezo-viscous behaviour of the lubricant is not taken into account. To consider this, a precise characterisation of the lubricant is needed. Since we do not have accurate data for the lubricant used by Bech [4] during his tests, this dependence is not accounted for in the present work.

- The micro-cavities formed on the pipes are permanently connected to the main cavity through the pipes. Therefore, a fluid motion between the main cavity and micro-cavities on the plateaus is possible while the main cavity is closed in theory. This comment is mitigated by the fact that some micro-roughness is present on the strip and tool surfaces. The pipes in our model can be seen as longitudinal micro-roughness channels with a constant minimal thickness.

- Pipes used in the model have a finite length fixed a priori. Therefore, in order to predict the mark of the lubricant left by the fluid flowing on the plateaus, initial pipe lengths have to be sufficiently large.

- The equations solved in the model are based on the assumption of continuous medium. This assumption is somewhat undermined given the thickness of the pipes used in the simulations $(0.3 \mu \mathrm{m})$, which approaches the size of the metal grains.

- The present model is not able to represent the roughness increase observed by Bech in his tests. When a full film separates a tool and a metal sheet deforming plastically, the metal grains located close to the surface of the sheet can slide relative to each other which generate an increase in the strip roughness. This effect, called roughening, has an influence on the lubricant flow on the plateaus and is not taken into account in the present model.

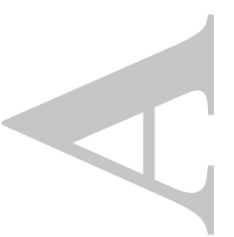

This article is protected by copyright. All rights reserved. 


\section{Conclusion}

This paper presents a multi-scale finite element model capable of modelling the occurrence of micro-plasto-hydrodynamic (MPH) lubrication. This phenomenon consists in lubricant flows from isolated surface cavities and has been observed in various sheet metal forming processes. For instance, several authors such as Bech witnessed lubricant being squeezed out from lubricant oil pockets at the front and the rear during plane strip drawing.

Modelling MPH lubrication requires a numerical tool capable of performing fluid-structure interaction simulations. The simulations presented here are conducted with Metafor, an in-house finite element code initially developed to model sheet metal forming simulations. A Norton-Hoff material law is implemented to model the behaviour of a Newtonian fluid and several tests cases of increasing complexity are run in order to prove the reliability of the software results when studying fluid flows and fluid-structure interaction configurations.

The difficulties linked to large deformations of low viscosity fluid elements are overcome thanks to the use of the Arbitrary Lagrangian Eulerian formalism, which allows us to uncouple the motion of the mesh and the material. Therefore a good quality mesh is achieved throughout the whole fluid domain in all the simulations presented in this paper.

The reliability of the FE results being proved, a plane strip drawing model aiming at reproducing Bech's experimental observations is set up. One of the biggest challenges to tackle when modelling this phenomenon is the microscopic thickness of the flow. To overcome this difficulty, a multi-scale approach is developed. It implies running successively two numerical simulations. The first one models the interaction between the elastoplastic deformation of a strip and the deformation of a triangular cavity filled with lubricant. Displacements recorded in this simulation are then used as boundary conditions in a second model focusing on a smaller area around the lubricant cavity. In this model, two very thin fluid area called pipes are added at both ends of the cavity. Thanks to the use of the ALE formalism, the lubricant can - depending on the current conditions - inflate the pipes and flow on the plateaus.

The model is used to reproduce a test condition encountered by Bech for which he observed a lubricant flow at the rear and at the front of the cavity. The numerical model predicts several openings of the cavity at the rear and at the front allowing lubricant to escape and generating microcavities on the plateaus. This proves that the model is able to predict the Micro-PlastoHydroDynamic lubrication at the rear of the pocket as well as the Micro-Plasto-HydroStatic flow at the front.

The numbers of openings of the main cavity at the rear and at the front as well as the displacement amplitudes are insensitive to the initial pipe thickness $h_{t}$ which justifies the present approach. However, this parameter affects the amount of lubricant flowing on the plateaus when MPHDL and MPHSL occur. Indeed, there is a direct link between these micro-cavities and the main cavity even when the main cavity is closed in theory. Therefore, the adhesion condition applied on the fluid in the micro-cavity tends to bring more lubricant to the plateaus while the main cavity is closed which is a limitation of the model.

The present model is the first of his kind able to accurately predict the onset of MPH lubrication flow and to give an estimate of the local lubricant film thickness.

This article is protected by copyright. All rights reserved. 
The fact that lubricant escapes might occur in several stages is a new feature of MPH flow. This could be confirmed or contradicted by conducting experimental film thickness measurements. These would indeed be helpful to determine whether lubricant film on the plateaus is continuous or not. Such measurements could be performed using ultrasonic sensors. This technique has indeed been used in the field of tribology to measure continuous thin lubricant layers in a number of studies, such as those looking into journal bearings and mechanical seals $[56,57,58,59]$.

The MPH model could be improved by including a more complex behaviour law to model the lubricant. To do so, more experimental data are necessary. Ideally, lubricant viscosity and bulk modulus pressure dependence should be known. This effect could influence the MPHDL onset at the rear of the pocket.

The numerical simulations currently require a long computational time: about 10 days. This is due to small mesh elements in the thin lubricant layers at both ends of the main cavity. These thin lubricant layers could be replaced by contact elements able to determine a local friction coefficient upon the integration of an average Reynolds equation. The latter would take into account the influence of asperities on the flow. Elements of this type have been used by Boman and Ponthot [13] in FE simulations of cold rolling when the hydrodynamic regime occurs in the roll bite. Several challenges will be faced when using such kind of elements. The first one concerns the transition between the solid - solid contact (managed by a conventional Coulomb contact law) to the lubricated contact once the conditions required for the appearance of MPHSL or MPHDL are met. Another difficulty will be to determine the initial lubricant film thickness required to integrate Reynolds equation in order to compute local shear stress. Apart from a smaller computational time, these elements would provide a way to predict the length of the plateaus affected by MPH flows.

Now that the modelling of MPH lubrication has been handled successfully in plane strip drawing, we are now working on the implementation of a cold rolling model capable of prediction the occurrence of this phenomenon.

\section{Acknowledgements}

Yves Carretta gratefully thanks ArcelorMittal, FRIA (Fonds pour la formation à la Recherche dans I'Industrie et dans l'Agriculture), the Walloon Region and the European Social Fund for financial support through grant First-International: convention $n^{\circ} 1217863$.

\section{References}

[1] Mizuno T, Okamoto M. Effects of Lubricant Viscosity at Pressure and Sliding Velocity on Lubricating Conditions in the Compression-Friction Test on Sheet Metals. Journal of Lubrication Technology 1982; 104: 53-59.

[2] Azushima A, Tsubouchi M, Kudo H. Direct observation of lubricant behaviour under the MicroPHL at the interface between workpiece and die. Proc. $3^{\text {rd }}$ Int. Conf. Technol. Plasticity 1990; 1:551556. 
[3] Azushima A. Direct observation of contact behaviour to interpret the pressure dependence of the coefficient of friction in sheet metal forming. Ann. CIRP 1995; 47/1: 479-482.

[4] Bech J, Bay N, Eriksen M. Entrapment and escape of liquid lubricant in metal forming. Wear 1999; 232 (2): 134 - 139.

[5] Sørensen C, Bech J, Andreasen J, Bay N, Engel U, Neudecker T. A Basic Study of the Influence of Surface Topography on Mechanisms of Liquid Lubrication in Metal Forming. CIRP Annals Manufacturing Technology. 1999; 48(1): 203 - 208.

[6] Shimizu I, Andreasen J, Bech J, Bay N. Influence of workpiece surface topography on the mechanisms of liquid lubrication in strip drawing. Journal of tribology 2001; 123 (2): 290-294.

[7] Ahmed R, Sutcliffe MPF. An experimental investigation of surface pit evolution during cold-rolling or drawing of stainless steel strip. ASME Journal of Tribology 2001; 123(1): 1-7.

[8] Laugier M, Boman R, Legrand N, Ponthot J-P, Tornicelli M, Bech J, Carretta Y. Micro-plastohydrodynamic lubrication: a fundamental mechanism in cold rolling. Advanced Materials Research: Tribology in Manufacturing Processes \& Joining by Plastic Deformation 2014; 228-241.

[9] Challen JM, Oxley PLB. An explanation of the different regimes of friction and wear using asperity deformation models. Wear 1979; 53(2): 229 - 243.

[10] Reynolds O. On the theory of lubrication and its application to Mr. Beauchamp tower's experiments, including an experimental determination of the viscosity of olive oil. Philosophical Transactions of the Royal Society B 1886; 177: 157-234.

[11] Patir N, Cheng HS. Application of average flow model to lubrication between rough sliding surfaces. Journal of Lubrication Technology 1979; 101 (2): 220-229.

[12] Hu Y-K, Liu WK. An ALE hydrodynamic lubrication finite element method with application to strip rolling. International Journal for Numerical Methods in Engineering 1993; 36: 855-880.

[13] Boman R, Ponthot J-P. Finite element simulation of lubricated contact in rolling using the arbitrary Lagrangian-Eulerian formulation. Computer Methods in Applied Mechanics and Engineering 2004; 193 (39-41): 4323 - 4353.

[14] Yang T-S, Lo S-W. A finite element analysis of full film lubricated metal forming process. Tribology International 2004; 37(8): 591 - 598.

[15] Wilson WRD, Marsault NN. Partial hydrodynamic lubrication with large fractional contact areas. The American Society of Mechanical Engineers, Journal of Tribology 1998; 120(1): 16-20.

[16] Wilson WRD, Sheu S. Real area of contact and boundary friction in metal forming. International Journal of Mechanical Sciences 1988; 30(7): 475-489.

[17] Sutcliffe MPF. Surface asperity deformation in metal forming processes. International Journal of Mechanical Sciences 1988; 30(11): 847 - 868. 
[18] Sheu S, Wilson WRD. Mixed Lubrication of Strip Rolling. Tribology Transactions 1994; 37(3): 483493.

[19] Marsault N, Modélisation du régime de lubrification mixte en laminage à froid (in French). PhD thesis, Ecole nationale supérieure des mines de Paris, 1998 ; France.

[20] Qiu Z, Yuen W, Tieu A. Mixed-film lubrication theory and tension effect on metal rolling processus. Journal of Tribology 1998; 121: 908-915.

[21] Stephany A. Contribution à l'étude numérique de la lubrification en régime mixte en laminage à froid (in French). PhD thesis, University of Liège, 2008; Belgium.

[22] Carretta Y, Boman R, Stephany A, Legrand N, Laugier M, Ponthot J-P. METALUB - A Slab Method Software for the Numerical Simulation of Mixed Lubrication Regime in Cold Strip Rolling. Proceedings of the Institution of Mechanical Engineers - Part J - Journal of Engineering Tribology 2011; 225(9): 894-904.

[23] Hol J, Meinders VT, Geijselaers HJM, Van den Boogaard AH. Multi-scale friction modelling for sheet metal forming: The mixed lubrication regime. Tribology International 2015; 85: 10-25.

[24] Westeneng JD. Modelling of contact and friction in deep drawing processes. Ph.D. thesis, University of Twente, 2001; The Netherlands.

[25] Lo SW, Wilson WRD. A Theoretical Model of Micro-Pool Lubrication in Metal Forming. Journal of Tribology 1999; 121(4): 731-738.

[26] Sutcliffe MPF, Le HR, Ahmed R. Modeling of Micro-Pit Evolution in Rolling or Strip-Drawing, Journal of Tribology 2000; 123(4): 791-798.

[27] Shimizu I, Martins P, Bay N, Andreasen J, Bech J. Influences of lubricant pocket geometry and working conditions upon micro-lubrication mechanisms in upsetting and strip drawing. International Journal of Surface Science and Engineering 2010; 4(1): 42-54.

[28] Hubert C, Bay N, Christiansen P, Deltombe R, Dubar L, Dubar M, Dubois A. Numerical simulation of lubrication mechanisms at mesoscopic scale. AIP conference proceedings 2011; 1353(1): 17291734.

[29] Dubar L, Hubert C, Christiansen P, Bay N, Dubois A. Analysis of fluid lubrication mechanisms in metal forming at mesoscopic scale, CIRP Annals - Manufacturing Technology 2012; 61(1): 271-274.

[30] METAFOR. A large strain finite element software. A\&M-MN2L, University of Liège, http://metafor.Itas.ulg.ac.be/. Accessed July 2016.

[31] Neumann M, Tiyyagura SR, Wall WA, Ramm E. Robustness and efficiency aspects for computational fluid-structure interaction. Computational Science and High Performance Computing II 2006; 91: 99-114.

[32] Degroote J, Bathe KJ and Vierendeels J. Performance of a new partitioned procedure versus a monolithic procedure in fluid-structure interaction. Computers \& Structures 2009; 87(11-12): 793801. 
[33] Ponthot JP. Unified stress update algorithms for the numerical simulation of large deformation elasto-plastic and elasto-viscoplastic processes. International Journal of Plasticity 2002; 18(1): 91126.

[34] Donéa J, Huerta A, Ponthot J-P, Rodriguez-Ferran A Encyclopedia of computational mechanics. In chap. 14: Arbitrary Lagrangian-Eulerian Methods. Vol. 1, Stein E, de Borst R, Hughes T J R (eds). John Wiley \& Sons, 2004; 413-437, DOI: 10.1002/0470091355.ecm009

[35] Boman R, Ponthot J-P. Efficient ALE mesh management for 3D quasi-Eulerian problems, International Journal for Numerical Methods in Engineering, 2012; 92(10): 857-890.

[36] Chung J, Hulbert JM. A time integration algorithms for structural dynamics with improved numerical dissipations: the generalized- $\alpha$ method. Journal of Applied Mechanics 1993; 60: 371-375.

[37] Ghia U, Ghia KN, Shin CT. High-resolutions for incompressible flows using Navier-Stokes equations and a multigrid method. Journal of Computational Physics 1982; 48: 387-411.

[38] Schreiber R, Keller HB. Driven cavity flows by efficient numerical techniques. Journal of Computational Physics 1983; 49(2): 310-333.

[39] Peng Y-F, Shiau Y-H, Hwang R. Transition in a 2-D lid-driven cavity flow. Computers and Fluids 2003; 32(3): 337-352.

[40] Sahin M, Owens RG. A novel fully-implicit finite volume method applied to the lid-driven cavity problem. Parts I and II. International Journal for Numerical Methods in Fluids 2003; 42(1): 57-88.

[41] Bruneau C-H, Saad M. The 2D lid-driven cavity problem revisited. Computers and Fluids 2006; 35(3): $326-348$.

[42] FrêneJ. Butées et paliers hydrodynamiques. Techniques de l'ingénieur, 1995.

[43] Williams JA. Engineering Tribology. Oxford Science Publication 1998.

[44] Weller HG, Tabor G, Jasak H, Fureby C. A tensorial approach to computational continuum mechanics using object-oriented techniques. Computers in Physics 1998; 12(6): 620-631.

[45] Zienkiewicz O, Taylor R. The Finite Element Method. Volume 3: Fluid Dynamics. ButterworthHeinemann 2005.

[46] Glaser S, Armero F. On the formulation of enhanced strain finite elements in finite deformations. Engineering Computations: International Journal for Computer-Aided Engineering 1997; 14(7): 759-791.

[47] Simo JC, Armero F, Taylor RL. Improved versions of assumed enhanced strain trilinear elements for 3D finite deformation problems. Computer Methods in Applied Mechanics and Engineering 1993; 110(3-4): 359-386.

[48] Bui QV, Papeleux L, Ponthot J-P. Numerical simulation of springback using enhanced assumed strain elements. Journal of Materials Processing Technology 2004; 153-154: 314-318. 
[49] Adam L, Ponthot J-P. Thermomechanical modeling of metals at finite strains : First and mixed order finite elements. International Journal of Solids and Structures 2005; 42: 5615-5655.

[50] Souli M, Benson DJ. Arbitrary Lagrangian Eulerian and Fluid-Structure Interaction: Numerical Simulation, Wiley 2010.

[51] Wautelet G, Ponthot J-P. The influence of equivalent contact area computation in extended node to surface contact elements, Key Engineering Materials 2014; 618: 1-22.

[52] Wautelet G, Papeleux L, Ponthot J-P. The Influence of Equivalent Contact Area Computation in 3D Extended Node to Surface Contact Elements. Key Engineering Materials (Trans Tech Publications) 2016; 681: 19-46.

[53] Sarrate J, Huerta A. Efficient unstructured quadrilateral mesh generation. International Journal of Numerical Methods in Engineering 2000; 49: 1327-1350.

[54] Ponthot J-P. Traitement unifié de la Mécanique des Milieux Continus solides en grandes transformations par la méthode des éléments finis. Ph.D. Thesis (in French), University of Liège, Liège, 1995; Belgium.

[55] Giuliani S. An algorithm for continuous rezoning of the hydrodynamic grid in Arbitrary Lagrangian-Eulerian computer codes. Nuclear Engineering and Design 1982 ; 72(2): 205-212.

[56] Dwyer-Joyce RS, Drinkwater BW and Donohoe CJ. The measurement of lubricant-film thickness using ultrasound. Proceedings of the Royal Society A 2003; 459: 957-976.

[57] Dwyer-Joyce RS, Harper $P$ and Drinkwater BW. A Method for the Measurement of Hydrodynamic Oil Films Using Ultrasonic Reflection. Tribology Letters 2004; 17(2): 337-348.

[58] Reddyhoff T, Dwyer-Joyce RS, Harper P. A new approach for the measurement of film thickness in liquid face seals. Tribology Transactions 2008; 51 (2): 140-149.

[59] Hunter A, Dwyer-Joyce RS, Harper P. Calibration and validation of ultrasonic reflection methods for thin-film measurement in tribology. Measurement Science and Technology 2012; 23(10).

This article is protected by copyright. All rights reserved. 